\title{
Mover affects a subpool of primed synaptic vesicles in the mouse calyx of Held
}

\author{
Dissertation \\ for the award of the degree \\ "Doctor rerum naturalium" \\ of the Georg-August-Universität Göttingen
}

within the doctoral program Sensory and Motor Neuroscience of the Georg-August University School of Science (GAUSS)

\author{
submitted by \\ Ermis Pofantis \\ from Athens, Greece
}

Göttingen, 2019 

Prof. Dr. Thomas Dresbach

Department of Anatomy and Embryology,

University of Göttingen, Göttingen

Prof. Dr. Nils Brose

Department of Molecular Neurobiology, Max Planck

Institute for Experimental Medicine, Göttingen

Prof. Dr. Tobias Moser

Institute for Auditory Neuroscience and InnerEarLab, University Medical Center, Göttingen

MEMBERS OF THE EXAMINATION BOARD

Referee: Prof. Dr. Dr. h.c. Erwin Neher

Department of Membrane Biophysics, Max Planck

Institute for Biophysical Chemistry, Göttingen

2nd Referee: Camin Dean, Ph.D.

European Neuroscience Institute, Göttingen

3rd Referee: Prof. Dr. Luis A. Pardo

Department of Molecular Biology of Neuronal Signals, Max Planck Institute for Experimental Medicine, Göttingen 

To my family

\& to my teachers 



\section{Table of Contents}

\section{ABSTRACT}

II GLOSSARY OF SYMBOLS AND ABBREVIATIONS

I

I7 INTRODUCTION

I7 The neuron \& the synapse

I8 Neurotransmitter receptors

2I The presynapse

28 The function of the sv release machinery in detail

28 Release probability

28 Synaptic vesicle functional pools

30 Short-term plasticity

33 Multiple sv priming states

37 The calyx of Held

40 Mover

42 Aim of this study

2

43 MATERIALS AND METHODS

43 Animals

43 Slice preparation

46 Electrophysiology

47 Data analysis and statistics

3

49 RESULTS

5I Mover ko does not affect basic synaptic transmission

53 Mover ko does not affect evoked release 
54 Initial release probability is decreased in the mover kO

54 Paired-pulse ratio is increased in the ko synapses

56 High-frequency stimulation-train time constant but not extent of depression is increased in the ko

57 Readily-releasable pool size

59 Recovery from synaptic depression is unaffected

6o The superprimed sv pool is affected in mover ko

66 Lower release probability of the ko tight-state component

66 Non-negative tensor factorization theory

77 NTF results

\section{4 \\ 85 DISCUSSION \\ 85 The primary finding: Mover is increasing the initial release probability of tight-state synaptic vesicles}

85 The premise of the study: reasons to study the role of Mover

86 A brief overview of the results of this study: deriving the major conclusion

89 Methodological considerations: pool estimation and blind-source separation

9I Mover and Bassoon: is their interaction involved in the observed phenotype?

92 Mover's function in other synapses of the brain

93 Knockdown of Mover in rat versus knockout in the mouse

97 Additional points to ponder: LS-to-TS transition versus Ts pool size

97 Future endeavors

IOI REFERENCES

II5 ACKNOWLEDGEMENTS 


\section{Abstract}

Neuronal communication is a complex process; synapses must be formed, neurotransmitter has to be released at precise time points and it has to be "sensed" by the receiving end of a synapse with an equal accuracy. In this highly coordinated ballet of proteins any change may result in disharmony and eventually in pathology. Therefore, any new addition during the course of evolution must be fulfilling a specific purpose.

A relatively new protein in the evolutionarily highly-conserved presynaptic apparatus, since it is vertebrate specific, is Mover. It is attached to synaptic vesicles and interacts with Calmodulin and Bassoon, another vertebrate-specific active zone protein. Mover's expression levels vary throughout the brain, suggesting a modulatory function at the operation of the synapses.

Here, I aimed to elucidate Mover's role in synaptic transmission in the calyx of Held, a central glutamatergic synapse, using a Mover knockout (ко) mice. To this end, I recorded spontaneous and evoked excitatory postsynaptic currents (EPSCS) from brainstem slices using a whole-cell patch clamp configuration.

In the KO evoked EPSCS were slightly smaller, and took longer to reach the same steady-state levels as the wild-type upon high frequency stimulation. Applying a blind-source separation technique termed non-negative tensor factorization allowed me to distinguish between different subpools of vesicles. This analysis gave rise to a model in which the absence of Mover reduces the release probability of a subpool of vesicles, termed "tight-state" vesicles -referring to the conformation of the SNARE complex and its associated proteins. Additionally, the size of this pool is significantly increased, indicating a compensatory mechanism. In contrast, the loose-state synaptic vesicles, the functional precursors of the tight-state ones, are unaffected by the absence of Mover.

These findings suggest that Mover modulates the initial release probability, by specifically influencing the subpool of these tightstate vesicles. 

Glossary of Symbols and Abbreviations

\section{PRINCIPAL SYMBOLS}

A Ampere

C Celsius

$\mathrm{Hz}$ Herz

1 litre

M mol

$n$ number of samples

$\mathrm{O} \mathrm{Ohm}$

Osm osmol

$p$ singificance level

$p_{n} \quad$ release probability of normally primed synaptic vesicles

$p_{\text {occ }}$ probability that a release site will be occupied by a synaptic vesicle

$p_{s} \quad$ release probability of superprimed synaptic vesicles

$p_{\text {succ }}$ probability that a synaptic vesicle occupying a release site will be succesfully released

$p_{T S} \quad$ tight-state release probability

$q$ quantal size of a synaptic vesicle

$t$ size of the difference relative to the variation in the sample data

V Volt

$\tau$ time constant

$\nabla$ gradient operator

\section{AbBreviations}

ACSF Artificial Cerebrospinal Fluid

AICc Akaike's Information Criteria 


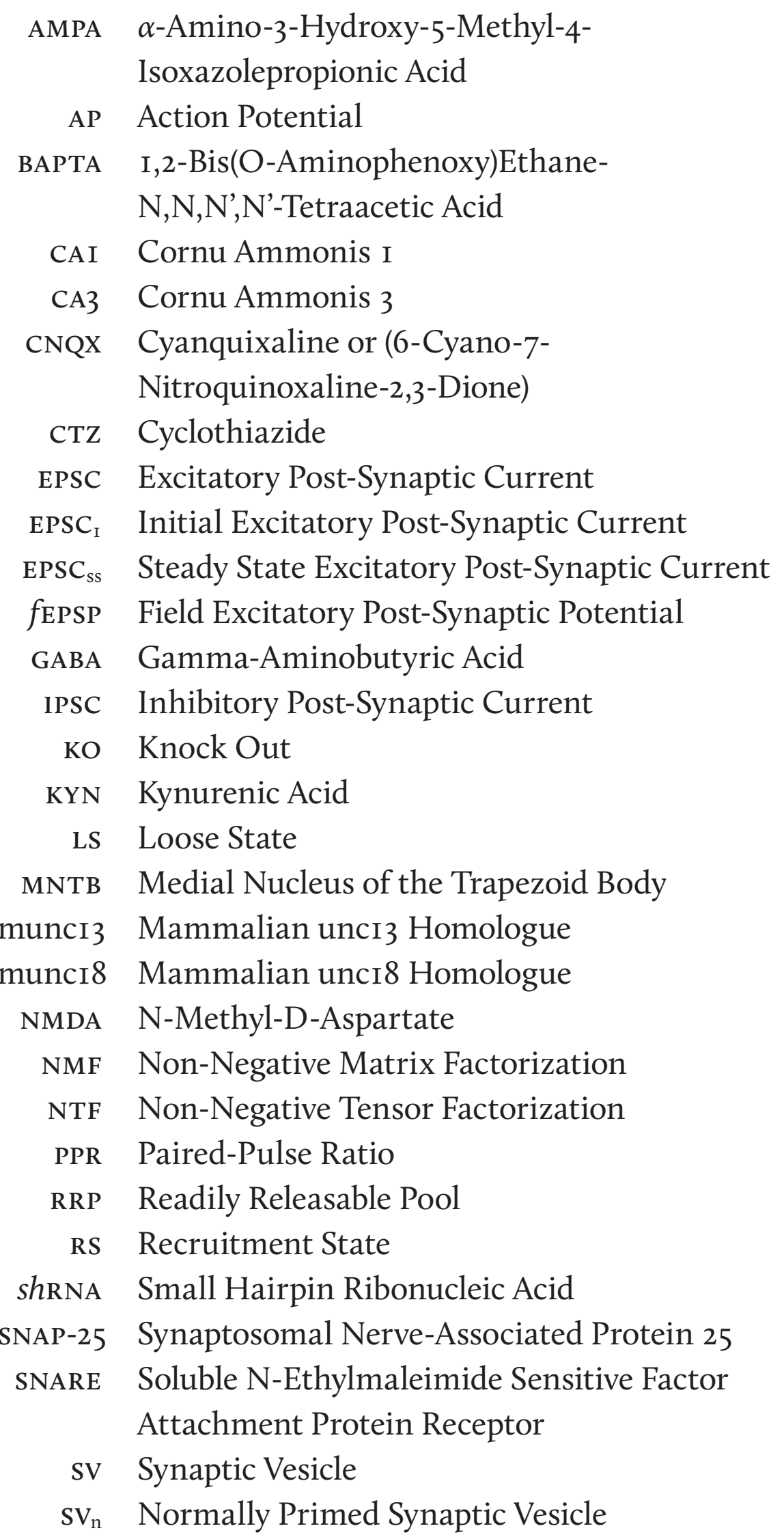


$\mathrm{SV}_{\mathrm{s}} \quad$ Superprimed Synaptic Vesicle

syt-I synaptotagmin I

Ts Tight State

TTX Tetrodotoxin

VAMP Vesicle-Associated Membrane Protein

wT Wild Type 


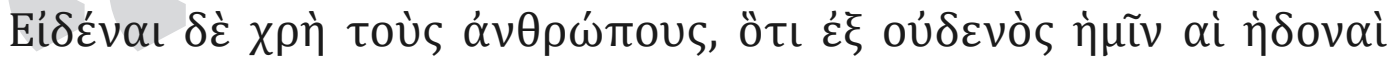

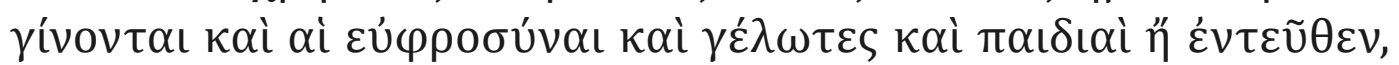

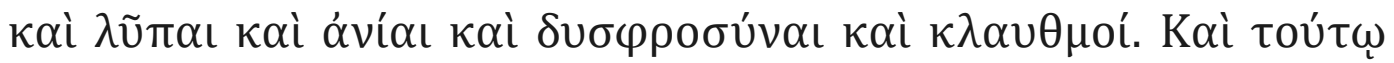

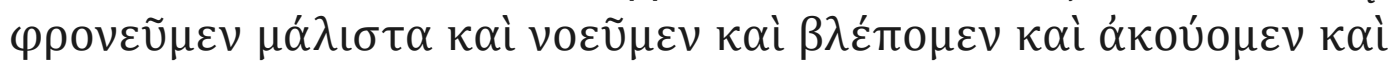

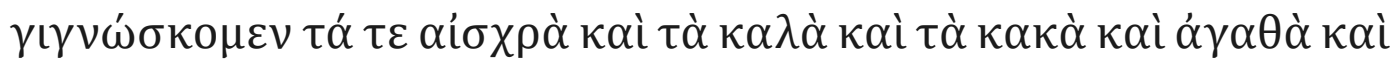
$\grave{\eta} \delta \varepsilon \dot{\alpha} \alpha \alpha \grave{~} \alpha \dot{\eta} \delta \dot{\alpha} \alpha$.

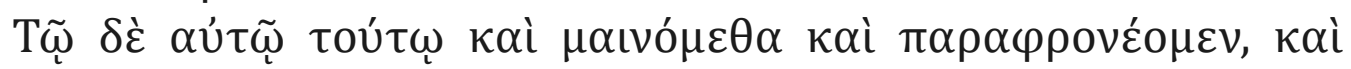

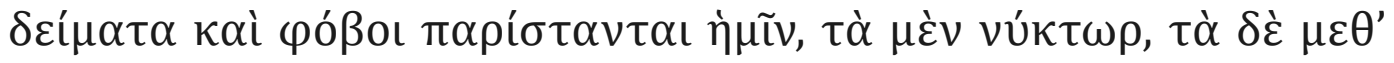

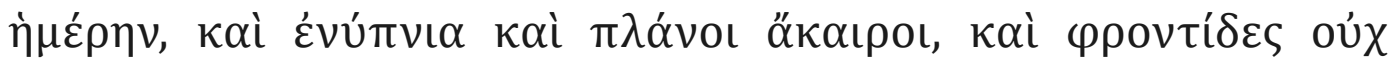

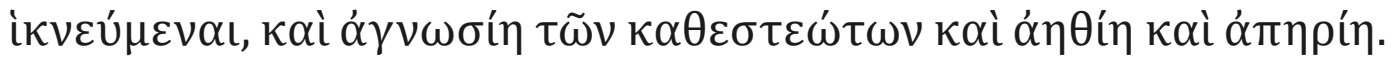

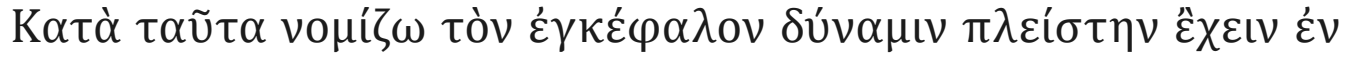
$\tau \tilde{\omega} \alpha \dot{\alpha} v \theta \rho \omega \dot{\omega} \pi \omega$.

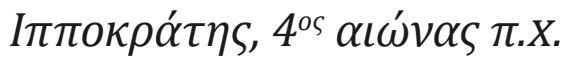


It must be known that from the brain come the pleasures, the joys, the laughters and the games; also the sorrows, the pains, the discontents and the wailings. And with this, we think, we understand, we see, we hear and we recognize the ugly and the beautiful, the bad and the good.

It is again from there that we become mad and delirious, and that fears and terrors besiege us, either at night, or after the coming of the day, dreams, untimely errancies, worries without motives, ignorance of the present, unethicalities, inexperience.

For these reasons I regard the brain as exercising the greatest power in man.

Hippocrates, $4^{\text {th }}$ century B.C. 



\section{Introduction}

Imagine you are at the seaside during sunset. The sky has a fiery orange color that extends all the way to a dark blue. The only sound comes from the sea, while gently touching the shoreline. A human would most likely find this situation pleasant and relaxing, yet from a physics standpoint it's nothing but a bombardment of numerous electromagnetic frequencies and pressure oscillations in the air. Still, all these are perceived by the brain in such a way that it produces a meaningful result.

This happens due to the integration of the work of billions of microscopic calculators, the neurons, which "talk" to each other through highly complex and specialized points of communication, the synapses. It is the aim of this study to contribute to the knowledge on how synapses operate and to identify how a part of this machinery is influencing their function. This part is a protein called Mover.

\section{I.I THE NEURON \& THE SYNAPSE}

The cells of the nervous system can be broadly divided into neurons and glia. Although there are about equal numbers of neuronal and glial cells in the adult human brain (von Bartheld et al., 20I6), only neurons are capable of transmitting electrical signals at long distances, at least most of them. Glia, on the other hand are responsible for supporting the function of neurons, by insulating, nurturing them and even by repairing damage in the developing brain. While glia's function is equally important in a normally functioning brain, this study revolves around synaptic transmission; therefore the focus will be on neurons.

Neurons are cells that are specialized both for intracellular signaling but also for intercellular communication. Intracellularly, they transfer electrical signals at long distances. Intercellularly they communicate with other cell types, including other neurons, by means of synapses. The most obvious feature of their specialization for com- 
munication is their extensive branching, with the dendrites and the axon being the most prominent branches.

The term dendrite comes from the Greek word for "tree", reflecting their similar branching pattern. Usually, dendrites emanate from the cell body and then they elaborately arborize as their typical function is to receive incoming signals from other neurons. An axon, on the other hand, extends to longer distances and conveys an electrical signal, the action potential, up to its very end where it can transfer information to other cells, through synapses.

The action potential is an all-or-nothing change in the polarity of the cell's membrane potential that actively propagates from its beginning, the axon hillock, until the presynaptic terminals. An action potential is initiated by an influx of $\mathrm{Na}^{+}$ions, which depolarize the cell membrane. It is terminated through an efflux of $\mathrm{K}^{+}$ions that are restoring the original charge separation and repolarize the membrane to its resting potential.

An action potential reaching the presynaptic terminal can trigger a cascade of events that result in the transfer of information from one cell to the other through synaptic transmission. The majority of synaptic transmission occurs through chemical synapses, where the presynaptic terminal of an axon releases into the extracellular space neurotransmitter molecules; the neurotransmitter is then triggering the flow of electrical current in the postsynaptic neuron through receptors on membrane specializations that are usually situated on dendrites, or more rarely on a cell's soma or even on another axon.

\section{I.I.I Neurotransmitter receptors}

The neurotransmitter receptors are proteins embedded in the postsynaptic density that can bind neurotransmitter molecules. This binding triggers a series of conformational changes so that the end result is a change in the functioning of this protein. Neurotransmitter receptors are classified into two broad categories: ionotropic and metabotropic. They can also be divided into excitatory and inhibitory, reflecting the changes they induce on the postsynaptic cell.

The ionotropic receptors form an ion channel, as they contain a membrane-spanning domain. Thus, they create a pore in the cell membrane through which they allow ions to pass. They combine 
molecule-binding and ion-channel functions into one molecular unit and usually when a neurotransmitter molecule is not bound on them, they are closed. When a neurotransmitter is attached to a specific site on them, they change their conformation, permitting the flow of certain categories of ions, depending on the receptor's composition.

However, the direction that these ions flow depends on the equilibrium potential for each ion. If a receptor is permeable to $\mathrm{Na}^{+}$ions, then the net result when this receptor opens will be the depolarization of the cell because there will be an influx of $\mathrm{Na}^{+}$ions. If, on the other hand, the receptor is allowing $\mathrm{Cl}^{-}$ions to pass through, then the cell will be hyperpolarized. This happens because the equilibrium potential of $\mathrm{Cl}^{-}$is usually lower than the resting membrane potential, thus the opening of these channels cause an influx of chloride.

The major excitatory neurotransmitter in the brain is the amino acid L-glutamate and the main broad categories of ionotropic glutamate receptors are AMPA, NMDA and kainate. AMPA and NMDA were named after their synthetic agonists whereas kainate receptor cloning followed the discovery of its agonist, kainic acid, in a seaweed known for its effectiveness at killing intestinal worms. The name "kainic" comes from the Japanese word kaininso, meaning "the ghost of the sea" (Hammond, 2015).

AMPA-gated channels are composed of four subunits arranged around a central pore. There are four different types of subunits, termed GluAi to GluA4, and their different combinations confer unique attributes to the channel. AMPA channels are permeable to $\mathrm{Na}^{+}$and $\mathrm{K}^{+}$ions, but they are mostly impermeable to $\mathrm{Ca}^{2+}$. Therefore, when they are activated at a normal membrane potential, they are rapidly depolarizing the membrane because more $\mathrm{Na}^{+}$is entering the cell than the $\mathrm{K}^{+}$that is leaving it.

The excitatory postsynaptic current (EPSC) produced by an AMPA receptor usually is larger than that produced by an NMDA receptor. This makes the AMPA receptors the primary mediators of excitatory synaptic transmission in the brain. In contrast, the NMDA receptors mediate EPSCs that are longer in duration.

NMDA channels are also comprised from 4 subunits. There are five different types of subunits that are divided into two groups; the first group has the single GluNi subunit and the second group the four 
GluN2A-D subunits. Each receptor is composed by two GluNi subunits and two of the GluN2 subunits.

One of the most special properties of the NMDA receptors is the fact that they allow the entry of $\mathrm{Ca}^{2+}$ into the cell, along with $\mathrm{Na}^{+}$and $\mathrm{K}^{+}$. This allows the buildup of intracellular $\mathrm{Ca}^{2+}$ which then acts as a second messenger for numerous signaling pathways. Another major difference is that in order to be activated they require a depolarized membrane potential. This is because at resting membrane potentials (around $-65 \mathrm{mV}$ ) the channel's pore is blocked by $\mathrm{Mg}^{2+}$. So even if glutamate binds to the receptor, the entry of $\mathrm{Ca}^{2+}$ and $\mathrm{Na}^{+}$is still blocked. When the membrane is depolarized, usually through the activation of AMPA receptors, then the $\mathrm{Mg}^{2+}$ ion is expelled through electrostatic repulsion and $\mathrm{Ca}^{2+}, \mathrm{K}^{+}$and $\mathrm{Na}^{+}$can flow freely. Due to this double prerequisite, the presence of glutamate and a depolarized cell, the NMDA receptor acts as a "coincidence detector", detecting the joint activation of the presynaptic and the postsynaptic cell.

Kainate receptors share many properties with AMPA receptors, as they are made up from different combinations of five subunits: GluKI-5. Their physiological role is much less investigated; sometimes they are found in the presynaptic terminals serving as a feedback mechanism and sometimes they are situated postsynaptically. The EPSC they produce has a fast rise time, it is usually not reaching the amplitude levels of an AMPA EPSC and it has a much slower decay time.

Metabotropic excitatory receptors do not have an ion channel as part of their structure; instead, they are relying on the activation of intermediary proteins, called G-proteins, to affect ion channels. Upon binding of a neurotransmitter on the extracellular part of the receptor, a G-protein is released from the intracellular side; this $\mathrm{G}$-protein is then activating effector proteins, such as enzymes, which are producing intracellular messenger molecules that in turn activate ion channels.

Thus, the ionotropic receptors are geared towards a faster response to the release of neurotransmitter whereas the metabotropic receptors are acting more slowly, at the scale of less than a millisecond up to minutes, hours or even days. The combined action of these two types of receptors can amplify a presynaptic signal many times over and they are essential for inducing long-term changes in the synapse. 
The two major inhibitory neurotransmitters in the central nervous system are $\gamma$-aminobutyric acid (GABA) and glycine. GABAergic synapses have two types of postsynaptic receptors: $\mathrm{GABA}_{\mathrm{A}}$ which are ionotropic receptors and $\mathrm{GABA}_{\mathrm{B}}$ which are metabotropic. $\mathrm{GABA}_{\mathrm{A}}$ receptors are pentamers; they are comprised of five subunits, but the total number of different subunits available is I9, allowing these channels to exhibit a great variety across neuronal types.

$\mathrm{GABA}_{\mathrm{A}}$ channels are permeated by anions, meaning that when they are activated they allow $\mathrm{Cl}^{-}$to flow through them. Due to the fact that the reversal potential for $\mathrm{Cl}^{-}$is slightly more negative $(-70 \mathrm{mV})$ than the typical resting membrane potential $(-65 \mathrm{mV})$, when these receptors open the influx of $\mathrm{Cl}^{-}$causes the postsynaptic cell to be hyperpolarized.

The metabotropic $\mathrm{GABA}_{\mathrm{B}}$ receptors exert their action through a second-messenger pathway that ultimately often activates a $\mathrm{K}^{+}$channel. Due to the fact that the reversal potential for $\mathrm{K}^{+}$is at $-80 \mathrm{mV}$, the opening of this channel causes a hyperpolarization of the membrane. $\mathrm{GABA}_{\mathrm{B}}$ inhibitory postsynaptic currents (IPSCS) turn on more slowly but persist for a longer time than the IPSCs induced by $\mathrm{GABA}_{\mathrm{A}}$ receptors.

Glycine receptors are also ionotropic inhibitory channels, consisting of five subunits; four types of $\alpha$ and one type of $\beta$ subunit. Glycine channels are also permeated by $\mathrm{Cl}^{-}$, with a slightly greater conductance $(46 \mathrm{pS})$ than the $\mathrm{GABA}_{\mathrm{A}}$ receptors (30 pS).

\section{I.I.2 The presynapse}

\section{Neurotransmitters}

The life cycle of a chemical neurotransmitter can be divided into four steps, which begin with its synthesis and storage, its release into the synaptic cleft, continue with the interaction of the transmitter with postsynaptic receptors and end with its removal from the synaptic cleft by means of uptake and recycling by the presynaptic terminal. We have already discussed the third step which involves the postsynapse. In this section, I will focus on the three other steps which occur at the presynaptic terminal. 
First, it needs to be defined what is considered as a neurotransmitter. As a general rule, a neurotransmitter is a substance that is released by a neuron in order to transfer information to a specific target. Information transfer is defined as the induction of specific changes to the target, which can be another neuron or an organ, such as a muscle.

A more specific definition of a neurotransmitter would be the following four criteria: (I) a neurotransmitter must be synthesized in the presynaptic neuron. (2) It must be present in the presynaptic terminal and its release must induce changes in the postsynaptic target. (3) It must induce the same changes in the postsynaptic targets even when it is administered exogenously. (4) It must be actively removed from the synaptic cleft.

In the nervous system, there are two types of neurotransmitters; small-molecule transmitters, which tend to mediate rapid postsynaptic actions and neuroactive peptides which usually modulate slower functions.

Small-molecule transmitters are usually synthesized locally in the presynaptic terminal. The precursor molecules for their synthesis are usually taken into the terminal by specific transporter proteins found on the plasma membrane of the terminal. Then, specific enzymes found in the terminal are synthesizing the neurotransmitters, which are subsequently loaded inside synaptic vesicles (svs).

Glutamate, the most frequently used excitatory neurotransmitter in the central nervous system, is synthesized from $\alpha$-ketoglutarate, which is in turn produced during the tricarboxylic acid (Krebs) cycle. Then, glutamate is loaded into the synaptic vesicles by vesicular glutamate transporters (VGLUTs). After its release into the synaptic cleft, glutamate is taken up by both neurons and glia. In astrocytes the enzyme glutamine synthase is converting it to glutamine. Then it is transported back to neurons where it is hydrolyzed to glutamate by the enzyme phosphate-activated glutaminase.

The amino acid GABA, is the major neurotransmitter used by inhibitory neurons and interneurons in the brain. Its precursor molecule is glutamate and the enzyme glutamic acid decarboxylase is catalyzing the reaction along with a co-factor, pyridoxal phosphate.

The other main amino-acid which is used by inhibitory neurons is glycine. Its spread among the central nervous system is more re- 
stricted, with half of the inhibitory neurons in the spinal cord using it and most other inhibitory synapses using GABA. It is synthesized from serine, via the enzyme serine hydroxymethyltransferase. Once released into the synaptic cleft, it is removed by glycine transporters in the plasma membrane. Both glycine and GABA are packed into synaptic vesicles by a vesicular inhibitory amino-acid transporter (VGAT).

\section{Synaptic vesicle cycling}

Neurotransmitters do not circulate freely inside the presynaptic terminal; instead they are packed into synaptic vesicles. These svs are then docked at the presynaptic membrane, become fusion-capable (primed) and then they fuse with the presynaptic membrane, releasing their contents into the synaptic cleft. This fusion of svs causes new membrane to be added to the plasma membrane, so this extra membrane is quickly removed and used for the production of new svs.

Fast, synchronous neurotransmitter release is triggered by $\mathrm{Ca}^{2+}$ entry into the presynaptic active zone through $\mathrm{Ca}_{v} 2$ channels (Dunlap et al., 1995). These are voltage-gated $\mathrm{Ca}^{2+}$ channels that require strong depolarization to be activated and they are the major sources of $\mathrm{Ca}^{2+}$ entry into the presynapse. $\mathrm{Ca}_{v}$ 2.I channels are found in fast synapses in the central nervous system, like the calyx of Held, as well as in the mammalian neuromuscular junction. The other subtype, $\mathrm{Ca}_{v} 2.2$ channels are predominantly located in the peripheral nervous system (Olivera et al., I994). A third, less studied subtype are the $\mathrm{Ca}_{v} 2.3$ channels which are thought to contribute to synaptic transmission in the central nervous system (Gasparini et al., 200I).

When a $\mathrm{Ca}^{2+}$ channel opens, an elevated calcium concentration is quickly created and it surrounds the channel inside the cell; this gradient of increased $\mathrm{Ca}^{2+}$ is called a calcium nanodomain. When multiple nearby $\mathrm{Ca}^{2+}$ channels open for a prolonged period of time the individual nanodomains collapse into a larger calcium microdomain.

$\mathrm{Ca}^{2+}$ entering the presynaptic compartment acts through the SNARE complex (soluble N-ethylmaleimide-sensitive fusion protein), a crucial part of the vesicle fusion machinery. The SNARE-complex proteins are universally mediating membrane fusion, from yeast to humans. A series of studies on the molecular targets of the bacte- 
rial tetanus and botulinum toxins revealed the essential role of the SNARE complex in synaptic vesicle fusion (Jahn and Niemann, I994; Montecucco and Schiavo, 1993).

One of the SNARE proteins, synaptobrevin, is attached on the membrane of synaptic vesicles and it forms a complex with the active zone SNARE proteins syntaxin and SNAP-25. All these proteins can bind together bringing the synaptic vesicle close to the cell membrane. In essence, the SNARE motif of synaptobrevin forms a tight complex with the SNARE motifs of syntaxin and SNAP-25 (SNAP-25 has two SNARE motifs, whereas the other two proteins have one motif each). This results in a progressive zippering of the four-helical SNARE complex that brings the fusing membranes so close together that their hydrophilic surfaces are destabilized.

Although the membranes of the synaptic vesicle and of the active zone are brought close together by the zippering of the SNARE complex, this event is not adequate to promote their fusion. In vitro, the overall time for membrane fusion mediated by SNARES can take tens of miliseconds (Karatekin et al., 20Io) up to hours (Weber et al., I998) compared with the sub-millisecond range of synaptic vesicle fusion in vivo. An additional protein which comes into play is Munci8 (mammalian unci8 homologue), which binds to syntaxin during trafficking of these proteins at the active zone. Munci8 is not essential for neuronal assembly during development but it's required for the maintenance of synaptic connectivity; when the protein is absent, there is no vesicle docking in the active zones and no synaptic events are observed. This lack of synaptic activity eventually results in neuronal apoptosis and widespread degeneration (Verhage et al., 2000; Voets et al., 200I).

Similarly, when members of the Unc-I3/Munci3 protein family are genetically inactivated from central synapses, a complete silencing of synaptic transmission ensues (Augustin et al., I999). Further studies revealed that this silencing is due to a total loss of primed synaptic vesicles and an inability for Sv fusion (Varoqueaux et al., 2002). However, no reduction in the number of primed vesicles was observed in ultrastructural analysis using chemically fixed specimens (Augustin et al., 1999).

The term "priming" refers to the assignment of fusion competence to synaptic vesicles that are already docked to the active zone. The 
ways that priming can be achieved will be discussed in detail, in this chapter.

Using techniques such as high-pressure freezing and freeze substitution electron microscopy that are capable of near-instantaneous fixation, it was found that the number of svs that are in direct contact with the plasma membrane are significantly reduced in C. elegans (Weimer et al., 2006) and in mice (Siksou et al., 2009). In particular, there were almost no svs within $2 \mathrm{~nm}$ from the active zone in Munci3-deficient organotypic hippocampal slice cultures and very few within 2-4 $\mathrm{nm}$. In contrast, svs 5-20 $\mathrm{nm}$ away from the active zone were significantly more numerous (Imig et al., 20I4). The loss of svs within $2 \mathrm{~nm}$ of the active zone indicated an absence of primed vesicles.

Interestingly, when Munci8-I or Munci3-I were absent from hippocampal synapses, a "de-priming" effect was observed; svs were primed after a high-frequency stimulation, however they would fall back to a de-primed state after a few seconds of inactivity. In detail, synapses deficient in Munci8-I or Munci3-I showed a severely reduced basal transmission. However, after a Ioo-stimuli $40-\mathrm{Hz}$ train EPSCS were strongly potentiated even until $50 \mathrm{~s}$ afterwards (He et al., 2017).

Thus, the essential fusion machinery in the cell is comprised of SNARE, Munci8 and Munci3 homologue proteins. Additional proteins can then modulate it according to the needs of specific fusion reactions.

One such modulator is tomosyn. Tomosyn, which was discovered in Yoshimi Takai's lab in 1998 and named "friend of syntaxin" (tomo in Japanese), is a binding protein of syntaxin. By blocking SNARE-complex formation in a $\mathrm{Ca}^{2+}$-dependent manner it inhibits $\mathrm{Ca}^{2+}$-mediated synaptic vesicle fusion (Fujita et al., I998). Tomosyn can exist in two conformational states. In one state, the tail domain binds to the WD4O N-terminal repeats and thus the C-terminal vAMP-like domain can inhibit the SNARE complex formation (Yamamoto et al., 2009). In the other state, the tail domain binds to the c-terminal vamp-like domain and subsequently the vAMP-like domain's inhibitory effect is diminished. Additionally, tomosyn using its N-terminal WD40 repeats can directly bind to synaptotagmin-I, a $\mathrm{Ca}^{2+}$ sensor protein. Therefore, synaptotagmin-I is impaired to act on SNARE-mediated 
vesicle fusion and tomosyn can target SNAREs more efficiently, thus having an improved inhibitory effect on $\mathrm{Ca}^{2+}$-mediated transmitter release (Yamamoto et al., 20I0).

A second modulator of the fusion machinery is a small cytoplasmic protein called complexin. It has been suggested that complexin has at least two independent roles: it prevents membrane fusion by clamping the trans-SNARE complex and thus preventing its full zippering (Giraudo et al., 2006) and it regulates synchronous $\mathrm{Ca}^{2+}$-dependent exocytosis. The SNARE-binding domain of complexin is required for both of these functions.

The clamping role of complexin is mediated through its central and $\mathrm{N}$-terminal accessory helices (Giraudo et al., 2009). However, when a $\mathrm{Ca}^{2+}$ signal arrives, the complexin clamp can be relieved by the binding of synaptotagmin-I in complexin's c-terminal region through a competitive or cooperative fashion (Tang et al., 2006). Thus, fast $\mathrm{Ca}^{2+}$-induced neurotransmitter release is promoted.

A separate line of evidence indicates that mice lacking both complexins have a dramatically reduced fast synchronous $\mathrm{Ca}^{2+}$-triggered release (Maximov et al., 2009; Reim et al., 200I). This function of complexin was corroborated in neuronal cultures obtained from conditional knockout mice; spontaneous, synchronous, asynchronous as well as delayed synaptic vesicle fusion was attenuated. Additionally, no effect was observed on vesicle priming indicating that complexins may be dispensable for "fusion clamping" (Lopez-Murcia et al., 20I9).

The final step in neurotransmitter exocytosis is the transduction of the $\mathrm{Ca}^{2+}$ signal to the primed fusion machinery. This is mediated through a family of proteins called synaptotagmins. Synaptotagmins are transmembrane proteins that can bind to phosphlipid membranes. In addition, they have two $\mathrm{C}_{2}$ cytoplasmic domains that can bind $\mathrm{Ca}^{2+}$ and, in the case of synaptotagmin-I, can bind to syntaxin-I and SNARE complexes. Synaptotagmin-2 is thought to be the fastest one, as it is found in sound localization neurons which exhibit very fast synaptic responses. Synaptotagmin-I is the $\mathrm{Ca}^{2+}$-sensor for fast and synchronous transmitter release in response to an action potential (AP) (Brose et al., I992). Syt-I also supports priming of sVs, upon their docking at the plasma membrane (Wang et al., 20II). In 
contrast, synaptotagmin-9 is the slowest and it is localized in limbic system neurons (Xu et al., 2007).

One of the most interesting functions of synaptotagmin-I is its ability to synchronize the fusion of svs upon the arrival of an AP. In particular, transmitter release in syt-I-deficient synapses is completely desynchronized (Geppert et al., I994). However in syt-I-mutant synapses that also display an asynchronous first response, the phenotype is rescued when the first AP is closely followed by another at IO-50 ms; the second AP is markedly more synchronous and this effect is $\mathrm{Ca}^{2+}$-dependent (Chang et al., 20I8). Therefore, syt-I seems to be also supporting tight sv-attachment at the plasma membrane.

An additional modulator of synaptic activity during high-frequency stimulation is the $\mathrm{Ca}^{2+}$-influx-dependent facilitation and inhibition of presynaptic $\mathrm{Ca}_{v} 2$. I channels. As mentioned earlier, $\mathrm{Ca}_{\mathrm{v}} 2$ channels are the major sources of $\mathrm{Ca}^{2+}$ entering the presynapse upon depolarization. In particular, $\mathrm{Ca}^{2+}$ currents mediated through $\mathrm{Ca}_{\mathrm{v}} 2 . \mathrm{I}$ channels are facilitated and then inactivated during high-frequency action potentials. This is thought to participate in the observed facilitation and depression of excitatory postsynaptic responses in the calyx of Held (Borst and Sakmann, I998; Forsythe et al., I998).

The experimentally observed $\mathrm{Ca}^{2+}$-dependent regulation of $\mathrm{Ca}_{v} 2$.I channels can be explained through the interaction of calmodulin with these channels (Lee et al., I999). Calmodulin is a $\mathrm{Ca}^{2+}$ sensor protein with two $\mathrm{Ca}^{2+}$-binding sites; a high-affinity site at the amino-terminal lobe and a low-affinity at the carboxyl-terminal lobe of the protein molecule. Mutation of the EF hands at the high-affinity C-terminal predominantly prevents $\mathrm{Ca}^{2+}$-dependent facilitation of $\mathrm{Ca}_{v}$ 2.I channels. In contrast, mutation of the EF hands at the low-affinity $\mathrm{N}$-terminal primarily prevents $\mathrm{Ca}^{2+}$-dependent inhibition of $\mathrm{Ca}_{v} 2$.I channels (Lee et al., I999; Lee et al., 2003). Similarly, the $\mathrm{Ca}_{v} 2 . \mathrm{I}$ channels have two binding sites for calmodulin near the c-terminus of the channel: an IQ-like motif which is essential for $\mathrm{Ca}^{2+}$-dependent facilitation and a calmodulin-binding domain which is required for $\mathrm{Ca}^{2+}$-dependent inhibition (DeMaria et al., 200I).

Based on these observations, a proposed molecular model postulates that when $\mathrm{Ca}^{2+}$ channels open, the initial $\mathrm{Ca}^{2+}$ influx is activating the high-affinity $\mathrm{Ca}^{2+}$-binding site of calmodulin. Calmodulin in turn binds to the IQ-like motif of $\mathrm{Ca}_{\mathrm{v}}$ 2.I channels, causing them 
to facilitate. After a prolonged $\mathrm{Ca}^{2+}$ entry, the $\mathrm{Ca}^{2+}$ concentration is starting to increase which causes the low $\mathrm{Ca}^{2+}$-affinity binding site of calmodulin to be activated, which in turn then interacts with the calmodulin-binding domain of $\mathrm{Ca}_{\mathrm{v}} 2$.I channels, which become inactivated. In the calyx of Held, both $\mathrm{Ca}^{2+}$-dependent facilitation and then $\mathrm{Ca}^{2+}$-dependent inhibition become manifested during a high-frequency stimulation (Forsythe et al., I998).

\section{I.2 THE FUNCTION OF THE SV RELEASE MACHINERY IN DETAIL}

\section{I.2.I Release Probability}

When an action potential arrives at a presynaptic terminal not all synaptic vesicles that are ready to release their contents do it. Indeed, since the early work pioneered by Katz and colleagues in the 50s, it has been established that only a fraction of "units available" would be released (Del Castillo and Katz, I954a, b). By units they were referring to the quanta of neurotransmitter released by individual synaptic vesicles. More specifically, the strength of synaptic output is dependent on four factors: the number of release sites available for accepting a synaptic vesicle $(N)$, the probability that such a site will be occupied by a vesicle $\left(p_{\text {occ }}\right)$, the probability that a vesicle will be successfully released $\left(p_{\text {succ }}\right)$ and finally the quantal size $q$ of a synaptic vesicle. This last measure corresponds to the level of postsynaptic response to the release of neurotransmitter from a single synaptic vesicle. The product of these four factors is the synaptic output $y$ (Neher, 20I7):

$$
y=N \times p_{\text {occ }} \times p_{\text {succ }} \times q .
$$

\section{I.2.2 Synaptic Vesicle Functional Pools}

Synaptic plasticity is largely influenced by calcium. Nanodomains with an elevated calcium concentration around presynaptic voltage-dependent calcium channels trigger vesicle fusion (Schneggenburger and Neher, 2005). As it was mentioned earlier, vesicle fusion is triggered through the activation of synaptotagmin by $\mathrm{Ca}^{2+}$. Additionally, $\mathrm{Ca}^{2+}$ buffers are proteins with rapid $\mathrm{Ca}^{2+}$-binding kinetics, like parvalbumin in the calyx of Held, which intercept the ions be- 
fore they reach the sensors and they can be both mobile and fixed (Muller et al., 2007). When calcium channels close, the calcium nanodomains collapse due to $\mathrm{Ca}^{2+}$ buffers and the diffusion of $\mathrm{Ca}^{2+}$ ions. The remaining calcium is then gradually removed from the presynaptic bouton, but meanwhile it can play an important role in shortterm plasticity (Regehr, 20I2).

Another important aspect in considering presynaptic short-term plasticity is the categorization of synaptic vesicles into functional pools. Almost every synapse has a group of synaptic vesicles that are release-ready. This class is termed the readily-releasable pool (RRP). RRP vesicles are the first to be released during sustained synaptic activity. Consequently, they are also the first to be depleted (Alabi and Tsien, 20I2). In terms of the definition put forward by Neher (20I7), as described earlier, the RRP size is the product $N \times p_{\text {occ. }}$.

In order to quantify the size of the RRP, it is required to have a method of depleting it, an assay to measure the presynaptic output and a method of estimating the replenishment rate. One of the most commonly-used methods of RRP depletion is with a high-frequency stimulation of the afferent fibers. This method has the benefit of resembling physiological conditions, since the electrical stimulation of the axons produces action potentials which are propagated along its length. However, it is spread over time; a typical $50-$ pulse $100-\mathrm{Hz}$ stimulation burst lasts $500 \mathrm{~ms}$. This means that the ongoing recruitment of new vesicles may make it impossible to fully deplete the RRP. In consequence, a considerable amount of the measured response could be from newly-recruited synaptic vesicles (Neher, 2015).

If a train is plotted in a cumulative fashion versus the stimulus number, then the final data points would form a straight line as the rate of depletion would be equal to the rate of replenishment. In consequence, the procedure to estimate the RRP size from such graphs is relatively straightforward, according to Schneggenburger et al. (I999); a regression line is fitted to the straight portion of the graph and then back-extrapolated so that it intercepts the $y$-axis. The point of intersection reports the RRP size, whereas the slope of the regression line represents the replenishment rate. Although, this method is not reporting the actual size of the RRP but only its decrement during a train, when the stimulation is strong enough so that the EPSCS during a train depress significantly more than $50 \%$ the error can be 
quite small. For a formal treatment of the limits of this method and the corrections that can be applied, the reader is encouraged to read E. Neher's review (2015).

Another way of depleting the RRP is through the brief application of a hypertonic solution to the neuronal preparation. Usually this is done through the addition of $\sim 500 \mathrm{mOsm} / \mathrm{L}$ of sucrose to the extracellular medium (Rosenmund and Stevens, 1996). This method has been widely used in hippocampal cultures where the extracellular medium can reach the synapses relatively fast.

When the RRP is depleted, release continues from the recycling pool which is comprised of the recycled synaptic vesicles during an intense or prolonged physiological stimulation (Rizzoli and Betz, 2005). Typically, the recycling pool size is three times larger than the readily-releasable pool. This fraction remains stable independently of the recycling pool size, as measured in hippocampal cultures (Murthy and Stevens, 1999).

Finally, the rest of the synaptic vesicles which also constitute the majority in a presynaptic terminal (50-85\%) belong to the reserve pool (Alabi and Tsien, 20I2). These vesicles are not released even after an intense or prolonged stimulation and can be detected through their inability to release genetically encoded synaptopHluorin probes (Li et al., 2005). A pool that approximates the above definition of a reserve pool has been described also for the calyx of Held (de Lange et al., 2003).

\section{I.2.3 Short-Term Plasticity}

Most synapses when they undergo a period of prolonged activity they exhibit a change in presynaptic strength which can last from tens of milliseconds to minutes. This change in synaptic weight was observed for the first time more than 70 years ago (Eccles et al., I94I). According to the direction of the presynaptic strength this plasticity can be a depression, a facilitation or a post-tetanic potentiation (Zucker and Regehr, 2002). Usually, a presynaptic depression or facilitation is apparent even from the second of two closely spaced stimuli and depending on the strength of the stimulation, it can persist for minutes. However most of the times, multiple forms of plasticity are occurring at the same time in the presynaptic terminal and the 
interaction of these forms is reflected in the net synaptic strength (Dittman et al., 2000).

Many synapses display a decrease in synaptic strength when they are intensely activated. The most prominent mechanism of synaptic depression lies in a decrease of neurotransmitter release from the presynaptic terminal which appears to be due to a depletion of synaptic vesicles from the RRP. Additionally, feed-back inhibition through modulatory substances from the presynaptic and postsynaptic terminals, can also result in synaptic depression. Finally, desensitization and saturation of the postsynaptic neurotransmitter receptors can reduce the effect of the exocytosed neurotransmitter to the postsynaptic cell.

The simplest model to explain paired-pulse depression is the depletion model of depression (Zucker and Regehr, 2002). It postulates that during the first stimulus an $F$ fraction of the $S$ total vesicles in the RRP is released, producing a current $i$ in the postsynaptic cell. Then, this current has an EPSC amplitude of $A_{I}=F S i$. When the second stimulus arrives the RRP size is reduced and only $S-F S$ vesicles are available. Thus, the second EPSC amplitude will be $A_{2}=S(I-F) F i$ and the paired pulse ratio will be $A_{2} / A_{I}=I-F$.

It is important to note that this model makes the following three assumptions; the RRP fraction that is released with the first stimulus is large, there is no replenishment of synaptic vesicles between the first and the second pulse and the release probability is the same at both stimulations.

The depletion model predicts that the paired-pulse depression is proportionate to the initial probability of release; the larger the probability of release, the more synaptic vesicles are being released with the first stimulus and less vesicles are available for the second one, thus the stronger the paired-pulse depression. This negative correlation between the amplitudes of the first and the second EPSC has been shown for a plethora of synapses, including the calyx of Held (Scheuss et al., 2002). In some synapses however, like the hippocampal synapses in cultures, the depletion model cannot fully explain the observed paired-pulse depression (Chen et al., 2004).

When a synapse undergoes a prolonged high-frequency activity then the subsequent depression can last much longer, up to tens of seconds. This is thought to be occurring due to depletion of the re- 
cycling pool. In hippocampal autapses, which are cultured neurons that grow in isolated "islands" so that they can make synaptic contacts only with themselves, a 9-Hz Iooo-stimuli train was required to observe a depletion of the recycling pool (Stevens and Wesseling, I999).

Lastly, the role of reduced $\mathrm{Ca}^{2+}$ entry in synaptic depression should not be understated. In presynaptic recordings from the calyx of Held it was found that a reduction of $\mathrm{P}$-type $\mathrm{Ca}^{2+}$ current contributes to posttetanic (Ioo $\mathrm{Hz}$ for Io s) depression of excitatory transmission (Forsythe et al., I998). Additionally, a $\mathrm{Ca}^{2+}$-current reduction can account for the paired-pulse depression seen at inter-stimulus intervals longer than Ioo $\mathrm{ms}$ and for the short-term depression after a low frequency $(\leq 30 \mathrm{~Hz})$ stimulation $(\mathrm{Xu}$ and $\mathrm{Wu}, 2005)$.

One of the first models trying to explain presynaptic facilitation was the residual calcium hypothesis (Katz and Miledi, I968). It predicts that the $\mathrm{Ca}^{2+}$ that is entering in a cell during a presynaptic depolarization is not fully removed before a second closely-spaced stimulus arrives; thus, the $\mathrm{Ca}^{2+}$ that enters the presynaptic terminal during the second stimulus is added up to the residual $\mathrm{Ca}^{2+}$ from the first stimulus. This results in facilitation, provided that the residual $\mathrm{Ca}^{2+}$ is significantly high. However, a formal treatment of this hypothesis along with experimental data for the local $\mathrm{Ca}^{2+}$ concentration at the calyx of Held, suggest that this model cannot adequately explain paired-pulse facilitation, since residual $\mathrm{Ca}^{2+}$ would provoke only a $4 \%$ enhancement (Regehr, 20I2).

Nevertheless, the residual $\mathrm{Ca}^{2+}$ could indeed promote facilitation by acting on a $\mathrm{Ca}^{2+}$ sensor, different than synaptotagmin-I (Atluri and Regehr, 1996). In particular, this sensor would need to be high-affinity and slow-acting so that it would be able to sense the low concentrations of residual $\mathrm{Ca}^{2+}$ and promote facilitation in the long run. In contrast, synaptotagmin-I is low-affinity and fast-acting.

In addition, a high-affinity $\mathrm{Ca}^{2+}$ buffer in the presynaptic terminal, not unlike BAPTA, can help facilitation. This kind of buffer would associate with calcium entering the terminal during the first stimulus and the free buffer's concentration would be smaller during the second stimulus. Thus, there would be a higher local calcium elevation during the second pulse and a paired-pulse facilitation would appear (Neher, I998). 
Finally, a use-dependent modulation of voltage-gated $\mathrm{Ca}^{2+}$ channels through $\mathrm{Ca}^{2+}$ sensor proteins and slow transmitter systems would also enhance facilitation (Takahashi, 2005). At synapses between cultured superior cervical ganglion neurons blocking the facilitation of $\mathrm{P} / \mathrm{Q}$ type $\mathrm{Ca}^{2+}$ currents that is dependent on $\mathrm{Ca}^{2+} / \mathrm{Ca}^{2+}$ sensor proteins, also markedly reduced the facilitation of synaptic transmission (Mochida et al., 2008).

\section{I.2.4 Multiple SV Priming States}

So far, the synaptic vesicle release processes have been described as stable and static states; a synaptic vesicle is docked and primed at one of the active zone release sites and then, when an action potential (AP) arrives, the intracellular calcium concentration is increased and the synaptic vesicle fuses with the active zone membrane with a certain release probability. However, different subsets of synaptic vesicles (svs) that mediate transmission have been reported for a plethora of synapses, including the calyx of Held, cerebellar mossy fibers to granule cells, hippocampal neurons and parallel fibers to molecular-layer interneuron synapses.

In particular, electrical stimulation of the calyx of Held revealed two components of the fast-release pool with different release probabilities and replenishment rates. The fast pool was described as having a high release probability and a slow replenishment rate whereas the slow pool seemed to be replenished quickly but having a much lower release probability (Taschenberger et al., 20I6). In another study, the refilling of the fast pool occurred at the expense of the slower one (Lee et al., 20I2), pointing to a linear "maturation" of the svs where the fast svs have to go through the slow stage first.

In the cerebellar mossy fiber to granule cell synapses, a comparable two-step priming process has been described, where the slow component was recovering with a time constant of a few seconds (Hallermann et al., 20I0). In hippocampal neurons a subgroup of synaptic vesicles that are more likely to be released in the first few APs of a stimulus train was described with the term "superprimed". The superprimed svs had a higher release probability and slower replenishment rate than their normally-primed counterparts (Schluter et al., 2006). 
At individual synapses between presynaptic parallel fibers and postsynaptic molecular-layer interneurons an SV renewal process that was dependent on previous release events was described; release events during the first AP of a stimulus train were mediated through a well synchronized, fast component. However, during subsequent APS at $200 \mathrm{~Hz}$ a slow, desynchronized component was gradually developing. In addition, the speed of transition from the slow to the fast pool of sVs was increased during the AP train, possibly due to enhanced $\mathrm{Ca}^{2+}$ entry and an augmented intracellular calcium concentration transient (Miki et al., 20I8). This transition was hindered by the actin polymerization inhibitor latrunculin B (Miki et al., 20I6; Miki et al., 20I8). In contrast to the previous models, here a sequential model was sufficient to explain the observed data; svs were recruited from a recycling pool, and went through a "replacement site" before maturing to a "docking site" where they could be released. Thus, only one release probability was assigned to svs.

All of the aforementioned studies had to postulate at least two states of primed svs in order to explain their experimental observations. Most were suggesting that Sv release can occur from both states; both the slowly-primed and the fast svs were release capable, while the number of the sv docking sites was unchanged (Hallermann et al., 20Io; Schluter et al., 2006; Taschenberger et al., 2016).

Newer studies, however, are suggesting a dynamic equilibrium in docked and primed synaptic vesicles. As mentioned before, Syt-I mutants with no ability to bind to anionic lipids or the SNARE complex failed to bring svs in close proximity to the active zone. After a single AP, though, svs were rapidly recruited to the membrane, a step which was $\mathrm{Ca}^{2+}$-dependent and almost completely reversed after Ioo ms (Chang et al., 20I8). Similarly, non-functional Munci8-I caused a significantly reduced basal transmission but high-frequency stimulation greatly facilitated the EPSCs. Again, this facilitation was rapidly falling back to the rested-state levels shortly after the end of the stimulus train, also in Munci3-I deficient synapses (He et al., 20I7).

This separation of vesicles into two populations that may also be in a dynamic balance, gave rise to the question of what is the mechanism that distinguishes these populations. The positional priming theory posits that the state of a SV depends on its proximity to the $\mathrm{Ca}^{2+}$ source. Vesicles that are positioned close by a $\mathrm{Ca}^{2+}$ channel have 
a high release probability. On the other hand, svs that are further away, although they are also release competent, cannot "sense" the increased calcium concentration because the increase is restricted to the nanodomains around the channels during a short influx episode. These vesicles undergo a positional change which brings them closer to the calcium sources, and thus they are rendered release-capable (Meinrenken et al., 2002). This step is strongly dependent on $\mathrm{Ca}^{2+}$ (Neher and Sakaba, 2008).

If the sole defining difference between a slow and a fast vesicle was its distance from the $\mathrm{Ca}^{2+}$ source and not its intrinsic calcium sensitivity, then if the intracellular $\left[\mathrm{Ca}^{2+}\right]$ was uniformly elevated throughout the terminal at once only a single component would be observed. However this was not the case when presynaptic $\mathrm{Ca}^{2+}$-uncaging measurements were made at the calyx of Held. Deconvolution analysis of the resulting time course of transmitter release revealed a fast and a slow component over a wide range of $\mathrm{Ca}^{2+}$ concentrations; this indicated that the difference should be due to a property of the fusion machinery itself (Wolfel et al., 2007).

Subsequent research reconciled these two theories, through a mechanism where synaptic vesicles first move to the active zone and become part of the fast releasing pool and then they attain a high $\mathrm{Ca}^{2+}$ sensitivity for fusion. The first part seemed to be regulated by actin- and PLC-dependent mechanisms, while the second was regulated by PLC-mediated mechanisms (Lee et al., 2013). Thus, the svs with a high $\mathrm{Ca}^{2+}$ sensitivity have a high probability of release and they are termed "superprimed", whereas the rest of the svs have a lower release probability (Schluter et al., 2006; Taschenberger et al., 2016).

A more elegant explanation comes by Neher and Brose (2018) with the help of the multifaceted SNARE complex and its associated proteins. Their model manages to incorporate the recent data on stimulation-dependent priming and subsequent "de-priming" that were previously described, while successfully predicting the behavior of an entire range of synapses: from those with high initial release probability, like the calyx of Held, to the ones with low initial probability of release like the hippocampal mossy fibers.

The authors propose that the fast reloading of the primed sv pools during high-frequency synaptic activity is due to $\mathrm{Ca}^{2+}$-dependent rapid transitioning of the SNARE complex from a loose state (LS) to a 
tight docking state (TS) which enables the vesicles to be released. This tight state requires SNARES along with Munci3-I and Munci8-I and additionally Syt-I. When any of these proteins are absent then the svs fall back into a precursor "loose" state, where the SNARE complex is partially unzippered. Additionally, a high intracellular $\mathrm{Ca}^{2+}$ concentration stabilizes the svs into the tight state.

In summary, the model postulates that as the synapse is at rest there are three populations of svs; the Ts vesicles, the LS vesicles and the synaptic vesicles that are in the replacement pool. Naturally, the TS and LS svs are docked into a fixed number of release sites. It is important to note that not all release sites are occupied with Ts vesicles. The probability that a release site is occupied by a TS vesicle is $<$ I for typical depressing synapses like the calyx of Held and could be as low as $<0.2$ for a facilitating synapse like the cerebellar granule cell to Purkinje cell synapse. Therefore, svs exist in a dynamic balance between a loose and a tight state.

When the first action potential of a high-frequency stimulation train arrives, the first svs to be released with a certain release probability $\left(p_{T S}\right)$ are the ones who are in the TS state. As the second AP arrives, there are now two Sv populations in the TS state that will get released: the TS Svs that did not get released during the first AP and newly-formed TS vesicles that transitioned from the LS state due to the concomitant increase in intracellular $\mathrm{Ca}^{2+}$. Additionally, an increase in $p_{T S}$ cannot be ruled out, due to intracellular $\mathrm{Ca}^{2+}$ buildup (Neher, 20I7).

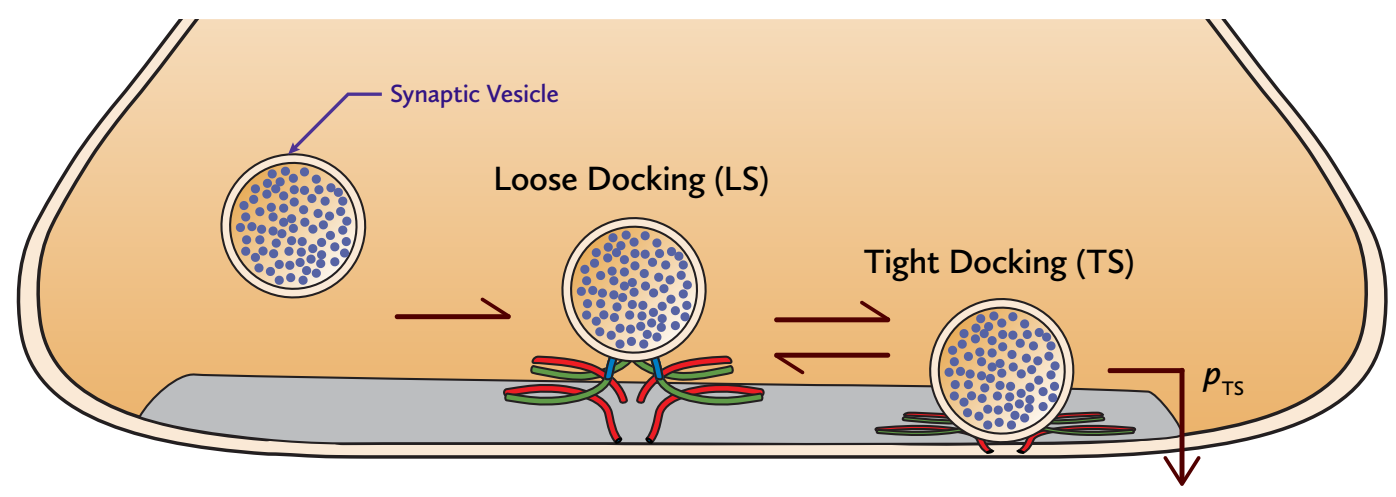

FIGURE 1: The three synaptic vesicle states predicted by the loose-tight state model. An SV is recruited to the active zone, then transitions from a loose to a tight state and is fused with the presynaptic membrane with a certain release probability. 
During the next few APs of the high-frequency train the following events occur; the Ts vesicles (that is, the svs that were in the tight state before the beginning of the train) will get gradually depleted as they fuse with each AP; the fusing vesicles that were in the loose state in the beginning of the train will see their numbers rise as the Lsto-TS transition rate will be enhanced due to increased $\mathrm{Ca}^{2+}$, but then this population will decline as the LS pool will get depleted. Gradually, a third component will take over; it will be comprised of svs that are newly recruited (RS) during the stimulation train and they will go through the loose and then the tight state before they fuse.

\section{I.3 THE CALYX OF HELD}

The calyx of Held is a giant axosomatic synapse in the auditory pathway; the postsynaptic neuron provides inhibition in most nuclei of the auditory brainstem. Due to the synapse's size, which permits direct presynaptic patch-clamp recordings, it has become a popular model among neuroscientists for the study of mechanisms of transmitter release.

Its name is owed to the German anatomist Hans Held from the university of Leipzig, who used the Golgi technique to stain and study the central auditory pathway in cats (Held, I893). A few years later Ramón y Cajal, the Spanish histologist, further studied these synapses, producing the first detailed images of the calyx (Ramón y Cajal, I909). Immunohistochemical studies showed terminal-like elements immunoreactive to a glutamate antibody, covering the perikarya of the postsynaptic neurons: the medial nucleus of the trapezoid body principal neurons (Grandes and Streit, I989).

Electrophysiological recordings confirmed the glutamatergic nature of the calyx; they showed a complete block of calyceal EPSPS after using the AMPA/kainate receptor antagonist CNQX (Banks and Smith, 1992). It was also found that the EPSCs can be split into two components: a fast time-course AMPA-mediated component and a slower NMDA-receptor mediated component (Forsythe and Barnes-Davies, 1993). Since then, the accessibility of the calyx of Held in both in vivo and in vitro brain slice preparations has been used to study glutamate release, presynaptic ion channels, $\mathrm{Ca}^{2+}$ influx and short-term plasticity. 
Axons originating mainly from the globular bushy cells of the anteroventral cochlear nucleus project to the medial nucleus of the trapezoid body (МNTB), which is part of the superior olivary complex, forming calyces. Each axon forms a single giant axosomatic calyx on a principal MNTB cell (Morest, I968), although in 20\% of the cases they can form two calyces on separate MNTB neurons (Rodriguez-Contreras et al., 2006). Interestingly, the MNTB principal neurons receive somatic inhibitory synapses the origin and function of which remain unknown (Awatramani et al., 2004). The number of MNTB cells ranges from 3,000 to 7,000 in most mammals (Kulesza et al., 2002) and they project to other superior olivary complex nuclei, including the lateral superior olive, forming inhibitory glycinergic synapses (Tollin, 2003).

The morphology of the active zones of the calyx of Held is similar to that found in an ordinary synapse; they have a surface area which is similar to hippocampal excitatory synapses and they contain two to three docked synaptic vesicles (Satzler et al., 2002; Taschenberger et al., 2002). Where the calyx differs however, is in the number of active zones per synapse; typical terminals in the brain usually have one active zone per synapse, whereas the calyx of Held has about 300-700 active zones until postnatal day I4 (Taschenberger et al., 2002). In this way, the arrangement of the active zones of the calyx of Held can be considered having a "parallel" arrangement, which can be driven by a single presynaptic action potential (Schneggenburger and Forsythe, 2006).

The calyx of Held undergoes several developmental changes until it reaches a fully functional state, at the time of the hearing onset in the animal. Most studies in this synapse have been done in rodents, especially rats, before hearing onset as it becomes progressively harder to access the calyx for presynaptic recordings after that age, due to increased myelination.

$\mathrm{Ca}^{2+}$ entry in the calyx terminal is mediated by voltage-dependent $\mathrm{N}-, \mathrm{R}-$ and $\mathrm{P} / \mathrm{Q}-$ type $\mathrm{Ca}^{2+}$ channels; however, $\mathrm{P} / \mathrm{Q}$-type channels are more efficient in coupling $\mathrm{Ca}^{2+}$, suggesting their placement in the active zones. Moreover, paired-pulse facilitation is greatly diminished when $\mathrm{P} / \mathrm{Q}$-type channels are knocked out (Inchauspe et al., 2004). When intraterminal $\mathrm{Ca}^{2+}$ is increased, neuronal $\mathrm{Ca}^{2+}$ sensor I (Tsujimoto et al., 2002) and calmodulin (Nakamura et al., 2008) can me- 
diate an activation of $\mathrm{P} / \mathrm{Q}$-type $\mathrm{Ca}^{2+}$ channels (Inchauspe et al., 2004); this activation can, in turn, facilitate the $\mathrm{Ca}^{2+}$ current entering the terminal during a subsequent action potential (Cuttle et al., I998).

Transmitter release is controlled by $\mathrm{Ca}^{2+}$ ions. The relation, however, between intracellular calcium concentration and transmitter release is supralinear; from 0.3 to I $\mathrm{mM}$ of extracellular $\mathrm{Ca}^{2+}$ concentration, transmitter release scales with the second power of the calcium concentration (Barnes-Davies and Forsythe, 1995). At intracellular $\mathrm{Ca}^{2+}$ concentrations of $2-5 \mu \mathrm{M}$, achieved with flash photolysis, the relation is scaled to the fourth or fifth power (Schneggenburger and Neher, 2000). This highly non-linear relationship between calcium concentration and transmitter release can also be seen in the fact that whereas at $2 \mathrm{mM}$ of extracellular $\mathrm{Ca}^{2+}$ about $2 \mathrm{IO}$ quanta are released, at one eighth of that concentration there is not a single quantum detected during most action potentials (Borst and Sakmann, 1996).

As detailed earlier, the term quantal size refers to the amplitude of the postsynaptic response to the neurotransmitter released by the presynaptic fusion of one vesicle. At the calyx of Held, it has been measured from spontaneous EPSCs at around 30-35 pA, at room temperature and a holding potential of $-70 \mathrm{mV}$ (Chuhma and Ohmori, I998; Sahara and Takahashi, 200I).

Upon the arrival of an action potential at the presynaptic terminal, the ensuing EPSC recorded at the postsynaptic neuron is in the range of 4-8 $\mathrm{nA}$, sometimes reaching up to $15 \mathrm{nA}$ (Schneggenburger and Forsythe, 2006). Many attempts have been made to estimate the quantal content of a single EPSC; in some cases it has been estimated by dividing the EPSC amplitude by the quantal size (Borst and Sakmann, I996; Schneggenburger et al., 1999). In other cases, variance-mean analysis was employed in order to bypass the potential pitfall of glutamate spilling over to neighboring synapses and thus adding up quanta non-linearly (Meyer et al., 200I; Taschenberger et al., 2005). Both approaches yielded the same estimate of $150-200$ quanta released during a single action potential of 4-8 nA.

The immature calyx under repetitive stimulation undergoes a strong synaptic depression of its EPSCS, when being under standard brain slice recording conditions. These conditions, typically include $2 \mathrm{mM}$ of calcium in the extracellular solution. The observed depres- 
sion is mainly due to depletion of synaptic vesicles from the RRP (Wu and Borst, 1999). Using the back-extrapolation method developed by Schneggenburger, Meyer and Neher, detailed in chapter 1.2.2, synaptic vesicles belonging in the RRP were estimated at 60o-80o (Bollmann et al., 2000; Schneggenburger et al., 1999). However, when directly stimulating the presynaptic terminal using a prolonged depolarization, deconvolution revealed an RRP estimate of $\sim 2,400$ svs (Sakaba and Neher, 200I). Similarly, presynaptic capacitance recordings which measure the increase in the presynaptic capacitance induced by the fusion of svs, produced an RRP estimate of $\sim 4,000$ Svs (Sun and Wu, 200I; Wolfel and Schneggenburger, 2003). Finally, $\mathrm{Ca}^{2+}$-uncaging-evoked release assigned about 3,600 svs in the calyx's RRP (Wolfel et al., 2007). Thus, it can be a safe assumption that the calyx of Held has a readily-releasable pool size of $\sim 3,000$ synaptic vesicles.

Synaptic depression is not the only expression of short-term plasticity in the calyx of Held; facilitation of the second EPSC during a high-frequency stimulation train is also common in a subset of synapses (Taschenberger et al., 20I6). This facilitation, however, is not dependent on an altered intracellular $\mathrm{Ca}^{2+}$ sensitivity of transmitter release (Felmy et al., 2003). In addition, following a prolonged train of presynaptic action potentials transmitter release can be enhanced for several minutes afterwards. This post-tetanic potentiation is dependent on residual $\mathrm{Ca}^{2+}$ (Habets and Borst, 2005) that builds up gradually during the stimulation train and originates from mitochondria (Lee et al., 2008). No longer-lasting forms of plasticity have been described at the calyx of Held.

\section{I.4 MOVER}

Among the evolutionarily conserved proteins of the presynaptic machinery, lie a handful which were introduced quite late in the neurotransmitter release apparatus and thus they are vertebrate specific. One of them, which will also be the focus of this study, is Mover.

Mover is a 266 amino acid presynaptic protein, homologues of which exist only in vertebrates. It interacts with the also vertebrate-specific protein Bassoon, in the yeast-2-hybrid assay (Kremer et al., 2007). Additionally, in the endbulbs of Held, the first central syn- 
apses of the auditory system, Mover is downregulated in excitatory presynaptic terminals when functional Bassoon is absent from the synapse (Mendoza Schulz et al., 20I4).

Furthermore it is associated with synaptic vesicles as a peripheral membrane protein, since it lacks a transmembrane domain. This SV association requires that Mover is phosphorylated, as globally dephosphorylating synaptic proteins dissociates Mover from svs (Ahmed et al., 2013). An additional requirement for Mover's presynaptic sv targeting is the homomeric interaction it undergoes (Ahmed et al., 2013) possibly through its hSac2 domain (Hsu et al., 2015).

A surprising characteristic of Mover is its heterogeneous distribution throughout the brain, considering the relatively unchanged components of the presynapse across different synapses. In particular, Mover seems to be upregulated in the septal nuclei, ventral pallidum and amygdala, while it is in quite low concentrations in the granular layer of the cerebellum, primary somatosensory cortex and medial habenula (Wallrafen and Dresbach, 2018). In the hippocampus, Mover has an increased presence in the stratum radiatum and stratum oriens of the CAI region and also in the stratum radiatum and stratum lucidum of CA3; both regions are rich in synaptic contacts. More specifically, it seems to be present only in excitatory synapses in the CA3 region, while absent from the inhibitory terminals. Additionally, Mover is downregulated at the pyramidal layers of the hippocampus, where the cell bodies of pyramidal neurons lie (Kremer et al., 2007; Wallrafen and Dresbach, 2018).

In the auditory pathway Mover is found in both excitatory and inhibitory terminals at the endbulbs of Held (Mendoza Schulz et al., 20I4). Accordingly, in the calyx of Held Mover is present in the presynaptic terminal and absent from the soma of the postsynaptic cell (Korber et al., 2015; Kremer et al., 2007).

In terms of function, little is known about Mover's effect on synaptic transmission. In a knock-down model of Mover in the rat calyx of Held, EPSC amplitudes were increased, paired-pulse ratio decreased and short-term depression was faster and more complete. In addition, $\mathrm{Ca}^{2+}$-uncaging experiments showed slightly higher evoked EPSC amplitudes in one of the low-calcium conditions. Based on these findings, the authors proposed an increased release probability upon Mover's disruption in this synapse (Korber et al., 2015). 


\section{I.5 Aim OF THIS STUDY}

Recent results on the function of Mover in a knock-down rat model, indicated an increase in $\mathrm{Ca}^{2+}$ sensitivity of release (Korber et al., 20I5). In this light, I seek out to elucidate the function of Mover in neurotransmission using a Mover ko mouse line and a central glutamatergic synapse as a paradigm, the calyx of Held. This synapse has been established as a model for studying presynaptic mechanisms of neurotransmitter release. To perform this study, I used electrophysiology in auditory brainstem slices; in particular I will be electrically stimulating the afferent axons reaching the medial nucleus of the trapezoid body while simultaneously patch-clamping a principal neuron of this nucleus. Thus, this study will examine Mover's influence on basic synaptic transmission and short-term plasticity trying taking into consideration the recent advances in our understanding of synaptic vesicle priming. In a more general view, it will be further clarified whether Mover acts as an essential part or as a modulator, if at all, in the evolutionarily highly conserved presynaptic machinery. 


\section{I. ANIMALS}

For this study, juvenile (PI2 - PI3) mice (Mus musculus) of either sex were used and they were wild-type (WT) and Mover knockout (Kо) littermates. The animals were bred and maintained at the main animal facility of the University Medical Center of Goettingen. The generation of the mouse line is described in the thesis of Dr. Friederike Wetzel (Wetzel et al.), and it was maintained through heterozygous breeding. Mice were genotyped before an experiment and regenotyped afterwards, as detailed in the thesis of Dr. Julio Viotti (Viotti, 20I8). Genotyping was performed by Irmgard Weiss.

All experiments complied with the guidelines of the University of Göttingen and the state of Lower Saxony (Landesamt für Verbraucherschutz, Braunschweig, Germany).

\subsection{SLICE PREPARATION}

Slices were prepared in artificial cerebrospinal fluid (ACSF) containing (in mM) I25 NaCl, $2.5 \mathrm{KCl}, 25 \mathrm{NaHCO}_{3}, \mathrm{I} .25 \mathrm{NaH}_{2} \mathrm{PO}_{4}$, Io glucose, 0.4 ascorbic acid, 3 myo-inositol, 2 Na-pyruvate, $3 \mathrm{MgCl} 2$ and o.I $\mathrm{CaCl}_{2}$. The ACSF solution was freshly prepared before the experiment using the following stock solutions: a solution which contained $\mathrm{NaCl}, \mathrm{KCl}$ and $\mathrm{NaH}_{2} \mathrm{PO}_{4}\left(\mathrm{H}_{2} \mathrm{O}\right)$ in a tenfold concentration and stored at $4^{\circ} \mathrm{C}$ and a separate stock solution which contained ascorbic acid, myo-inositol and Na-pyruvate in a hundredfold concentration and stored at $-20^{\circ} \mathrm{C}$. Glucose and $\mathrm{NaHCO}_{3}$ were added separately. Afterwards, the solution was gassed with carbogen (95\% $\left.\mathrm{O}_{2}, 5 \% \mathrm{CO} 2\right)$ for I5 minutes and $\mathrm{MgCl}_{2}$ and $\mathrm{CaCl}_{2}$ were added from separate stock solutions of I $\mathrm{M}$ of $\mathrm{MgCl}_{2}\left(6 \mathrm{H}_{2} \mathrm{O}\right)$ and $\mathrm{CaCl}_{2}\left(2 \mathrm{H}_{2} \mathrm{O}\right)$ respectively, stored at $4{ }^{\circ} \mathrm{C}$.

The animal was decapitated rostral to the first cervical vertebra. Using a standard scissors a cut through the scalp and the cutane- 
ous muscle was made, starting from the occipital bone and running rostrally to the nasal bone. Attention was given to fully expose the cranial sutures on the dorsal surface of the skull.

Afterwards, the skull was cut through using small scissors. The cutting edge of the lower blade of the scissors was placed inside the superior region of the foramen magnum. Next, keeping the lower blade firmly against the inner surface of the occipital plate a cut was made through the midline suture of the parietal bone and then through the frontal bone. Two more cuts were made at the sutures between the parietal and the frontal bone, starting from the midline and proceeding laterally at both sides.

The occipital and parietal bones were then separated from the scull using forceps. This exposed the dorsal surface of the brain. Using microscissors (cat. no.: 15003-08; Fine Science Tools, Canada), the brain was extracted. Specifically, the microscissors were inserted between the anteroventral surface of the brain and the skull plates and pushed the brain away from the skull in order to expose the intact cranial nerves. Then, these nerves were cut using the microscissors, starting from the anterior ones and proceeding posteriorly until the brain freely fell inside a petri dish with $\mathrm{Ca}^{2+}$-free ASCF at $5^{\circ} \mathrm{C}$ which was continuously gassed with carbogen. Care was taken in order not to pull the brain away from the skull at any point, as this would damage the brainstem.

The whole process, from decapitation until the immersion of the brain in ASCF lasted less than 60 seconds. Longer lasting extractions can inadvertently impact in a negative way slice health (Bischofberger et al., 2006).

The meninges surrounding the brain stem were subsequently removed. The petri dish with the whole brain was transferred under a low-magnification stereomicroscope (Stemi DV4; Zeiss, Germany). Two fine forceps (cat. no.: II25I-35; Fine Science Tools, Canada) were employed to remove the meninges while the ventral surface of the brain was facing upwards; one forceps was used to pin down the brain at the cerebrum and keep it steady; with the other forceps the edge of the meninges were taken hold of at the anterodorsal brain stem and pulled towards the ventral part of the structure. This step was essential to ensure proper slicing afterwards. 
Then, the cerebrum was cut in half to provide a flat surface to glue the brain on the microtome chuck. The brain was turned again so that the dorsal surface was facing upwards and a lateral cut was made in the middle of the medial line using a scalpel with a No. 22 blade. The anterior part of the brain was facing leftwards and the angle of the cut was at $45^{\circ}$, when tilting the upper part of the blade to the right. It was crucial to cut at this angle because that ensured that the afferent axon connections to the MNTB principal cells that were close to the surface of the slice - and thus accessible to patching - would not be severed. These axons were the ones that later would be electrically stimulated.

The next step was the gluing of the brain on the microtome chuck and preparing it for slicing. A drop of super glue (Ultra Gel; Pattex, Germany) was placed on the microtome plate and then spread around using a piece of tissue paper. The brain was then placed in the middle of the chuck and a few drops of $\mathrm{Ca}^{2+}$-free ASCF were dropped on top of it. This step was essential so that the rest of the glue that was on the chuck would not creep around the brain when filling the cutting dish with ACSF. Afterwards the chuck was placed inside the buffer tray and it was slowly filled with carbogen-gassed $\mathrm{Ca}^{2+}$-free ASCF until the entire brain was covered by it. The buffer tray along with its cooling element and 5-7 $\mathrm{ml}$ of $\mathrm{Ca}^{2+}$-free ASCF in it were previously frozen at $-20^{\circ} \mathrm{C}$. During slicing, the temperature of the ACSF in the buffer tray was continuously monitored and kept within $4-6^{\circ} \mathrm{C}$, by placing the measuring tip of an electronic thermometer (GTHII7O; Greisinger, Germany) in the ACSF next to the glued brain piece.

For slicing, a vibrating microtome (Microm HM 650v; Thermo Scientific, USA) was used as it minimizes damage to the upper and lower surfaces of the slice where the cells that are going to be patched are situated. Slices of $275 \mu \mathrm{m}$ thickness were prepared, using a vibrating frequency of $100 \mathrm{~Hz}$ and a cutting speed of $0.8 \mathrm{~mm} / \mathrm{s}$. Since the anterior side of the brain was glued on the chuck, the inferior part of the brainstem was facing upwards. Thus, while slicing the appearance of the 7 th (facial) nerve on the newly cut surface was the visual cue to collect this and the next $2-3$ slices. The 7 th nerve appeared as two white diagonal lines starting near the 4 th ventricle and extending laterally until the edge of the brainstem. These slices were the ones 
that contained the trapezoid body where the MNTB principal cells are situated.

The slices were collected using a Pasteur glass pipette and put into a holding chamber where they were bathed in $\mathrm{Ca}^{2+}$-containing ASCF at $35^{\circ} \mathrm{C}$ for $30-45$ minutes and at room temperature $\left(22.5 \pm \mathrm{I}{ }^{\circ} \mathrm{C}\right)$ thereafter. The $\mathrm{Ca}^{2+}$-containing ASCF was different from the $\mathrm{Ca}^{2+}$-free ASCF only in the following (in $\mathrm{mM}$ ): $\mathrm{I} \mathrm{MgCl}_{2}$ and $2 \mathrm{CaCl}$. It was also freshly prepared before each experiment, as previously described. Additionally, the osmolality was adjusted at 308-3I2 mOsm/ $/ \mathrm{kg}$ (3320; Advanced Instruments, USA) by either adding $\mathrm{H}_{2} \mathrm{O}$ to lower the osmolality or by adding glucose to increase it. If the starting osmolality was less than $303 \mathrm{mOsm} / \mathrm{kg}$ or more than $316 \mathrm{mOsm} / \mathrm{kg}$ then the ACSF was discarded and a new one was prepared.

\subsection{ELECTROPHYSIOLOGY}

Slices were placed in a submerged-type chamber where they were continuously perfused at I-I.5 $\mathrm{ml} / \mathrm{min}$ with carbogen-saturated ACSF. Samples were kept at $25^{\circ} \mathrm{C}$ by using an inline ACSF heater and a chamber heater (TC-2O-WI; npi, Germany). The cells were visualized using a BX5IWI upright microscope (Olympus, Japan) with a 40x water immersion objective.

For stimulating the bushy-cell axons innervating the medial nucleus of the trapezoid body principal cells, a parallel bipolar electrode (cat. no.: PBSAO275; FHC, USA) was placed close to midline and was used to deliver electrical pulses through an isolated current stimulator (Model DS3; Digitimer, UK).

Postsynaptic recordings were performed using pipettes made out of thick-walled borosilicate glass (cat. no.: GBI5OF-8P; Science Products, Germany) with an open tip resistance of I.6-2.6 M $\Omega$. They were pulled always on the day of the experiment using a P-Iooo Pipette Puller (Sutter Instruments, USA). The pipette solution contained (in $\mathrm{mM})$ : Io $\mathrm{KCl}$, I30 K-gluconate, Io hepes, Io TEA-Cl, 5 Na2-phosphocreatine, 5 EGTA, 4 Mg-ATP, 0.3 Na2-GTP. The pH was adjusted to 7.2 with the addition of $\mathrm{KOH}$. The osmolality was at $300-303 \mathrm{mOsm} / \mathrm{kg}$.

Cells were clamped at $-70 \mathrm{mV}$ throughout the experiment and series resistance ranged from 3 to $6.5 \mathrm{M} \Omega$ which was compensated for as much as it was possible without the clamp becoming unstable. 
Spontaneous post-synaptic currents were recorded without series compensation. Current leak was below -50 pA. Postsynaptic currents were recorded using an EPC-IO USB amplifier (HEKA, Germany) and digitized using the data acquisition program PatchMaster (HEKA, Germany). Every postsynaptic response for a particular stimulation protocol was sampled 3-4 times and then averaged.

For recordings using cyclothiazide (CTZ) and kynurenic acid (KYN), the slices were perfused at least $3 \mathrm{~min}$. before the recording. When a cell was patched and evoked EPscs could be elicited, the perfusion with the aforementioned drugs would start. Then, the amplitude of an evoked EPSC was measured every 20 seconds and when it was roughly the same for at least 3 consecutive EPSCs, usually about 4 minutes into the drug perfusion, the experiment could proceed. Spontaneous post-synaptic currents were recorded without application of these drugs.

\subsection{DATA ANALYSIS AND STATISTICS}

Evoked and spontaneous electrophysiological data were analyzed using custom procedures in IGOR Pro (Wavemetrics, USA).

Statistical analysis was performed using sPSs Statistics (IBM, USA) and Prism (GraphPad Software, USA). Statistical significance was determined using independent-samples $t$ test except where otherwise noted. Data are presented as mean and standard error of mean (SEM).

The non-negative tensor factorization analysis was performed in IGOR Pro using custom routines written by Prof. Dr. Dr. h.c. Erwin Neher.

2.5 RESOURCE LIST

Reagent or Resource Source IDENTIFIer

Chemicals

$\begin{array}{lll}\mathrm{NaCl} & \text { BioFroxx } & \text { I394 } \\ \mathrm{KCl} & \text { Sigma-Aldrich } & \text { P954I } \\ \mathrm{NaHCO}_{3} & \text { Roth } & 6885.2 \\ \mathrm{NaH}_{2} \mathrm{PO}_{4} & \text { Applichem } & \text { AI373.0500 }\end{array}$


Glucose

Ascorbic acid

Myo-inositol

Na-pyruvate

$\mathrm{MgCl} 2$

$\mathrm{CaCl} 2$

K-Gluconate

Hepes

TEA-Cl

Na2-Phosphocreatine

EGTA

Mg-ATP

Na2-GTP

CTZ

Kynurenic acid

IgorPro

SPSS Statistics

Prism
Applichem

Sigma-Aldrich

Sigma-Aldrich

Sigma-Aldrich

Honeywell

Sigma-Aldrich

Sigma-Aldrich

Sigma-Aldrich

Sigma-Aldrich

Sigma-Aldrich

Applichem

Sigma-Aldrich

Sigma-Aldrich

Sigma-Aldrich

Abcam

SOFTWARE

Wavemetrics

IBM

GraphPad
CAS\# 77938-63-7

A4544

I5I25

P2256

Mo250

C3306

$\mathrm{G} 4500$

$\mathrm{H}_{4} \mathrm{O} 34$

T2265

P7936

Ao878.0025

A9I87

G8877

C9847

AbI20256

Version 6.37

Version 2I

Version 7.04 
The first step in investigating Mover's role in synaptic transmission, was to establish the method of recording spontaneous and evoked excitatory postsynaptic currents (EPSCS) from MNTB principal cells in brainstem slices. MTNB cell bodies are the postsynaptic partners of the Calyx of Held and are ideal for patch-clamping them due to their relatively big size.

The voltage clamping of the postsynaptic neuron enables us to estimate the amount of neurotransmitter release via the measurement of the current flowing through the postsynaptic AMPA receptors. It is therefore an indirect method for studying the presynaptic mechanisms since the presynaptic currents can be shaped from the kinetics of the postsynaptic AMPA receptors.

Of particular interest are two properties of the AMPA receptors: saturation and desensitization. Receptor saturation refers to the binding at the postsynaptic receptors of the maximum amount of neurotransmitter molecules. When all the binding sites are occupied, any extra neurotransmitter that is being released has no effect at the postsynaptic side and therefore cannot be detected by measuring the postsynaptic current (Jonas et al., I993).

Desensitization is a kinetic property of the receptors, since they enter into a closed state rapidly and recover more slowly. When the neurotransmitter is present at the synapse for a longer time than the time course of the desensitization, then the receptors will be desensitized (Trussell et al., I993). At the calyx of Held, glutamate is binding to each of the four subunits of the AMPA receptor. Then, a conformational change is induced where the dimers formed between the four extracellular glutamate binding domains are rearranged and the channel opens. Desensitization is manifested as an additional rearrangement of these dimer interfaces such that the channel gate is decoupled from the domain closure. Therefore, although glutamate is bound to the dimmers, the channel is closed (Sun et al., 2002). 
AMPA receptor desensitization can be relieved, albeit incompletely, by the use of the compound cyclothiazide (CTZ) which stabilizes the receptor into a closed nondesensitized state, thereby preventing it into entering a desensitized conformation (Partin et al., I996). However, CTZ has also nonspecific presynaptic effects which render difficult the interpretation of physiological experiments. The most characteristic effect is the slowing of the decay time of the EPSC, which can result in some AMPA receptors not deactivating during the interstimulus interval (Meyer et al., 20or). Thus, temporal summation is more likely to occur and produce an apparent facilitation (Wong et al., 2003).

The first electrophysiological characterization of Mover was done in the Calyx of Held synapse of rats by Korber et al. (2015) using shRNA-mediated knockdown of Mover in bushy cells. Since there was no prior work done in the brainstem of knockout mice, I started the experiments by replicating as closely as possible the experimental conditions of Korber et al. so that my results could be directly comparable with the rat knockdown they used. For this reason, I used no pharmacological agents to reduce the desensitization and saturation of the postsynaptic AMPA receptors in the first set of experiments that I did. Nevertheless, it eventually became apparent that there were clear signs of AMPA-receptor desensitization.

In order to prevent AMPAR saturation and desensitization I proceeded with applying I mM kynurenic acid (KYN) and Ioo $\mu \mathrm{M}$ cyclothiazide (CTZ). This reduced saturation and desensitization of AMPARs, but did not prevent it entirely. Simultaneously, the application of CTz had the well-documented effect of lengthening the AMPAR opening time (Fucile et al., 2006). Thus the half-width of the EPSCS was increased. What was unexpected though, was that the opening times were being continuously increased throughout the course of cell recording.

To avoid this effect of CTZ, I resorted to using only KYN and in order to reduce to a minimum the AMPAR saturation and desensitization I doubled KYN's concentration to $2 \mathrm{mM}$. This setting proved to work well, as no signs of desensitization were visible anymore and the EPSCs continued to be well above the noise levels, even the steadystate level EPSCS of high-frequency stimulus trains. 
KYN is a low-affinity antagonist of glutamate that sequesters the majority of AMPA receptors into a blocked state; thus, the released ligand only has a small pool of receptors that can bind to and consequently deactivate, since the transition to the deactivated state requires binding of glutamate (Otis et al., 1996). During the time between two subsequent EPSCs, the fast kinetics of KYN allow a rapid re-equilibration between the blocked and unblocked receptors. Therefore, each glutamate event is detected by a different subset of AMPA receptors.

Others have successfully used KYN (I-2 $\mathrm{mM})$ to minimize receptor saturation in the calyx of Held during maximal glutamate release and have confirmed that transmission is not saturated since EPSC amplitude can be increased with greater extracellular calcium concentrations (Neher and Sakaba, 200I; Wong et al., 2003).

So, unless explicitly stated otherwise, all subsequent experiments have been done in the presence of $2 \mathrm{mM} \mathrm{KYN}$.

Additionally, like Korber et al., the mice I used were I2-I3 postnatal days old, at the period of hearing onset.

\section{I MOVER KO DOES NOT AFFECT BASIC SYNAPTIC TRANSMISSION}

I first started looking at Mover's role in synaptic transmission, by investigating basic synaptic transmission. By recording the spontaneous synaptic activity insights can be gained on the quantal size, the presynaptic release machinery and postsynaptic AMPAR kinetics. All recordings of spontaneous release have been done in the absence of KYN and CTZ, and only in slices where none of these drugs had been applied before.

If release is triggered by presynaptic action potentials then spontaneous EPSCs could be multiquantal. Nevertheless, when I $\mu$ M TTX was applied in the calyx of Held in order to block presynaptic APs, neither the amplitude nor the frequency of spontaneous EPSCS are affected (Ishikawa et al., 2002). Thus, spontaneous EPSCs in the calyx of Held, recorded without TTX, can also be considered true mini EPSCS (Schneggenburger and Forsythe, 2006).

No significant differences were found between wT and ko synapses in all of the properties measured (fig. 2). The amplitude of the spontaneous events can reflect the amount of glutamate per syn- 


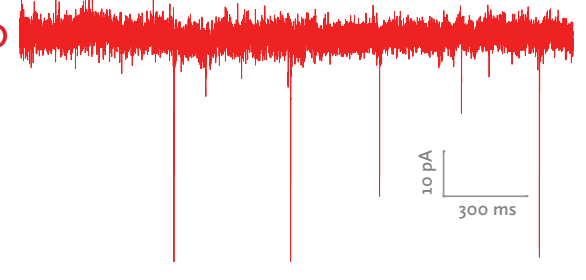

B
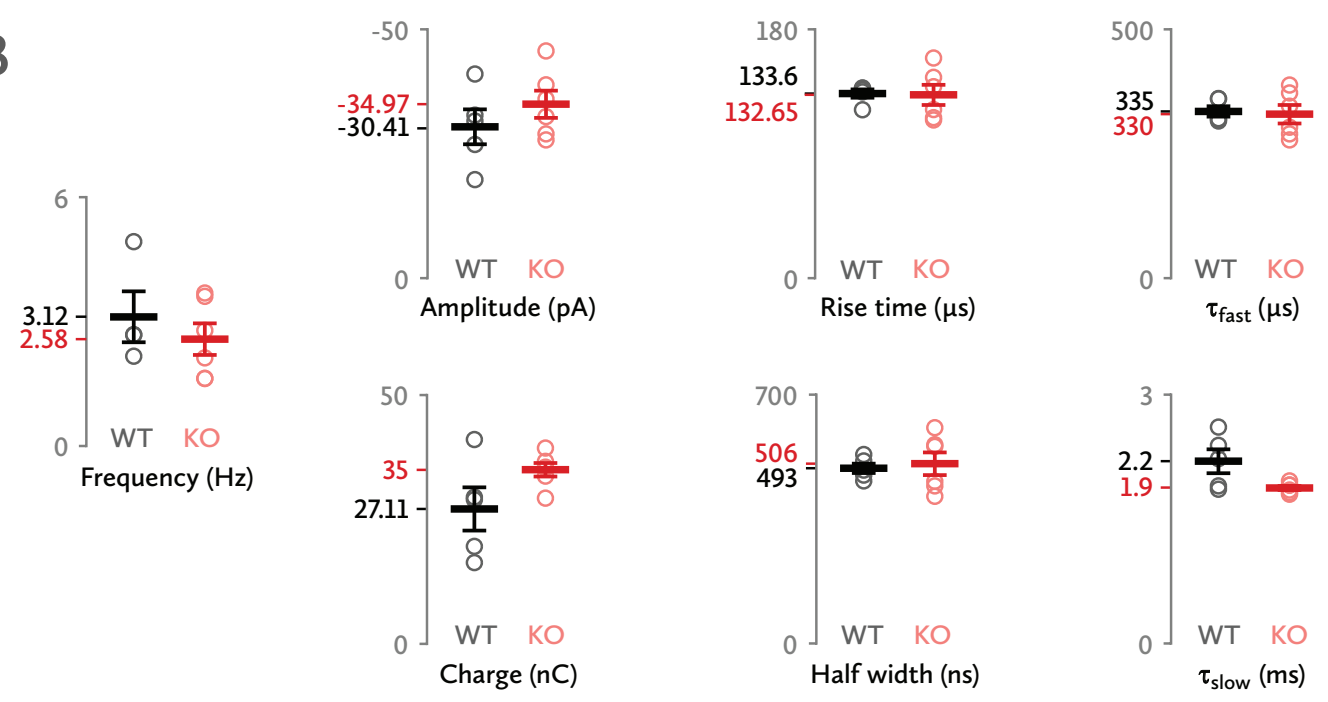

FIGURE 2: The basic release machinery and the postsynaptic AMPA-receptor kinetics are not altered by Mover. A: Representative current trace samples. B: Frequency, amplitude, and kinetics of spontaneous release.

aptic vesicle, the number of postsynaptic AMPARs as well as the single-channel conductance, and it was unchanged in the Ko (wild type: $-30.4 \mathrm{I} \pm 3.5 \mathrm{nA}, n=5$; knock out: $-34.97 \pm 2.73 \mathrm{nA}, n=6$; independent-samples t-test; $\left.t_{(9)}=1.042 ; p=0.325\right)$.

On the other hand, the frequency of the events can point to synaptic-vesicle release probability dependent on active-zone numbers and the number of docked svs per active zone. No differences were found between WT and KO in the frequency of spEPSCs (wild type: $3.12 \pm 0.62$ $\mathrm{Hz}, n=4$; knock out: $2.58 \pm 0.38 \mathrm{~Hz}, n=6$; independent-samples t-test; $\left.t_{(8)}=0.792 ; p=0.45 \mathrm{I}\right)$.

Finally, the spEPsc kinetics, such as half width, rise time and time constants of decay can indicate changes in the postsynaptic single-channel kinetics, but they were the same between wT and ко (half width; wild type: $493.05 \pm 13.37 \mathrm{~ns}, n=5$; knock out: $506 \pm 31.72$ ns, $n=6$; independent-samples t-test; $t_{(6.67)}=-0.375 ; p=0.719$; rise 
time; wild type: $\mathrm{I} 33.6 \pm 2.97 \mu \mathrm{s}, n=5$; knock out: $132.65 \pm 7.35 \mu \mathrm{s}, n=6$; independent-samples t-test; $t_{(6.547)}=0.12 ; p=0.908$; charge; wild type: 27.II $\pm 4.34 \mathrm{fC}, n=5$; knock out: $35 \pm \mathrm{I} .37 \mathrm{fC}, n=6$; independent-samples t-test; $t_{(9)}=-$-I.878; $p=0.093$; fast decay time constant; wild type: 335.4I \pm I0.44 $\mu \mathrm{s}, n=5$; knock out: $329.63 \pm$ I8.66 $\mu \mathrm{s}, n=6$; independent-samples t-test; $t_{(7.68)}=0.255 ; p=0.794$; slow decay time constant; wild type: $2.2 \pm 0.14 \mathrm{~ms}, n=5$; knock out: $\mathrm{I} .88 \pm 0.25 \mathrm{~ms}, n=6$; independent-samples t-test; $t_{(4.239)}=2.225 ; p=0.086$ ).

Since the spontaneous SV release properties are similar between WT and ко, we can assume that the basic release machinery, the number of active zones and the postsynaptic AMPAR kinetics are not affected by Mover.

\subsection{MOVER KO DOES NOT AFFECT EVOKED RELEASE}

To investigate Mover's effect on phasic $\mathrm{Ca}^{2+}$-induced release, the afferent fibers were electrically stimulated; thereby single presynaptic action potentials were elicited and the resulting EPSCs were recorded from the postsynaptic principal cell. Every cell was stimulated io times, once every Io seconds and the resulting EPSCs were first offset corrected, then aligned based on their half-rise levels and finally averaged in order to measure the EPSC properties described below.

Although there was a trend for smaller EPSC amplitudes in the Ko, mainly due to the absence of high-initial-amplitude synapses, there was not a statistically significant difference (fig. 3) (wild type: $-0.96 \pm 0.098 \mathrm{nA}, n=\mathrm{I} 7$; knock out: $-0.83 \pm 0.058 \mathrm{nA}, n=\mathrm{I} 9$; independent-samples t-test; $t_{(34)}=\mathrm{I} .2 ; p=0.238$ ).

A measure of the synchronicity of the synaptic-vesicle fusion with the presynaptic membrane upon the arrival of an action potential is the rise time of the EPSC. In both wT and кO rise time was at the same levels (wild type: I79.92 $\pm 4 . \mathrm{I} 22 \mu \mathrm{s}, n=\mathrm{I} 3$; knock out: I84.56 $\pm 2.756 \mu \mathrm{s}, n=9$; independent-samples t-test; $t_{(20)}=-0.85$; $p=0.407)$. The rest of the EPSC kinetics such as the half width, total charge and decay time constants were also unchanged (half width; wild type: $701.09 \pm 42.053 \mu \mathrm{s}, n=\mathrm{I} 3$; knock out: $750.64 \pm 25.822 \mu \mathrm{s}$, $n=9$; independent-samples t-test; $t_{(20)}=-0.9 ; p=0.380$; charge; wild type: I. $32 \pm 0.185 \mathrm{pC}, n=\mathrm{I} 3$; knock out: I. $44 \pm 0 . \mathrm{II} 6 \mu \mathrm{s}, n=9$; independent-samples t-test; $t_{(\mathrm{r} 8.88)}=-0.53 ; p=0.60 \mathrm{o}$; fast decay time constant; 

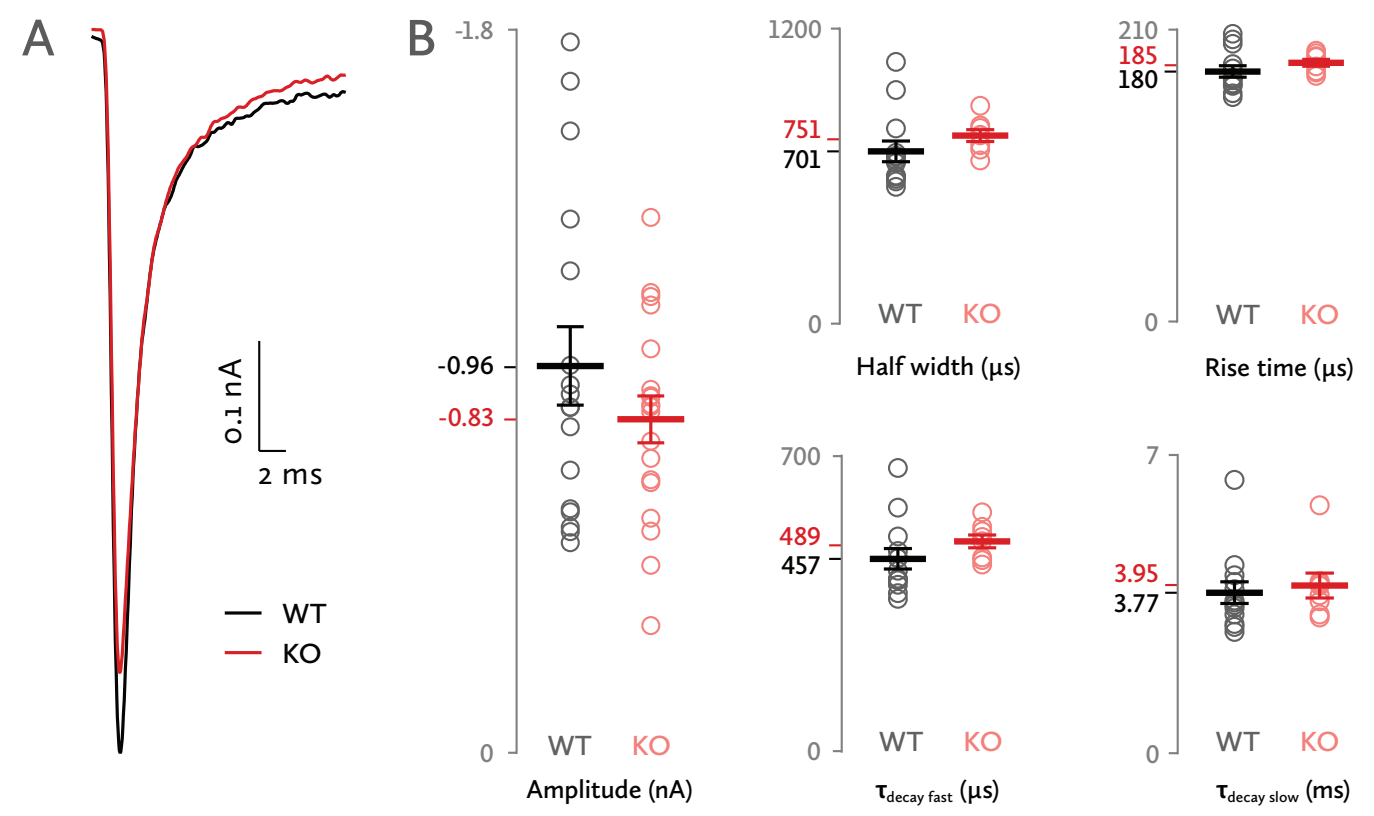

FIGURE 3: Evoked EPSCs do not differ between WT and KO calyxes, except for a slight reduction in amplitude in the KO synapses. A: representative traces $\mathrm{B}$ : amplitude and kinetics of evoked EPSCs

wild type: $456.52 \pm 24.26 \mathrm{I} \mu \mathrm{s}, n=\mathrm{I} 3$; knock out: $488.79 \pm \mathrm{I} 6.39 \mu \mathrm{s}, n=9$; independent-samples t-test; $t_{(20)}=\mathrm{I} ; p=0.33$; slow decay time constant; wild type: $3.77 \pm 0.256 \mathrm{~ms}, n=\mathrm{I} 3$; knock out: $3.95 \pm 0.258 \mathrm{~ms}$, $n=9$; independent-samples t-test; $\left.t_{(20)}=0.47 ; p=0.645\right)$.

\subsection{INITIAL RELEASE PROBABILITY IS DECREASED IN THE MOVER KO}

\subsection{Paired-pulse ratio is increased in the KO synapses}

Changes in the initial release probability can be reflected in a simple measurement called paired-pulse ratio. It is produced by two closely-timed stimuli, which result in two EPSCS; the ratio of the second EPSC over the first is the paired-pulse ratio. As outlined in the introduction, the basic depletion model (Zucker and Regehr, 2002) can interpret the paired-pulse depression observed in the calyx of Held synapse (Scheuss et al., 2002). As such, a low initial release probability results in a high paired-pulse ratio (PPR).

In order to have a basic assessment of changes of the initial release probability, I tested WT and Ko synapses in a variety of paired-pulse 

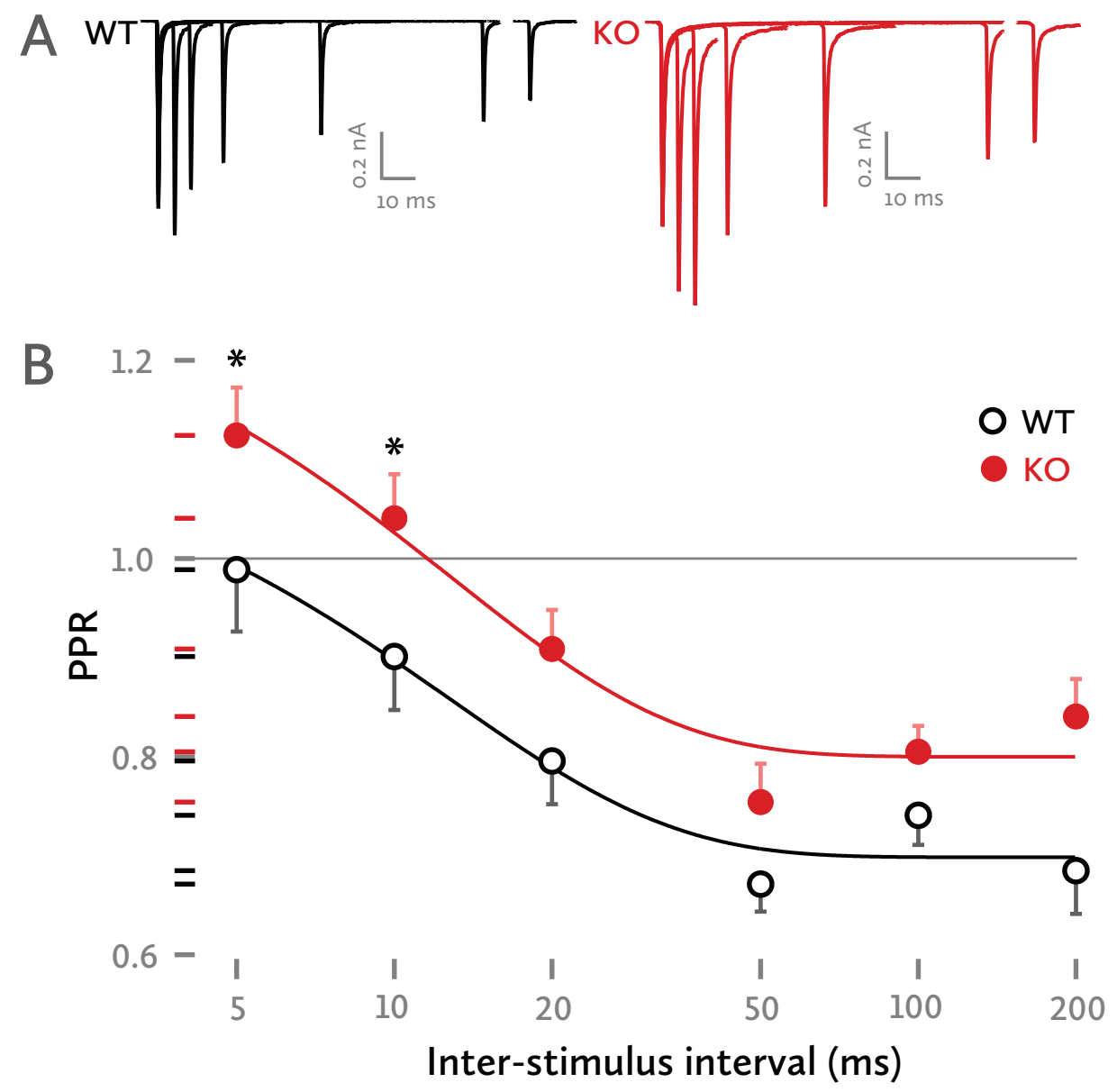

FIGURE 4: KO synapses have a consistently higher paired-pulse ratio. A: Representative traces from WT and KO calyces. B: PPRs in various inter-pulse intervals. The PPR in 5- and 10-ms inter-pulse intervals was significantly higher in $\mathrm{KO}$ animals.

intervals, ranging from $5 \mathrm{~ms}$ to $200 \mathrm{~ms}$. The ko had a consistently higher PPR than the WT as indicated by a linear mixed-model analysis of the two groups (fig. 4) (wild type: $0.797 \pm 0.022$; knock out; $\left.0.9 \mathrm{I} 2 \pm 0.02 ; F_{(\mathrm{I}, \mathrm{I} 38)}=\mathrm{I} 5 . \mathrm{I} 72, p<0.000\right)$.

Post-hoc tests revealed statistically significant differences at 5- and Io-ms inter-pulse intervals $(5 \mathrm{~ms}$; wild type: $0.989 \pm 0.04 \mathrm{I}$, $n=\mathrm{I} 7$; knock out; I.I24 $\pm 0.039, n=\mathrm{I9} ; p=0.019$; Io ms; wild type: $0.90 \mathrm{I} \pm 0.044, n=\mathrm{I} 5 ;$ knock out; I.04I $\pm 0.044, n=\mathrm{I} 5 ; p=0.027$ ). Thus, we can conclude that in Mover's absence from the Calyx of Held synapse there is a trend towards a reduction of the initial release probability. 


\subsubsection{High-frequency stimulation-train time constant}

but not extent of depression is increased in the ко

Based on the previous findings, this research was continued in the direction of release probability. For that reason, I stimulated the axons with 50-pulse trains of various frequencies, starting from $5 \mathrm{~Hz}$ and reaching up to Ioo $\mathrm{Hz}$. Then the EPSC amplitudes were plotted versus the stimulus number and these data points were fitted with a mono-exponential curve. In the low-strength frequencies of 5 , Io and $20 \mathrm{~Hz}$, there was no difference in the decay constant $(\mathrm{tau}-\tau)$ between WT and Ko animals for each frequency. Akaike's Information Criteria were used to select the model that was most likely to have generated the data $(20-\mathrm{Hz}$ trains; common $\tau=3.06 \pm 0.46$, wild type $n=7$, knock out $n=8$; Io-Hz trains; common $\tau=2.49 \pm 0.38$, wild type $n=\mathrm{I} 3$, knock out $n=\mathrm{I} 3 ; 5-\mathrm{Hz}$ trains; common $\tau=2 . \mathrm{I} 2 \pm 0.38$, wild type $n=5$, knock out $n=7$; all datasets were fit from the 2 nd until the 5oth pulse).

However at the frequencies of 50 and $100 \mathrm{~Hz}$ the time course of the KO synapses was significantly slower, as seen in figure $5(50-\mathrm{Hz}$ trains; wild type; $\tau=2.78 \pm 0.38, n=\mathrm{I} 4$; knock out; $\tau=3.47 \pm 0.46$, $n=$ I3; IOO-Hz trains; wild type; $\tau=3.38 \pm 0.33, n=$ I5; knock out; $\tau=4.24 \pm 0.4 \mathrm{I}, n=\mathrm{I} 5$; all datasets were fit from the 2 nd until the 5oth pulse).

Short-term depression at the calyx of Held synapse in frequencies up to $30 \mathrm{~Hz}$ is thought to be mediated mostly through a $\mathrm{Ca}^{2+}$-induced inhibition of presynaptic calcium channels whereas depression from Ioo $\mathrm{Hz}$ and higher is mainly due to depletion; at intermediate frequencies, both mechanisms are contributing ( $\mathrm{Xu}$ and $\mathrm{Wu}, 2005)$. Based on that, the present results which show a statistically significant slower time course of the Ko synapses only at 50 and $100 \mathrm{~Hz}$, could indicate a reduced release probability in the ko calyxes that is mostly due to depletion and not due to a decrease in the presynaptic calcium current.

Additionally, the steady-state levels, as determined by the amplitudes of the last 20 EPSCS of each train, were not significantly different (fig. 5 B). For a formal comparison between steady states of WT and Ko calyces, a two-way mixed ANOva was employed (Io Hz; wild type: $0.2 \mathrm{I} \pm 0.03 \mathrm{nA}, n=\mathrm{I} 3$; knock out: $0.27 \pm 0.03 \mathrm{nA}, n=\mathrm{I} 3$; 

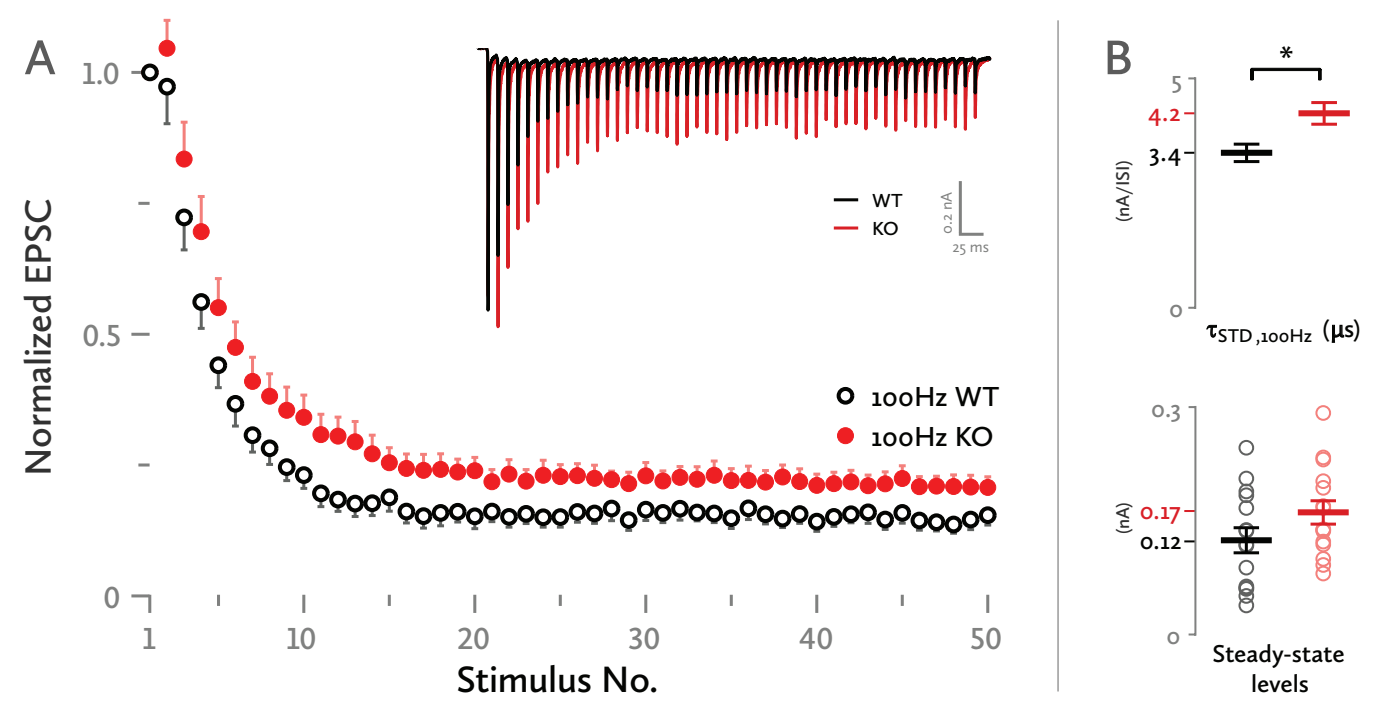

FIGURE 5: The KO synapses reach the same steady-state levels more slowly. A: Normalized plot of EPSCs during a 100-Hz stimulation train. Inset: Representative traces from WT and KO animals. B: The time constant and the steady-state levels of WT and KO animals.

$F_{(\mathrm{I}, 25)}=2.3 \mathrm{I} 9 ; p=0.14 ; 50 \mathrm{~Hz}$; wild type: $0.16 \pm 0.02 \mathrm{nA}, n=\mathrm{I} 4$; knock out: $0.2 \mathrm{I} \pm 0.02 \mathrm{nA}, n=\mathrm{I} 3 ; F_{(\mathrm{I}, 26)}=2.014 ; p=0.168 ;$ I0o Hz; wild type: $0.12 \pm 0.02 \mathrm{nA}, n=\mathrm{I} 5$; knock out: $0.17 \pm 0.02 \mathrm{nA}, n=\mathrm{I} 5 ; F_{(\mathrm{I}, 29)}=3.573$; $p=0.069)$.

Since the steady state is thought to be supported entirely by newly-recruited svs, the unaffected amplitude of the steady-state EPSCs suggests that in both WT and KO synapses there is the same number of svs being recruited during the train.

\subsubsection{Readily-releasable pool size}

An estimation of the size of the readily-releasable pool and its replenishment rate can be acquired by fitting a straight line at the linear part of a cumulative EPSC vs. stimulus number graph, induced by a IOO-Hz train; The $y$-axis intersection of this straight line is the RRP and its slope is the replenishment rate of the RRP (fig. 6 A) (Schneggenburger et al., I999). Both of these factors were unchanged in the KO calyces (fig. $6 \mathrm{~B}$ ) (RRP; wild type: $3.55 \pm 0.33 \mathrm{nA}, n=\mathrm{I}$; knock out: $3.93 \pm 0.34 \mathrm{nA}, n=\mathrm{I} 5 ; t_{(28)}=-0.806 ; p=0.427$; replenishment rate; 

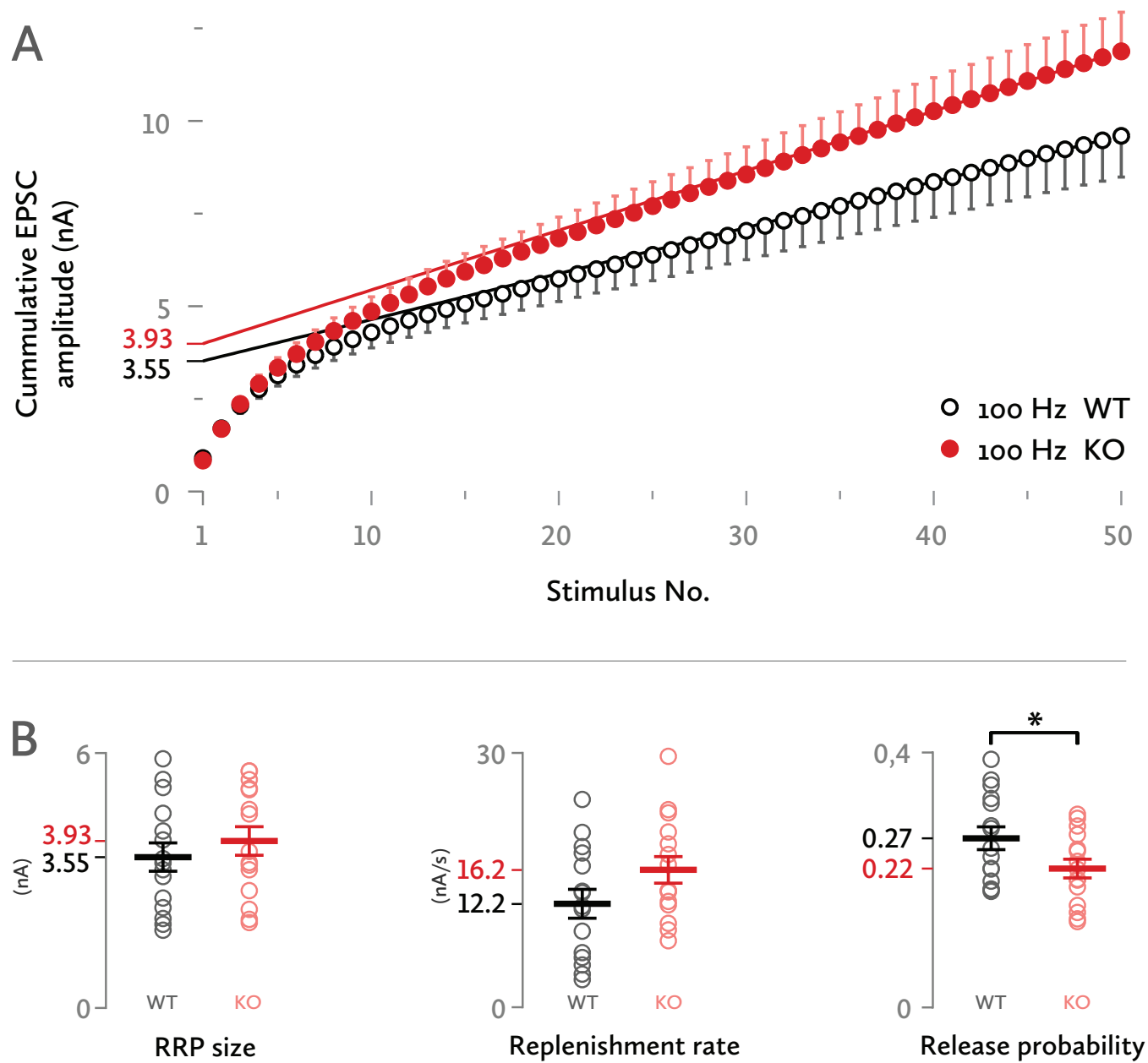

FIGURE 6: The release probability of the KO calyces is smaller. A: Cumulative plot of the $100-\mathrm{Hz}$ trains. A straight line was fit to the 10 last data points for each curve and then extended until it intersected the $y$ axis. B: Measurements of the readily-releasable pool, the replenishment rate and the release probability.

wild type: $\mathrm{I} 2.2 \pm \mathrm{I} .7 \mathrm{nA} / \mathrm{s}, n=\mathrm{I} 5$; knock out: $\mathrm{I} 6.2 \pm \mathrm{I} .6 \mathrm{nA} / \mathrm{s}, n=\mathrm{I} 5$; $\left.t_{(28)}=-\mathrm{I} .727 ; p=0.095\right)$.

Since the size of the RRP is known, the initial release probability can be calculated by dividing the EPSC $\mathrm{I}_{\mathrm{I}}$ amplitude over the RRP size. Release probability at the KO synapses was significantly lower than at the WT (fig. $6 \mathrm{~B}$ ) (wild type: $0.266 \pm 0.018, n=\mathrm{I} 5$; knock out: $\left.0.2 \mathrm{I} 8 \pm 0.015, n=\mathrm{I} 5 ; t_{(28)}=2.048 ; p=0.05\right)$. The lower release probability of the KO svs was due to the combination of two factors: slightly smaller EPSC $\mathrm{I}_{\mathrm{I}}$ amplitudes as seen in fig. 3 and a marginally bigger RRP size. The quotient of these two measurements, although they did not differ significantly when compared individually, produced a third 

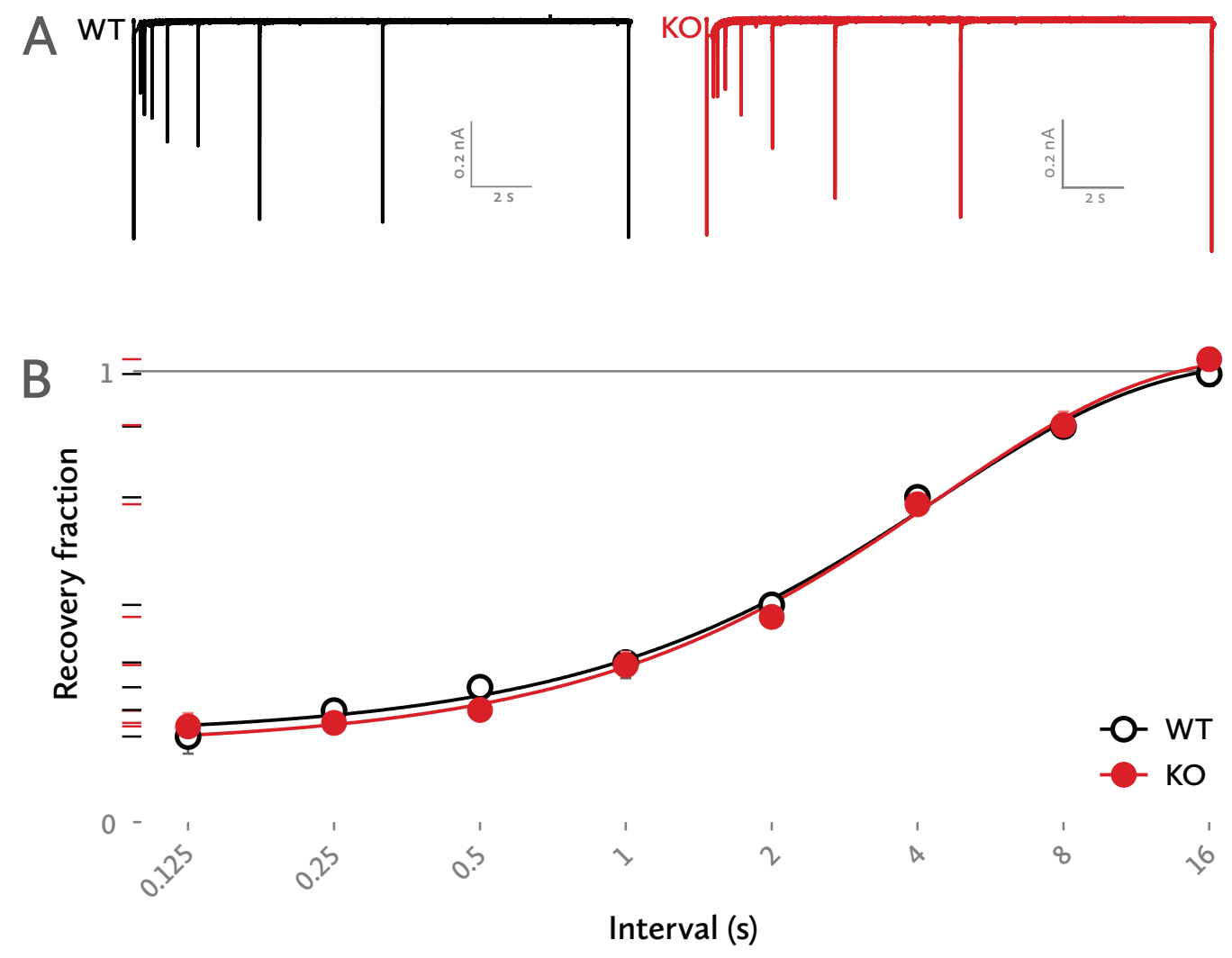

FIGURE 7: Recovery from depression is unaffected. A: Representative traces from WT and KO synapses. B: The recovery fraction for each interval from a depletion train. Each interval was studied with a separate depletion train. In this way, each data point is independent from interactions with the previous ones. The $x$-axis scale is logarithmic to clearly display the initial short time intervals.

measurement, the release probability, that was statistically different between WT and ko synapses.

\subsection{RECOVERY FROM SYNAPTIC DEPRESSION IS UNAFFECTED}

To further probe whether the replenishment rate was affected by the absence of Mover, the recovery from depression was measured. More specifically synaptic depression was induced in the calyx of Held by 20 stimuli at roo $\mathrm{Hz}$. Then, at various intervals after the end of the stimulation train a single EPSC was elicited (fig. 7 A). The ratio of its amplitude compared with the amplitude of the first EPSC of the train is plotted in figure $7 \mathrm{~B}$. 
An exponential reaching a plateau was fit to the data and their growth constant $\mathrm{k}$ was compared, according to Akaike's information criteria (AICC). No differences were found between wT and Ko synapses (wild type; $n=\mathrm{I} 2$; knock out; $n=\mathrm{II}$; common k: $0.23 \pm 0.03$; $\mathrm{R}^{2}$ : 0.997). This indicates that the reloading of the RRP after synaptic depression is unchanged.

\subsection{THE SUPERPRIMED SV POOL IS AFFECTED IN MOVER KO}

So far, the data provide multiple indications of a reduced released probability in the KO synapses. The traditional models of calculating the release probability regard the RRP as a homogeneous population of vesicles, ready to fuse with the presynaptic membrane at the arrival of an action potential.

However, as detailed in the introduction, there are strong indications that the population of primed synaptic vesicles is heterogeneous and a model that takes into account these indications is the one proposed by Taschenberger et al. (2016). There, the authors are describing two states of primed vesicles; a quickly-replenished and low release-probability state (normally-primed svs), and a slowly-replenished state but with a high release probability upon the arrival of an action potential (superprimed svs).

The calyx of Held synapses show a well-documented heterogeneity in their synaptic strength when they are at rest. However, this variance disappears during repetitive high-frequency stimulation in WT cells. In fig. 8 A there is an example of two wT cells: one with a high initial EPSC amplitude (EPSC $)$ and one with a low EPSC ${ }_{\mathrm{I}}$. Both

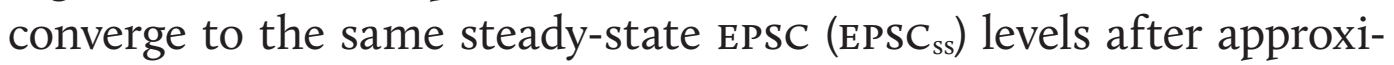
mately io stimulations at $200 \mathrm{~Hz}$. The model postulates that this initial heterogeneity results from different levels of superprimed svs in the synapses and since this group of vesicles is rapidly depleted and not sufficiently replenished during a high-frequency train, the lowbut steady-amplitude EPSCs observed at the late stages of a stimulus train are contributed mostly by normally-primed svs. Consequently, the steady-state levels of a high-frequency train are independent of the initial EPSC levels. However, in less intense frequencies such as 50 or Io $\mathrm{Hz}$, there is a stronger correlation between the initial EPSC and the steady-state levels. According to the model, this happens be- 
cause between two stimuli there is more time for superpriming of new synaptic vesicles, the rate of which varies between cells.

If we plot every wT cell's initial EPSC versus the steady-state EPSC for the $200-\mathrm{Hz}$ trains (purple dashed line in fig. $8 \mathrm{C}$ ), we see that they are almost parallel to the $x$ axis, reflecting the similar steadystate levels they reach in such a strong stimulation. Conversely, if we plot the same wT cells when stimulated at Io $\mathrm{Hz}$ (blue dashed line in
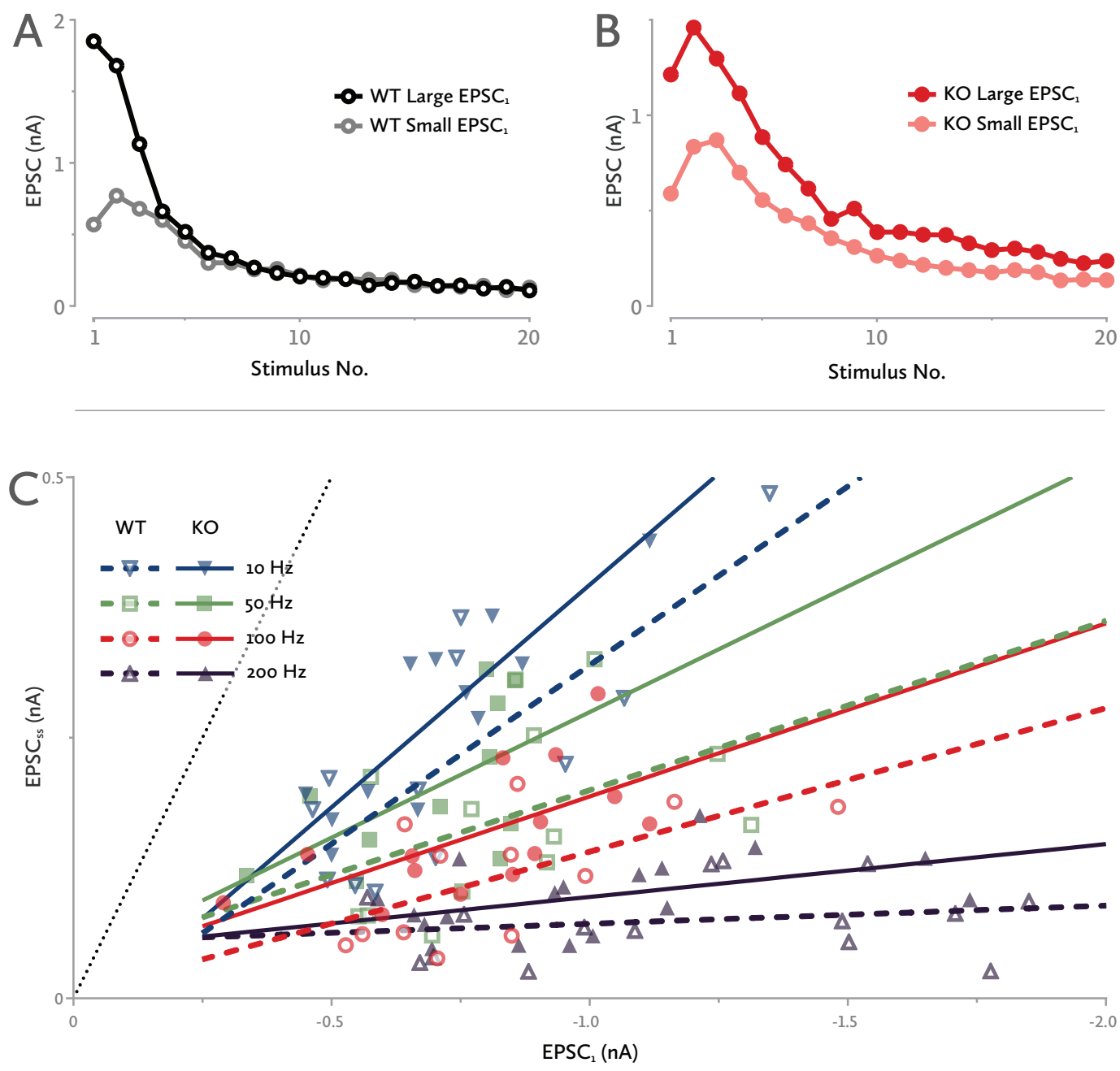

FIGURE 8: The superprimed component of the KO synapses gets depressed less than the WT ones. A: Representative examples of two WT cells stimulated at 200 $\mathrm{Hz}$; one with a high initial EPSC amplitude and one with a low amplitude. Both converge at the same steady-state levels. B: KO synapses retain their initial amplitude differences even at the steady state during a high-frequency stimulation. C: The depression exhibited during a stimulation train is dependent on the frequency used and on the initial EPSC amplitude of each synapse. 
fig. $8 \mathrm{C}$ ) we see that the correlation between $\mathrm{EPSC}_{\mathrm{I}}$ and $\mathrm{EPSC}_{\mathrm{ss}}$ is much stronger, mirroring to a high degree the differences in EPSC ${ }_{\mathrm{I}}$. Accordingly, the higher the train frequency, the weaker the correlation, as seen in the rest of the dashed lines in fig. $8 \mathrm{C}$. In this graph, every open data point corresponds to a WT cell at a specific stimulation frequency, and the dashed lines represent a linear regression fit to the wT cells.

The slope $(\Delta y / \Delta x)$ of the regression lines depends only on the superprimed svs, thus the slope of a given frequency mainly reports the depression that the superprimed component undergoes throughout the train.

Performing the same kind of analysis on the ko synapses, we see a marked difference; the correlation between $\mathrm{EPSC}_{\mathrm{I}}$ and $\mathrm{EPSC}_{\mathrm{ss}}$ is stronger in every frequency, and is even present at $200-\mathrm{Hz}$ trains. In fig. 8 $\mathrm{B}$ two representative Ko cells can be seen, one with a high and one

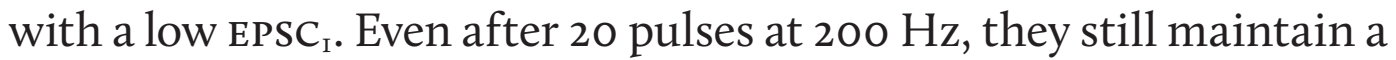
small degree of their initial differences in EPSC amplitude. The same holds true, to a greater extent than the WT, for lower frequencies like Io Hz. If we plot the ko cells the same way as the wT cells before, we can observe that the slopes of the line fits for the Ko synapses are consistently higher than the respective ones for the wT synapses (fig. 8 C). This indicates a smaller short-term depression of the superprimed component in Ko calyces.

All the line fits, both for WT and ko calyces, converge at the same point in the $x$ axis, around $-0.25 \mathrm{nA}$. The model assumes that an EPSC is the contribution of normally-primed and superprimed svs and that only the superprimed component varies from synapse to

FIGURE 9 (OPPOSITE PAGE): Mover is increasing the release probability of the superprimed SVs only. A: Isolation of the superprimed component of the calyces of Held. The time constant of an exponential fit to the data points is larger for the KO synapses, indicating a lower superprimed SV release probability for that group. Similarly, the $y$-axis intersection of a straight line fit to the last data points of the cumulative plot, indicates also a lower release probability for the superprimed SVs of the KO cells. The small bump in the cumulative plot between stimulus numbers 5 and 15 is an indication of postsynaptic receptor desensitization, mostly at the

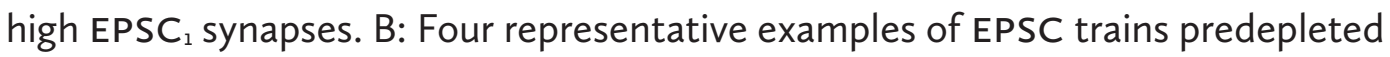
by a low-frequency train. 
synapse. Thus, we can conclude that the contribution of the normally-primed svs in every calyx is $-0.25 \mathrm{nA}$. The rest of the current for every synapse's $\mathrm{EPSC}_{\mathrm{I}}$ comes from superprimed svs; the more they contribute, the higher this cell's data point moves along the line fit.

The next step was to try to isolate the superprimed sv contribution during a stimulus train. As was mentioned before, the model assumes that the normally-primed component is relatively invariant
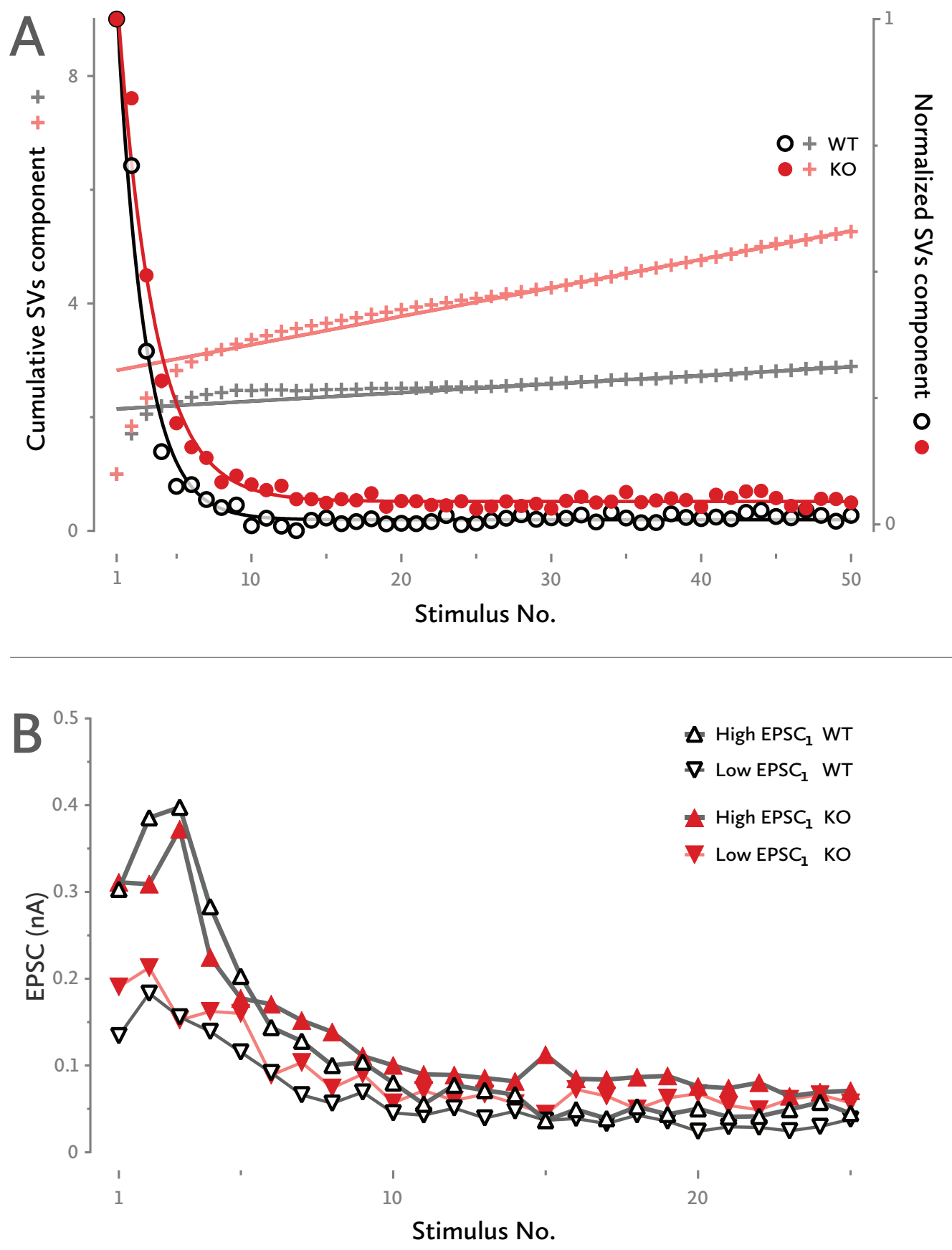
among synapses, whereas the superprimed component varies. Consequently there can be synapses with a small superprimed component and others with a large one, but all of them will have a normally-primed component of the same size. The second assumption is that the normally-primed component stays stable throughout a stimulus train, while the superprimed component is rapidly depleted during a high-frequency train. Based on that, if we find the group of cells with the lowest EPSC $_{\mathrm{I}}$ amongst the total pool of cells recorded, then we have a group of synapses with a very small superprimed component. If we consequently subtract these low $\mathrm{EPSC}_{\mathrm{I}}$ cells from another group of synapses with the highest $\mathrm{EPSC}_{\mathrm{I}}$ - and thus with the biggest superprimed component - then we are essentially removing from the high-EPSC ${ }_{\mathrm{I}}$ cells only the normally-primed component, leaving almost intact the superprimed one.

However, one could argue that the normally-primed component may not be entirely uniform among synapses and small variations could exist. The authors propose a simple but well-designed solution to compensate for this variance; since the slope of the regression line of fig. $8 \mathrm{C}$ reports the depression of the superprimed component, then the normalized average steady state of the small EPSC $_{\mathrm{I}}$ group of cells should have the same amount of depression. In the case of WT calyces, the slope of the $200-\mathrm{Hz}$ line was 0.0174 which meant that the cells with a small EPSC $_{\mathrm{I}}$ were multiplied by a factor of $\mathrm{I} .365$ to achieve the same depression at steady state ( (mall-EPSC $_{\mathrm{I}}$ cells; $n=3$; large-EPSC $\mathrm{I}_{\mathrm{I}}$ cells; $n=4$ ). Accordingly, the KO small $\mathrm{EPSC}_{\mathrm{I}}$ calyces were multiplied by 0.89 to achieve a depression of 0.05075 , calculated from the KO 200-Hz regression line of fig. $8 \mathrm{C}$ (small-EPSC I $_{\mathrm{I}}$ cells; $n=3$; large-EPSC cells; $n=5$ ). The resulting graphs are plotted in fig. 9 A.

Since these traces are formed by subtraction of one group of cells from another, they cannot provide estimates about the absolute superprimed pool sizes of individual synapses. Thus, they are normalized. However, it is possible to estimate the superprimed release probability $\left(p_{s}\right)$ by using the back-extrapolation method used previously as well, by dividing the initial EPSC value of I with the $y$-axis intersection of a straight line fitted to the last Io EPSCS of a cumulative plot (fig. 9 A). For the wT calyces the $p_{s}$ is $0.47 \pm 0.008$, and for the KO calyces is $0.35 \pm 0.004$, indicating a significantly reduced release probability for the superprimed synaptic vesicles in the kо synapses 
(AICs indicated that the Y intercept was $99.99 \%$ probable to be different for each data set).

All synapses converge at the same steady-state levels, independently of whether they are WT or Ko, or having a high-amplitude $\mathrm{EPSC}_{\mathrm{I}}$ or a low-amplitude one.

According to the authors' model there is another way to calculate the $p_{s}$ from the superprimed component's decay course. The inverse of the time constant of an exponential fit is equal to the sum of the $p_{s}$ and the priming rate constant. However, the priming rate of superprimed svs at $200 \mathrm{~Hz}$ is close to zero. Therefore the inverse of the time constant can be considered almost equal to $p_{s}$. For the wT cells the $p_{s}$ amounted to $0.55 \pm 0.02$ whereas at the kO cells it was $0.4 \mathrm{I} \pm 0.02$. Thus, the ko calyces are again found to have a significantly smaller ps (AICs indicated that the decay constant was $99.99 \%$ probable to be different when comparing the two data sets). The slightly increased $p_{s}$ values that the exponential-fit method reported could be due to facilitation during the stimulus train; the exponential fit is largely influenced by the steady-state levels, whereas the cumulative fit is mostly affected by EPSC . $_{\text {. }}$

$\mathrm{SV}_{\mathrm{s}} \mathrm{s}$ become depressed even during a IO-Hz train, as evidenced from figure $8 \mathrm{C}$, leaving less than $20 \%$ of the superprimed pool intact (Taschenberger et al., 20I6). Furthermore, the time course of the superprimed component shown in fig. 9 A shows that the svs pool rapidly depletes during a stimulation train. Based on this, the time course and the properties of the normally-primed pool can be studied without interference from superprimed vesicles.

If a Io-stimuli Io- $\mathrm{Hz}$ train is used before a Ioo- $\mathrm{Hz}$ stimulus train, then we expect to have a greatly depleted superprimed pool at the beginning of the Ioo- $\mathrm{Hz}$ train. Then, the short-term dynamics of the normally-primed sv pool are going to dominate the pre-depleted high-frequency train. Indeed, fig. 9 B shows two such examples, for each animal group, wT and KO. It is apparent that the time course of the EPSCs is similar irrespective of whether the cell had a low or a high EPSC $_{1}$; after a few stimulations the EPSCs converge. The same holds true for the ko synapses as well. From the graph is also apparent that the short-term depression normally exhibited by naïve synapses is now converted into a pronounced initial facilitation followed by depression. 
As detailed earlier, the inverse of the time constant of an exponential fit to the data equals with the sum of the normally-primed release probability and the priming rate constant. In contrast with the svs component, the priming rate of the svn component is not negligible and must be taken into account. Hence, only a range of $p_{n}$ can be reported. Both wT and ko normally-primed synaptic vesicles lie in the same range of release probabilities $\left(p_{n}<0.15\right.$, AICs indicated that the decay constant was $59.6 \%$ probable to be the same for both data sets).

Therefore, Mover is increasing the release probability only of a specific subset of docked and primed synaptic vesicles: the ones which are ready to be released first upon the arrival of an AP at a rested synapse.

\subsection{LOWER RELEASE PROBABILITY OF THE KO TIGHT-STATE COMPONENT}

\subsection{Non-negative tensor factorization theory}

As described in the introduction, there is increasing evidence pointing away from a uniform pool of release-competent svs. Indeed, most studies point to the existence of functionally-different svs that dock to a static number of release sites. More specifically, the proposed model by Neher \& Brose (2018) suggests the existence of two different sv docking states; a loose state (LS) and a tight state (TS). The exocytosis of sv contents occurs predominantly from the tightly-docked pool and the transition from LS to TS is strongly dependent on the intracellular local $\mathrm{Ca}^{2+}$ concentration.

Therefore, an EPSC train in a synapse that shows strong depression, like the calyx of Held, can be analyzed into three different overlapping components: a TS component, an LS component and a newly-recruited (RS) component, the later one being comprised of svs that are docked, primed and released during the stimulation train. Ordinary methods for estimating the size of the synaptic vesicle pools do not distinguish between the loose- and the tight-state sv pools (Neher, 2015), since the transition from one state to the other is hypothesized to occur at the ms scale. Thus, the problem arises on how to separate a mixed signal, the EPSC train, into its constitutive components without being able to directly observe them separately. 
This problem can be paraphrased using a real-world example. There is a group of three people engaged in a heated discussion. They frequently interrupt each other and at many instances they are all talking at the same time. The only record we have from this discussion is from a microphone placed somewhere in the room. The question is how we can separate this mixed recording into three separate recordings, one for every person.

One effective method to decompose a signal into its components is by using a technique called blind source separation. It works by separating a mixed signal into its source signals by only using, if at all, some inferred knowledge like statistical independence.

In our case, the source signals are non-negative because they represent currents flowing into one direction; this is a small but important bit of information as it can greatly restrict the possible solutions of the algorithm. Another important information that we can use to decompose the mixed signal is the predetermined dimensionality of the recordings; we have a fixed number of stimuli per train, a specific combination of trains and the same stimulation routine run through every cell of the dataset.

Although we cannot directly observe the separate components, we can experimentally remove a big fraction of the Ts component from an EPSC train. As detailed in section 3.5, a protocol that includes some conditioning pulses at a lower frequency can effectively deplete the superprimed, or in this model, the Ts pool. Here, I have used a modest predepletion protocol comprising of 2 pulses at Io $\mathrm{Hz}$ just before the normal high-frequency train. Therefore, for a given frequency I stimulated each cell both with a "straight" train and with a train preceded by the conditioning pulses.

It can be assumed that the recorded EPSC at cell $x$, using the predepletion protocol $s$ (with either o or 2 predepletion pulses), at the stimulus number $y$ is the sum of the three components: TS, LS and RS component. The impact of a component $c$ is proportional to its pool size $\alpha$ at cell $x$, its base function $s$ at stimulus number $y$ and its attenuation factor $q$ at the predepleted protocol $s$. It can be summarized with the equation

$$
e_{x y s}=\sum_{c=1}^{3} a_{c x} s_{c y} q_{c s}
$$


By amplitude I refer to the total amount of svs dedicated to each component over the course of a train; the base function refers to time course of the component; the attenuation factor quantifies the weakening of each component when using a predepleted stimulation protocol.

If $a_{c x}, s_{c y}$ and $q_{c s}$ are regarded as matrices and $e_{x y s}$ as a three-dimensional tensor then the above equation can be written as

$$
\underline{E}=A S Q
$$

where every one of the resulting matrices can provide valuable information. Since all three of them are unknown, the non-negative tensor factorization (NTF) method can be employed to estimate them.

Tensors can be considered as multi-dimensional matrices. The order of the tensor is the number of dimensions - in our case it is three. A zero-order tensor is a scalar, a first-order tensor is a vector, a second-order tensor is a matrix, and third- and higher-order tensors are simply called higher-order tensors. A three-dimensional tensor is shown in figure Io.

In order to describe how the NTF works, one step back will be taken in order to simplify the problem. Consider that instead of a

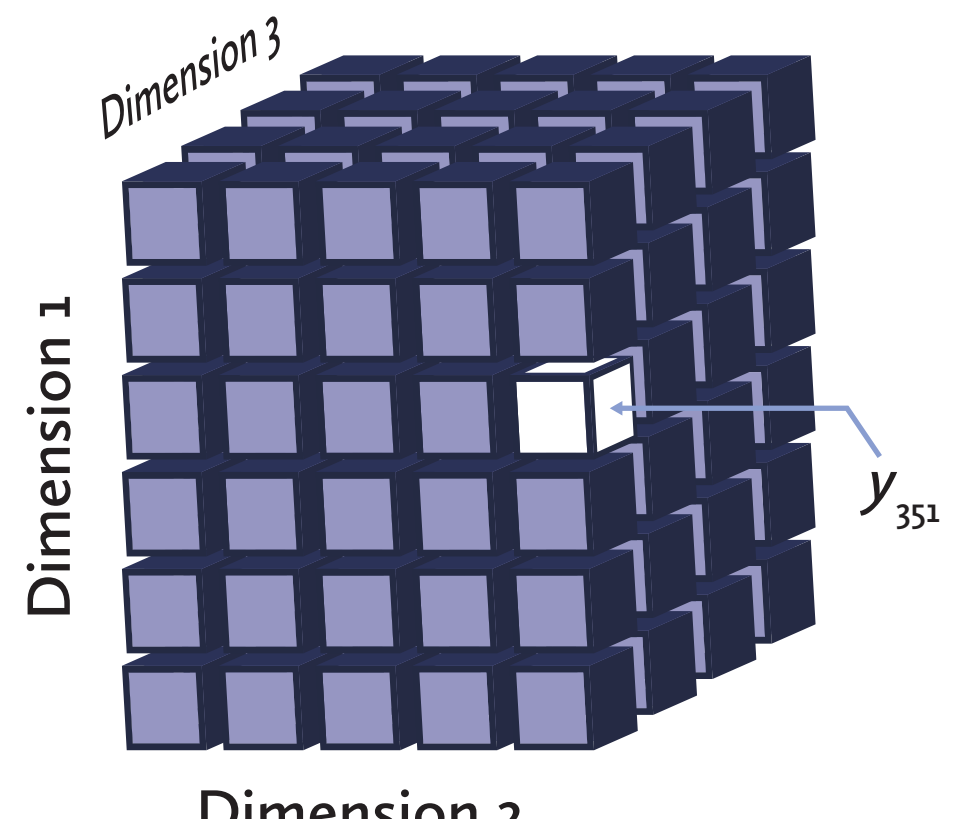

FIGURE 10: A third-order tensor with a highlighted element $y$. 
three-dimensional tensor, we have a non-negative matrix $V$ and we need to calculate non-negative matrix factors $W$ and $H$, such that:

$$
V \approx W H
$$

This means that the product of matrices $W$ and $H$ must be approximately equal to matrix $v$. Matrix $v$ is consisting of $n$ columns which are multivariate data vectors, like the EPSCs of a stimulation train, and $m$ rows which can be different observations, like different synapses. This matrix can be then approximately factorized into an $\mathrm{n} \times \mathrm{r}$ matrix $W$ and an $r \times m$ matrix $H$. Factor $r$ in our case is the TS, LS and recruited components of the EPSC.

In matrix multiplication the dimensions of the factor matrices can be significantly smaller than the dimensions of the product matrix; this is the property that NMF and NTF take advantage of. By trying to keep factor $r$ smaller than $n$ and $m$, the resulting matrices $W$ and $H$ are smaller than $v$. Thus the calculated matrices $W$ and $H$ represent a simplified version of the observed matrix $V$ and can be highly informative as they can uncover hidden structures in the original data.

\section{Gradient descent}

The next step in the method is to determine the quality of the approximation. In essence, the following question must be answered: how well the factorization represents the original data? To answer, the quality of the approximation needs to be quantified. This can be done by a so-called cost function. A cost function is a one-dimensional error measure between the input matrix $V$ and the output product $W H$.

The whole point of the NTF method is to minimize the cost function, thus to decrease as much as possible the error between the measured data and the inferred quantities. We can visualize this process as a landscape where we are situated at the top of a hill; our task is to get to the valley below with as few steps as possible, while blindfolded. Naturally, we will take our first step towards the direction where the slope is the biggest. Then we will decide the direction of our next step again based on the steepest slope. When we are closer 
to the valley, the differences in the slope will be smaller so we have to take small steps towards the steepest slope.

In the previous analogy, the altitude can be translated as the error of the cost function. The goal is to find the smallest value in the cost function and for this the unknown parameter of the model must be tweaked. For the sake of simplification, we will consider that the cost function has only one parameter. The problem is how can we know towards which direction this model parameter must be changed?
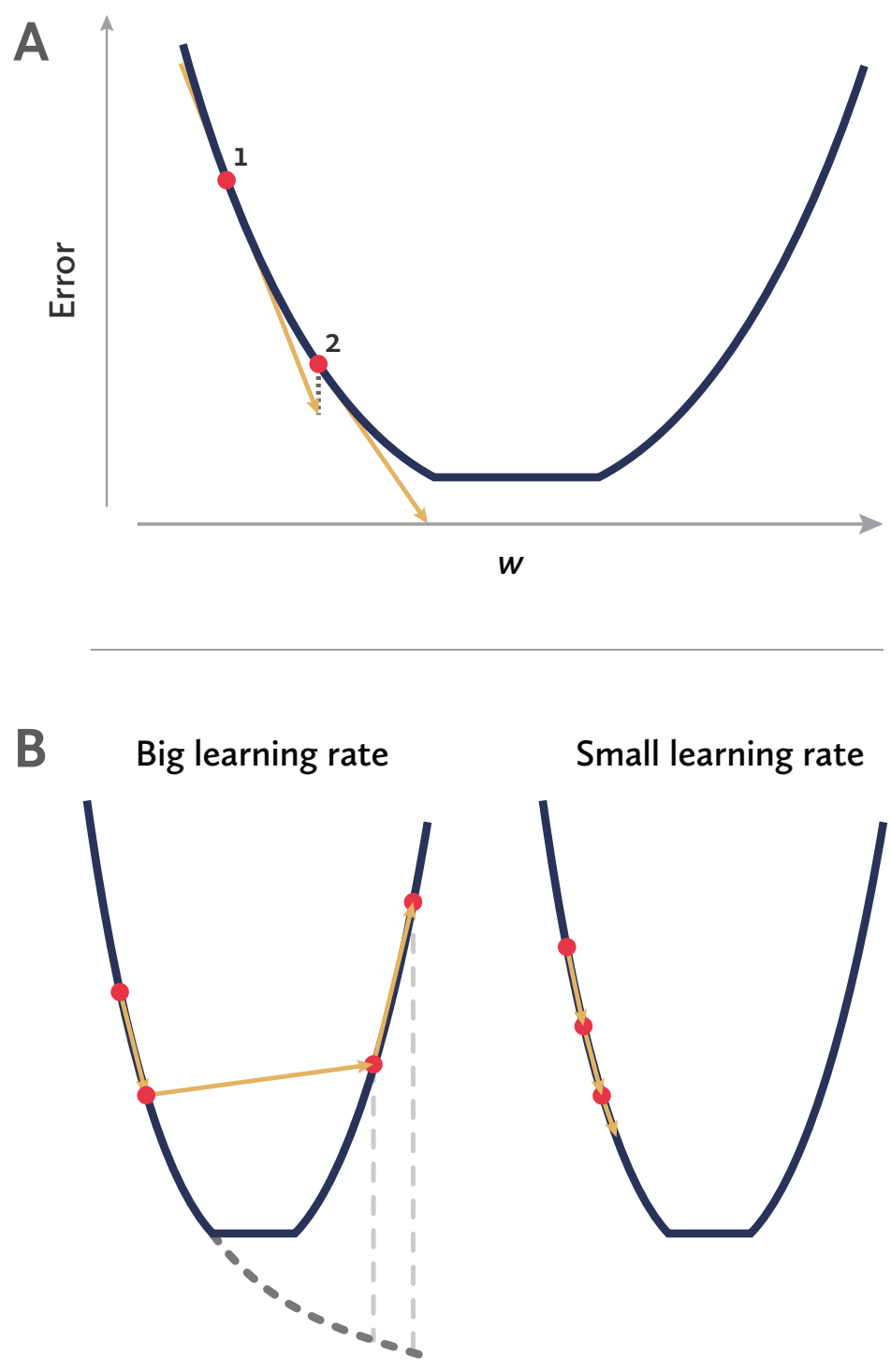

Small learning rate

FIGURE 11: Cost functions and direction of the update rules. A: The derivative of a function with respect to one model parameter results in its slope. B: The importance of the correct learning rate. Left: a big learning rate can result in missing the valley. Right: a small learning rate can take forever to reach the local minima. 
The solution comes from calculus; the derivative of a function with respect to one value is its slope. So, the derivative always points to the direction of the lowest value.

In figure in A, a cost function against one model parameter is plotted. The cost function can be called $C(w)$, where we collectively denote as "model parameter" $w$ the independent variables of the cost function. It is obvious that if the slope for every point $(d C / d w)$ is calculated, then we always get the direction of the local minima or of the lowest error value towards which the model parameter must be shifted to.

If, however, the model parameter is shifted too much, there exists the risk of passing over the valley and thus lose the minima as seen on the left side of figure II B. If it is shifted too little it may take too long to reach the lowest error as seen on the right of figure II B. This property is defined by the so-called step size.

After these parameters have been decided the algorithm needs to take a step towards the direction of the steepest descent i.e. its gradient. This is called an iteration. In every iteration the algorithm moves along the independent variables of the cost function so that the error becomes smaller.

A general update rule for a gradient descent can be written as:

$$
w_{(t+I)}=w_{t}-\eta \nabla C\left(w_{t}\right)
$$

where $t$ is the iteration counter, the minus sign refers to the minimization part of gradient descent, $\eta$ is the step size and $\nabla C\left(w_{t}\right)$ indicates the cost function gradient

$$
\nabla C\left(w_{t}\right)=\left(\begin{array}{c}
\frac{\partial D(w)}{\partial w_{1}} \\
\frac{\partial D(w)}{\partial w_{2}} \\
\vdots \\
\frac{\partial D(w)}{\partial w_{p}}
\end{array}\right)
$$

The writing of this rule can be simplified, by removing the iteration indices: 


$$
w \leftarrow w-\eta \nabla C(w)
$$

However $\eta$ doesn't have to be constant; it can obtain different values for each one of the parameters $w$. In this case the above rule must be rewritten, since the update rules always happen on a weight-byweight basis:

$$
w_{p} \leftarrow w_{p}-\eta_{p} \frac{\partial C(w)}{\partial w_{p}}
$$

In our case, the recorded EPSC amplitudes are probabilistic in their nature; they cannot be precisely predicted since they are subject to quantal noise. This means that there is a certain degree of uncertainty about the precise amount of released neurotransmitter with each action potential (Yarom and Hounsgaard, 20II). Hence, it can be assumed that the amount of neurotransmitter released follows a Poisson distribution. Therefore, the calculated matrixes will represent the maximization of the probability that the originally observed data tensor will be measured, presuming a Poisson distribution of the data.

The cost function and the update rules used in this study were developed by Neher et al. (2009) for decomposing multiply-labeled fluorescence images, based on the algorithms published by Lee and Seung (1999; 2000). Subsequently, they were implemented for use with patch-clamp recordings by Prof. Dr. Dr. h.c. Erwin Neher and kindly provided for using them in my dataset.

The above update rules provide nonnegative representations of the data but cannot guarantee a unique solution. For three-way tensors without the restriction of nonnegativity, uniqueness is certain when the sum of the $k$-ranks of the component matrices is equal or greater than twice the rank of the solution plus 2 (Kruskal, I977). The $k$-rank of a matrix is the maximum number of its columns that are linearly independent. If additional constraints are imposed, such as nonnegativity and sparsity, then uniqueness can be attained more easily (Bruckstein et al., 2007). The sparsity of a matrix refers to the elements of the matrix that are zero. For that reason the starting values of the base functions that will be described in the following paragraphs either start or end up in zero values. 


\section{Starting values}

In order to give the algorithm a head start and to reduce the number of iterations needed in order to reach a satisfactory convergence level, certain starting values for the different weights of the cost function were provided to it: base functions, amplitudes and attenuation factors for all three components. The first iteration is calculated based on these values and then the NTF algorithm proceeds by altering them in order to find the smallest error.

The starting values for the base functions were based on prior models for vesicular release in a two-step fashion (Miki et al., 20I6; Neher and Brose, 20I8). In addition, a basic property of the multiplicative update rules of the NTF was taken advantage of; the estimates of the algorithm cannot cross zero or deviate from it, once they reach zero (Lee and Seung, I999).

The starting values were defined as follows for a factorization to 3 components: TS, LS and RS.

The base function for the Ts component follows a mono-exponential decay:

$$
S_{T S}(y)=e^{-\frac{y}{\tau}}
$$

where $S_{T S}(y)$ is the base function value of the tight-state component at the stimulus number $y$ and $\tau$ is the time constant of decay.

For the base function of the LS component a function was chosen that starts near zero, is gradually augmented and then exponentially decays to zero:

$$
S_{L S}(y)=\left(1-\left(1-S_{L S .0}\right) e^{-\frac{y}{2}}\right) e^{-\frac{y}{4}}
$$

where again $S_{\mathrm{LS}}(y)$ is the value of the Ls base function at stimulus number $y$ and $S_{L S . o}$ is the value of the LS base function at the first stimulation pulse.

Finally, the base function for the newly recruited svs is the following:

$$
S_{r e c}(y)=1-e^{-\frac{y}{4}}
$$


which produces the inverse of an exponential decay.

In figure $12 \mathrm{~A}$ is plotted an example of the starting values for the three base functions.

Next, starting values for the amplitudes $a$ for each component were empirically defined. Ioo svs for the TS, 200 svs for the LS and Io svs for the recruited component were set.

Lastly, attenuation factors $q$ by which the different components are reduced were set, depending on the number of conditioning pulses $n$ that the protocol has. So for the Ts component a factor of $0.6 \times n$ has been set, which is interpreted as a $40 \%$ loss for the Ts component with each conditioning pulse, $0.9 \times n$ for the LS component (Io\% loss for each conditioning pulse) and $0.96 \times n$ for the recruited component (4\% loss).

For reasons that will become clear later on in this chapter, a two-component NTF is sometimes appropriate. Therefore, a distinction is made only between the TS and a combined LS-RS component. If such a factorization is running, then the starting values are as follows:

The base function for the Ts component is the same. However the base function for the combined LS-RS component is the following:

$$
S_{L S, R S}(y)=1-\left(1-S_{L S, R S .0}\right) e^{-\frac{y}{4}}
$$

That produces a curve as seen in fig. I2 B.
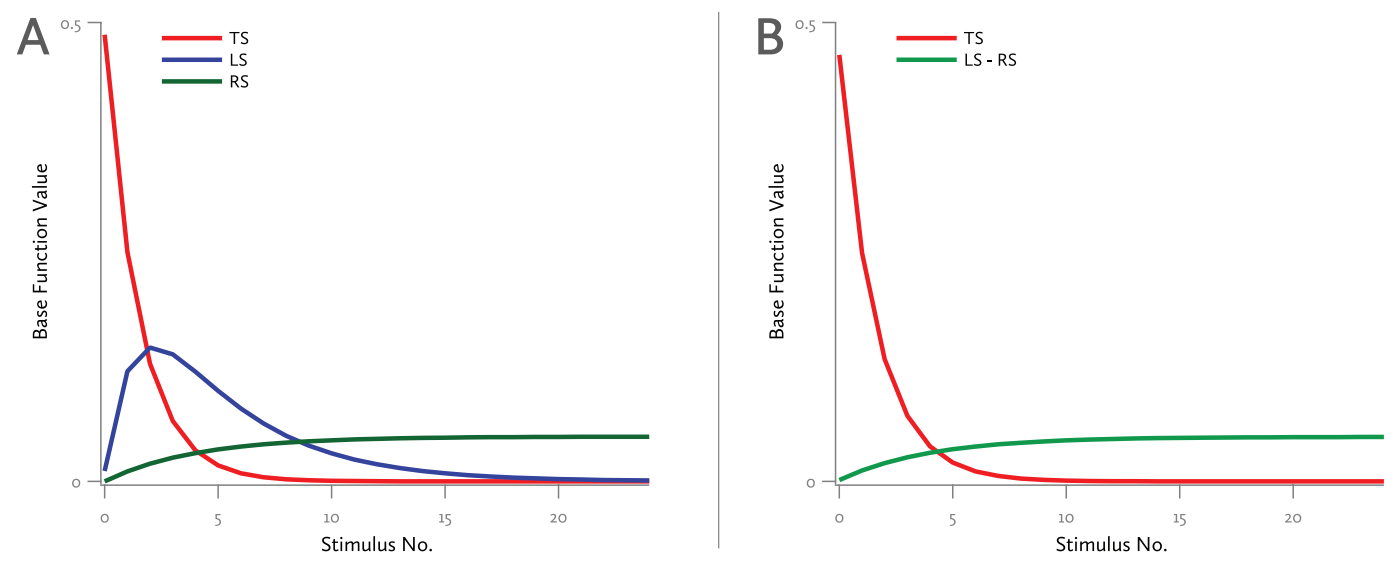

FIGURE 12: An example of the initial values for the base functions. A: Base functions for the decomposition of a mixed signal into three different components. B: Base functions for a two-component decomposition. 
The starting values for the amplitudes are the same, Ioo svs for the TS and 200 SVs for the LS-RS component.

Also, the attenuation factor starting values are similar: 0.6 for the TS and 0.9 for the LS-RS component.

\section{Additional constraints}

Until now references were being made to data that corresponded to stimulation trains of only one frequency and its predepleted variants. So the following question arises: why not inserting a fourth dimension in the NTF so that it can be included in the analysis stimulation trains from different frequencies? This cannot be done because every frequency train's components have different properties. This means that the time course of the LS component in a cell that is stimulated with a $200-\mathrm{Hz}$ train will be quite different than if it's stimulated at $5 \mathrm{~Hz}$. The same holds true for the Ts component's base functions, as well as for the amplitude of each component.

To overcome this problem separate runs for every frequency have been performed; one NTF analysis for the $200-\mathrm{Hz}$ trains and its predepleted variant, another NTF for the Ioo-Hz trains and so on.

This limitation can be used to our advantage, however, by enforcing two additional restrictions during the NTF iterations and therefore limiting the possible solutions of the decomposition process. The first limitation is imposed on the initial value of the Ts component, which is equivalent to the release probability during the first stimulus. sv release during the rested state of the synapse occurs mainly from the Ts synaptic vesicles, since the synapse does not know what frequency of stimuli is going to be used after the first AP. Therefore, the TS components for every frequency train tested should have the same release probability at the first stimulus.

The second restriction occurs in the calculated pool sizes for the TS and LS components for a 3-component NTF. For each cell separately, the calculated pool size for the Ts pool must be as similar as possible for every frequency train tested. The same goes for the LS pool. The logic behind this rule lies in the assumption that svs that are assigned in the TS and LS pools at the beginning of a stimulus train are independent of the stimulus train's frequency. Therefore the total number of svs that are going to be released during a stimu- 
lus train, that were initially either in the TS or in the Ls pool is going to be the same no matter if the stimulation frequency is going to be $200 \mathrm{~Hz}$ or Io $\mathrm{Hz}$, provided that the stimulation train will be long
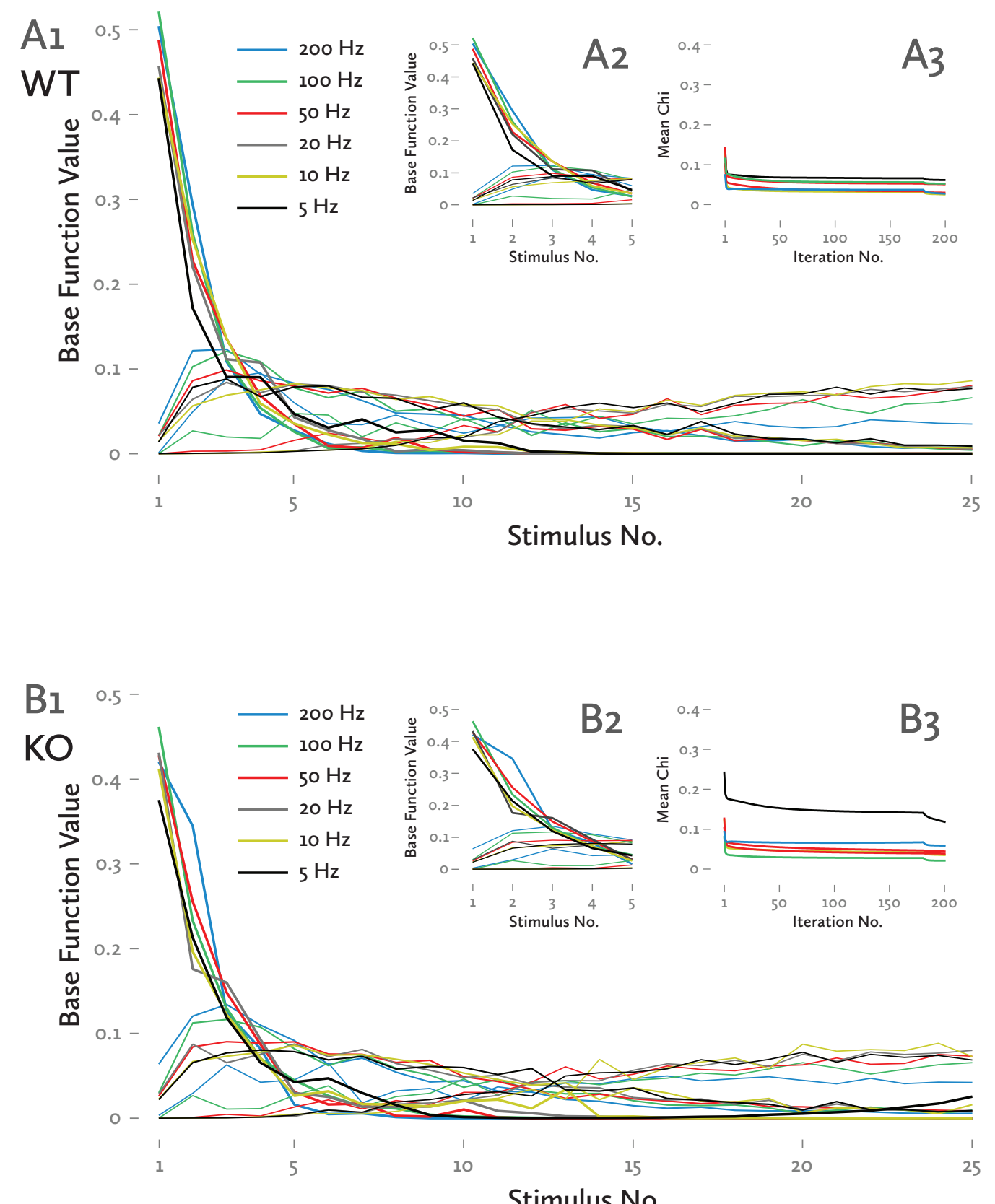

Stimulus No.

FIGURE 13: A 3-component factorization with the same starting values for WT (A) and $K O$ (B) synapse datasets. $A_{1} \& B 1$ : The progression of the base functions for the three components. A2 \& B2: Magnification of the base functions for the 5 first stimuli. $A_{3} \& B_{3}$ : The mean chi-squared values for every iteration of the algorithm. 
enough that both of these pools will get fully depleted. Naturally, for a for a 2-component NTF, this limitation applies only on the TS pool.

\subsubsection{NTF results}

For the NTF analysis, I3 cells in total were used; 6 cells from WT and 7 from Ko animals. Every cell was stimulated with 25-pulse trains at 200, I00, 50, 20, I0 and $5 \mathrm{~Hz}$. Additionally, the synapses were also stimulated with a $200-\mathrm{Hz}$ train preceded by 2 conditioning pulses at Io Hz. The NTF algorithm was run on IGOR Pro (Wavemetrics, USA).

Initially, the dataset was factorized into 3 components, as described previously: a TS, an LS and an RS component. After a trial-and-error process I arrived at a common set of initial values for the WT and KO datasets that produced base-function curves that were quite close to the ones calculated after 200 iterations (fig. I3 AI and BI). The measure of chi-square was similar betweeen WT and ko cells (fig. I3 A3 and $\mathrm{B} 3$ respectively).

A better convergence for the ko dataset was achieved, indicated by an II\% lower chi-square, by increasing the time constant of the Ts initial base function, to 2.4 . In the wT dataset the Ts time constant remained at I.5. The chi-square values are plotted against the iteration number in fig. I4 A3 and B3. All frequencies reached similar convergence levels, except for the $5-\mathrm{Hz}$ stimulation trains at the $\mathrm{KO}$ synapses.

The resulting normalized base functions, showing the progression of the different EPSC components are shown in fig. I4 AI and A2. The $p_{T S . I}$ was estimated for the WT $(0.48 \pm 0.0 \mathrm{I})$ and for the KO calyces (0.34 $\pm 0.0 I$ ) and found to be significantly smaller in the Ko calyces (fig. I4 C) (independent-samples t-test, $t_{(\mathrm{ro})}=8.250, p<.0005$ ).

As mentioned earlier, NTF produces three matrixes. Matrix $A$ is comprised of $a_{c x}$ and it registers each component's amplitude for every cell, in terms of vesicle numbers. This means that an estimate of the three different pools' size for every cell in the dataset can be obtained.

For a formal comparison between WT and kO calyces and for testing for interactions between train frequency and group of cell (WT or kO) on the component's amplitude, a two-way mixed ANOVA was employed. In every comparison that follows in this chapter there 

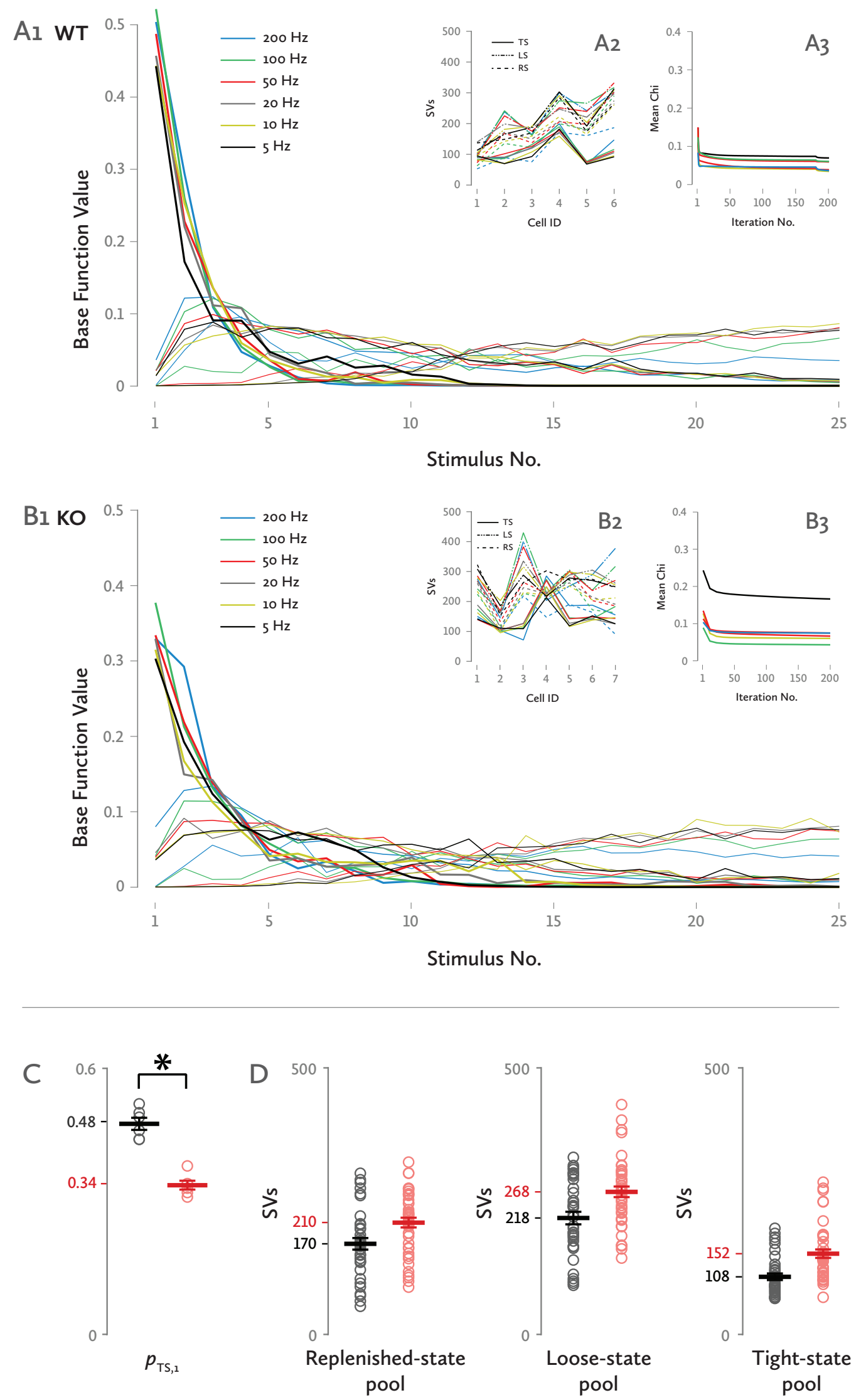
were no outliers, as assessed by examination of studentized residuals for values greater than \pm 3 . The amplitude was normally distributed, as assessed by Normal Q-Q Plot. There was homogeneity of variances, as assessed by Levene's test of homogeneity of variance $(p>.05)$. Mauchly's test of sphericity indicated that the assumption of sphericity was violated for the two-way interaction, therefore the Greenhouse-Geisser estimates were used in all the subsequent comparisons.

The RS component's pool size for every cell can be seen in fig. I4 A2 and B2. There was no statistically significant interaction between frequency and group of cell (WT or KO), $F_{(2.395,26.34 \mathrm{I})}=$ I.I79, $p=.330$, partial $\eta^{2}=.097$. This means that the Rs pool size at every frequency did not depend on whether it was a KO or a WT cell. Also, the RS components' sizes were similar between WT (I70 \pm 22.4 SVs) and KO (209.9 \pm 20.7 sVs) synapses, $F_{(\mathrm{I}, \mathrm{II})}=\mathrm{I} .7 \mathrm{I} 5, p=.2 \mathrm{I} 7$, partial $\eta^{2}=. \mathrm{I} 35$, although slightly higher in the ko dataset. This trend towards a higher Rs pool in the Ko cells that was calculated by the NTF analysis, is also reflected in the slightly elevated replenishment-rate estimation in the ko calyces by using the Schneggenburger et al. (1999) method, as described in chapter 3.3.3.

There was, however, a statistically significant difference between the pool sizes of the RS component at the different frequency trains $F_{(2.395,26.341)}=23.193, p<.0005$, partial $\eta^{2}=.678$. Post-hoc tests showed that the RS component's pool size at the $200-\mathrm{Hz}$ trains was significantly smaller than the pool sizes at all the lower frequencies $(p<$ .oI). The same was also true for the Ioo- $\mathrm{Hz}$ trains $(p<.0005)$. The RS pool sizes at 50-, 20-, I0- and 5- Hz trains did not significantly differ between each other. This refers to the recruitment rate of new svs

FIGURE 14 (OPPOSITE PAGE): A 3-component factorization with optimized starting values, shows that the initial release probability of the TS component is reduced in the KO calyces. Al \& B1: Base function progression during the stimulation trains. Each color represents the NTF analysis for a specific stimulation train frequency. $A_{2} \& B_{2}$ : the sizes of the different SV pools for every cell. $A_{3} \& B_{3}$ : the mean chisquared values for every iteration of the algorithm. C: Estimation of the initial release probability of the TS component. D: Quantification of the three different SV pools. Each cell's pool for every stimulation train frequency is represented with a circle. 
to the LS state during the course of the stimulation train; according to these estimations if the inter-pulse interval is $20 \mathrm{~ms}$ or greater, then the limiting factor for the RS pool's size is the recruitment rate. If, however, the inter-pulse interval is Io ms or shorter, then the RS pool's size becomes smaller, since the rate of release of the svs is greater than the rate of recruitment of new ones.

The next step was to analyze the LS component's pool size. In this pool are those svs that already exist in the loose state in the beginning of a stimulation train and during its course they release their contents. There was no statistically significant interaction between train frequency and group of cell (WT or KO) on the LS pool size, $F_{(2.039,22.425)}=.042, p=.96 \mathrm{I}$, partial $\eta^{2}=.004$. Additionally, the LS component's pool sizes were similar between WT (2I8.4 \pm 26.9 SVs) and KO $\left(267.6 \pm 24.9\right.$ SVs) synapses, $F_{(\mathrm{I}, \mathrm{II})}=\mathrm{I} .803, p=.206$, partial $\eta^{2}=. \mathrm{I} 4 \mathrm{I}$. Finally, there was not a statistically significant effect of the train's frequency on the LS pool size $F_{(2.039,22.425)}=.629, p=.545$, partial $\eta^{2}=.054$, which was expected since the pool size restriction was part of the NTF algorithm constraints.

Lastly, the pool size of the Ts component was examined. The Ts component is comprised of those svs that are in the Ts state in the beginning of a train and are releasing their contents during the course of the stimulations. No statistically significant interaction was found between train frequency and group of cell (WT or KO) on the TS pool size, $F_{(2.514,27.654)}=.180, p=.880$, partial $\eta^{2}=.016$. Moreover, the Ts pool sizes did not statistically differ between WT (I08.2 \pm I8.8 svs) and ко (I5I.5 \pm I7.4 SVs) synapses, $F_{(\mathrm{I}, \mathrm{II})}=2.856, p=. \mathrm{II} 9$, partial $\eta^{2}=.206$, although the ko pool is $40 \%$ larger that the WT one. Finally, there was a statistically significant effect of the train's frequency on the Ts pool size $F_{(2.514,27.654)}=3.497, p=.035$, partial $\eta^{2}=.24 \mathrm{I}$. However, post-hoc tests revealed a statistically significant difference in the Ts pool sizes only between Ioo- $\mathrm{Hz}$ and $\mathrm{IO}-\mathrm{Hz}$ trains $(p=.023)$.

One weakness of the NTF algorithm is that the LS component cannot be properly separated from the $\mathrm{Rs}$; once intracellular $\mathrm{Ca}^{2+}$ starts to rise svs mature from the LS to the Ts state. Simultaneously, new svs are recruited to the LS state, until the LS pool is comprised entirely from newly-recruited svs at some point during the stimulation train. At this point, all of the "original" svs that existed in the Ls pool in the beginning of the train have been released. 
However, the precise time course of these two components is difficult to predict. Therefore, I opted to treat the LS and RS SV pools as one, and factorized my recordings on two components: a TS and a joint LS-RS component.

In order to achieve a tight fit between the initial base functions and the calculated ones, I used the same initial amplitude for the LS component (0.03) and a TS time constant of I.6 for the WT synapses and 2.5 for the Ko ones. I opted for 200 iterations again. The constraint factors were left unchanged: 0.35 amplitude and 0.05 initial release probability for both WT and ko groups (fig. I5).

The differences are clearer; the EPSC $_{\text {TS.I }}$ are more closely converging to $0.43 \pm 0.008$ in the WT synapses and to $0.32 \pm 0.0 \mathrm{I}$ in the kO ones. Thus, the ko calyces have a significantly smaller initial release probability for the Ts component, when compared to the wT calyces (fig. I5 C) (independent-samples t-test, $t_{(\mathrm{Io})}=8.135, p<.0005$ ).

Also, the $200-\mathrm{Hz}$ Ts calculated base function for the ко calyces facilitates in the second pulse, reflecting the paired-pulse facilitation that the Ko synapses have at this frequency (fig. I5 BI).

A closer look at the LS-RS component pool size reveals that there was no statistically significant interaction between frequency and group of cell (WT or KO), $F_{(2.072,22.788)}=.082, p=.927$, partial $\eta^{2}=.007$. Also, the LS-RS component's pool sizes were nearly the same between WT and ko synapses (fig. I5 D) (WT: $368 . \mathrm{I} \pm 45$ sVs, KO: 44I.8 $\pm 4 \mathrm{I} .6 \mathrm{svs}$ ), $F_{(\mathrm{I}, \mathrm{II})}=\mathrm{I} .446, p=.254$, partial $\eta^{2}=. \mathrm{II} 6$. The main effect of frequency indicated a statistically significant difference between the train frequency and the pool size of the LS-RS component $F_{(2.072,22.788)}=5.16 \mathrm{I}$, $p=$. oI3, partial $\eta^{2}=.319$, but post-hoc tests did not reveal any statistically significant differences between the specific frequencies of the stimulation trains.

The calyx-MNTB synapses are likely organized in a structural and functional continuum, where their morphology is correlated with their pool size and short-term plasticity they express. In brief, calyx terminals with relatively simple morphologies display strong shortterm depression after a high-frequency stimulation train. In contrast, calyces with complex morphologies have a larger RRP and exhibit initial facilitation which is followed by short-term depression, when stimulated in the same fashion (Grande and Wang, 20II). The observed cell-to-cell variations in the LS-RS pool sizes (fig. I5 A2 \& 

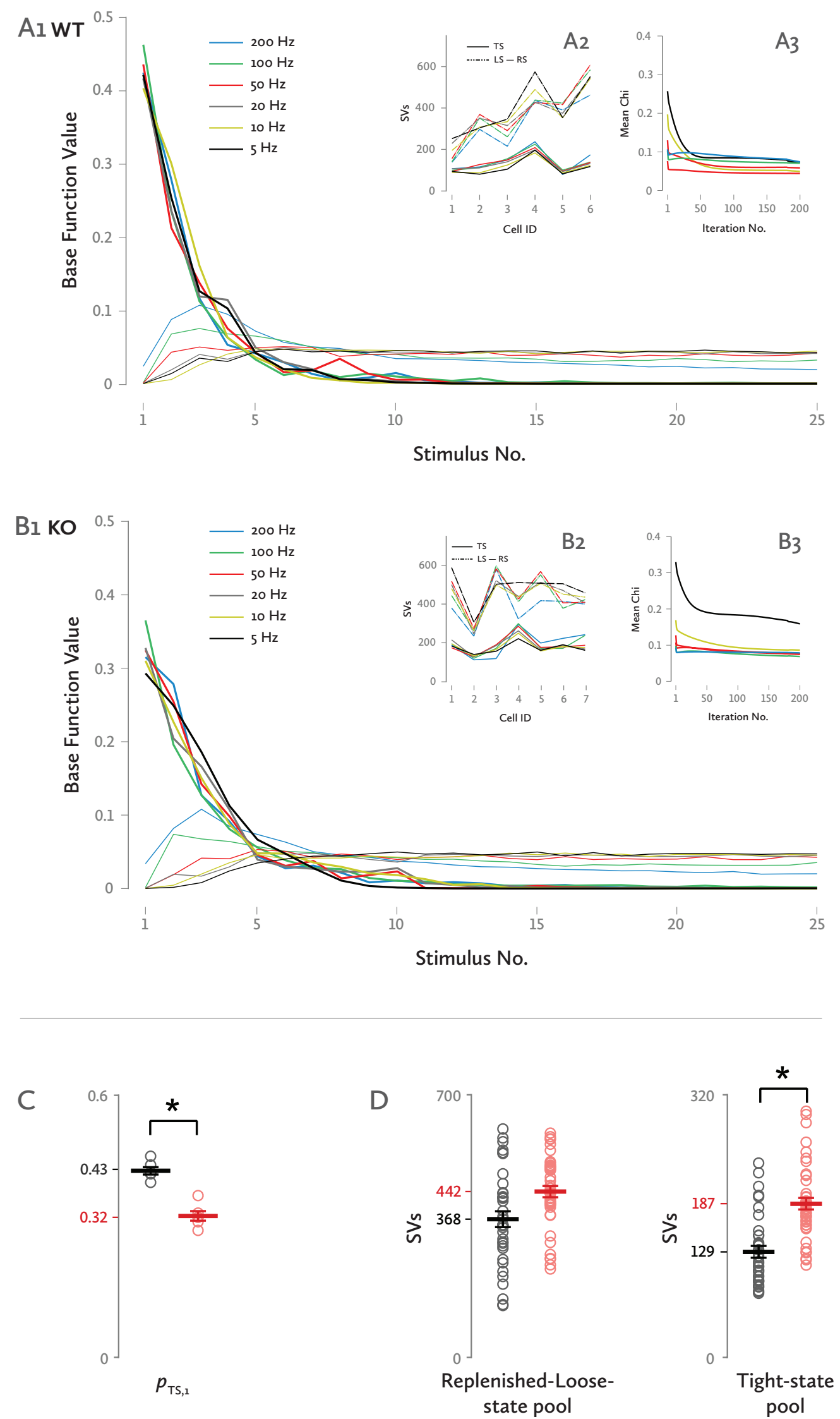

82 
B2) can be explained based on the inherent variability of the calyx of Held synapses (Taschenberger et al., 2016).

The estimation of the Ts component's pool size, for the 2-component analysis, reveals an almost 50\% bigger pool in the kO calyces compared to the WT (fig. I5 D). The KO animals' Ts pool sizes (I87.2 \pm I6.4) were statistically significantly larger compared to the WT ones (I28.5 \pm I7.8 SVs), $F_{(\mathrm{I}, \mathrm{II})}=5.908, p=.033$, partial $\eta^{2}=.349$. This finding points to an altered LS-TS balance; in the Ko calyces the transition rate from LS to TS at rested state could be larger. An alternative explanation could be that the backwards rate, from ts to LS, could be lower.

The bigger Ts pool size in the Ko cells can explain the almost identical $\mathrm{EPSC}_{\mathrm{I}}$ amplitudes seen previously in fig. 3. It can also help explain the slower progression of the train seen in fig. 5 since a bigger pool and a lower release probability can result in more svs being available later on during a train and thus a bigger EPSC amplitude.

In short, the non-negative tensor factorization method provided an approximation of the constitutive components of the EPSC trains. The conclusions reached through this novel method, mainly the reduction of the release probability of a subclass of synaptic vesicles in the ko synapses, corroborate the results observed with the well-established approaches described in the previous chapters.

FIGURE 15 (OPPOSITE PAGE): A 2-component factorization with optimized starting values, again shows that the initial release probability of the TS component is reduced and additionally that the TS component is bigger in the KO calyces. Al \& B1: Base function progression during the stimulation trains. Each color represents the NTF analysis for a specific stimulation train frequency. $A_{2} \& B_{2}$ : the sizes of the different SV pools for every cell. $A_{3} \& B_{3}$ : the mean chi-squared values for every iteration of the algorithm. C: Estimation of the initial release probability of the TS component. D: Quantification of the two different SV pools. Each cell's pool for every stimulation train frequency is represented with a circle. 



\section{Discussion}

\section{I THE PRIMARY FINDING: MOVER IS INCREASING THE INITIAL}

RELEASE PROBABILITY OF TIGHT-STATE SYNAPTIC VESICLES

In this study, I provided evidence that the presynaptic protein Mover acts on a specific subset of docked and primed synaptic vesicles, which is termed "tight state" (TS) according to the terminology introduced by Neher and Brose (2018). More specifically, in the immature calyx of Held of the mouse Mover enhances the initial probability of release of Ts synaptic vesicles. When Mover is knocked-out the size of the Ts pool is significantly increased; consequently, the initial EPSC levels remain unchanged, despite the lower initial release probability of the ко Ts. In addition, the functional predecessors to tight-state svs, the "loose-state" synaptic vesicle pool is unaltered by Mover.

\subsection{THE PREMISE OF THE STUDY: REASONS}

TO STUDY THE ROLE OF MOVER

The need to uncover the functional role of Mover, a largely unstudied vertebrate-specific protein, was the driving force behind this research project. Unlike the ever-evolving postsynapse (Ryan and Grant, 2009), the presynaptic machinery components are mostly evolutionarily conserved; some of the main proteins, like sNAREs, can be traced back to yeast (Kandel, 20I3). Thus, if the presynapse was working just fine, what was the purpose of introducing extra proteins late in the evolutionary game? For this reason, deciphering the functional role of vertebrate-specific proteins like Mover, presents a special interest.

Previous studies on Mover did not reach a satisfactory common ground. Recordings in the calyx of Held of a rat knock-down model showed Mover decreasing the probability of release (Korber et al., 2015). On the other hand, investigating synaptic transmission in the hippocampal mossy fibers of the same mouse Mover knock-out 
model that I used, uncovered an opposite phenotype: higher pairedpulse ratios and higher short-term facilitation on Mover's absence from the presynapse (Viotti, 20I8). Therefore, although the calyx had been studied before in the context of this protein, there were indications that even a change from rat to a mouse model and from a Mover knock down to a knock out is enough to produce markedly different results. Hence, if one was to proceed in studying Mover's influence on synaptic function in the knock-out mouse, the calyx should be the first synapse to be studied.

\subsection{A BRIEF OVERVIEW OF THE RESULTS OF THIS STUDY: DERIVING THE MAJOR CONCLUSION}

Looking at spontaneous transmission in the calyx of Held, no difference in the properties of spontaneous EPSCs was discernible between WT and KO animals. EPSC amplitude, frequency and shape characteristics were unchanged between the two groups. Therefore, it can be concluded that both the basic release machinery and the postsynaptic AMPA receptors were not altered by the absence of Mover.

Stimulating the afferent fibers of the calyx of Held, yielded evoked EPSCs that were similar in WT and Ko. The shape properties of the evoked EPSC, like half width, rise time, decay time constants and charge were the same. However, the ko mice displayed a trend towards smaller EPSC amplitudes. This could indicate a smaller initial probability of release in the Ko synapses.

Using the paired-pulse paradigm to probe further the release properties of the calyx, revealed a consistently larger paired-pulse ratio (PPR) in the KO synapses, at every interstimulus interval tested. This was especially apparent in the interpulse intervals of 5 and Io $\mathrm{ms}$ where the paired-pulse depression of the WT synapses turned into a paired-pulse facilitation in the Ko calyces. An increase in pairedpulse facilitation is usually associated with a reduced release probability (Dobrunz and Stevens, I997; Murthy et al., 1997).

In order to directly calculate the release probability $\left(p_{r}\right)$ of the synapse, I employed a widely-used method first described by Schneggenburger, Meyer and Neher (1999). In short, the EPSCS of a Ioo-Hz stimulus train were cumulatively plotted against the stimulus number. The last data points were fitted with a linear regression line which 
was then back-extrapolated. The point of intersection with the $y$ axis indicated the readily-releasable pool size (RRP) and the slope reflected the rate of $s v$ replenishment. Since, the $p_{r}$ is the fraction of svs released from the RRP, it follows that it can be calculated from the quotient of the initial EPSC amplitude divided by the calculated RRP size. Although the RRP size was not dependent on Mover, the $p_{r}$ was smaller in the Mover Ko calyces.

It should be noted that the $p_{r}$ calculated by this method is the quotient of the $\mathrm{EPSC}_{\mathrm{I}}$ amplitudes over the respective RRP sizes. Although neither the RRP sizes nor the EPSC $_{\mathrm{I}}$ amplitudes differed statistically between wT and KO animals, the $p_{r}$ did differ. This was because the $\mathrm{RRP}$ and the $p_{r}$ were calculated individually for each synapse. Thus, small differences in the numerator $\left(\mathrm{EPSC}_{\mathrm{I}}\right.$ ) and the denominator (RRP) added up and produced $p_{r}$ values that statistically differed between the two experimental groups. Consequently, the cumulative release method offered another indication towards a diminished release probability in Mover Ko animals.

If Mover indeed acts on synaptic transmission by increasing the release probability, how could this finding be integrated with the current views on multiple subpopulations of primed synaptic vesicles? To answer this question, I adopted Neher and Brose's view (2018) as detailed in the introductory section of this dissertation. The authors proposed a two-step priming model where a newly-recruited synaptic vesicle to the active zone must go through an intermediate state before it gets released. This intermediate state is termed "loose state" (LS) and the release can only occur from the "tight state" (TS). "Loose" and "tight" refer to the conformation of the SNARE complex. At rest, synaptic vesicles are in a balance between LS and TS. However, during an increased intra-terminal $\mathrm{Ca}^{2+}$ concentration, such as during a high-frequency stimulation, the transition rate from LS to Ts is enhanced.

The next step was to decompose the EPSCs from the stimulation trains into their constituent components: a TS, an LS and an RS (recruited state). For that purpose a blind decomposition technique was employed, termed non-negative matrix factorization (NTF), which is commonly used to extract hidden information from convoluted datasets (Cichocki, 2009). To run the analysis information was used from six different frequency trains and from a pre-depleted high-fre- 
quency train; all these protocols were applied on every cell included to this dataset.

NTF analysis revealed that the initial release probability of the ко synapses was indeed reduced, but only that of the tight-state component. In contrast, the calculated LS component had a negligible contribution during the first EPSC. Furthermore, the estimation of the various component's pool sizes showed a markedly increased TS pool size in the KO animals, while the LS and the RS components were unchanged.

The reduced release probability and the enhanced pool size of the TS component at the KO animals, can explain the almost similar initial EPSC amplitude; indeed, the TS release probability is reduced by about $38 \%$ at the KO, while the Ts pool size is augmented by $~ 30 \%$. Thus, through an increase in the number of available Ts synaptic vesicles there was a compensation for the fact that a lesser fraction of them would fuse with the active zone membrane at the arrival of an AP at a rested synapse.

However, this sort of compensation could only work for the first EPSC of a stimulation train; differences started to appear at the second EPSC, especially when it would arrive between 5-20 ms after the first one; the PPR was increased at the Ko synapses. Three different processes contribute to the ratio between the first and the second EPSC amplitudes in a stimulus train: depletion of the TS SV pool, rapid conversion of LS vesicles to Ts during the interval between the two pulses and possibly an increase in Ts release probability due to $\mathrm{Ca}^{2+}$-current facilitation or $\mathrm{Ca}^{2+}$ buildup (Neher and Brose, 20I8). Since the Ts initial release probability was decreased in the Ko animals, a larger amount of Ts svs should be released during the second AP. The fact that the paired-pulse depression of the WT calyces turned into a paired-pulse facilitation in the ko ones, can be explained by the contribution of the Ls component which adds up to the neurotransmitter released by the Ts svs.

In a similar way, the EPSC-amplitude progression of a high-frequency train can be explained; if the Ts release probability becomes smaller, more APs will be needed in order for the Ts vesicle pool to be depleted. Indeed the Ts components in the Ko animals take longer to reach zero (fig. I5 BI). This finding corroborates the fact that the EPSC 
I0o-Hz trains of the Ko calyces take longer to reach the same steadystate levels as the WT (fig. 5).

To further corroborate these findings, I employed the superpriming model as it was applied to the calyx of Held by Taschenberger, Woehler and Neher (20I6). Its major difference from the tight-versus-loose-state model is that it assumes neurotransmitter release from both the normal and the superprimed state. Also, svs need not necessarily mature from the normally-primed to the superprimed state, since they can be directly recruited in these states. Employing this model in the present study's data, it was revealed that the ko superprimed synaptic vesicles depress less during a stimulus train, irrespective of the frequency used. Additionally, the superprimed svs had a smaller release probability in the Ko calyces, compared to the WT ones. On the other hand, the normally-primed vesicles had the same narrow range of release probabilities, independently of whether Mover was present or not.

Although the superpriming model is different than the tight-versus-loose-state model, they both divide the fast releasing pool into "reluctant" and "eager-to-be-released" synaptic vesicles. In both models, the "eager" vesicles had a lower release probability when Mover was absent from the synapse, while the "reluctant" svs were unchanged. In this way, the superpriming model provided a useful insight in the world of primed synaptic vesicles and it further supported the conclusions reached through other paths that were described previously in this dissertation.

4.4 METHODOLOGICAL CONSIDERATIONS: POOL

ESTIMATION AND BLIND-SOURCE SEPARATION

The calyx of Held is part of the auditory pathway, the third synapse the auditory information goes through in the central nervous system. The stimulus trains that were used in this research project to probe the synapse's function ranged from 5 to $200 \mathrm{~Hz}$. These stimulation frequencies were well within the range of naturally occurring action potential rates in the immature calyx (Kuner, 2009).

The integration method (Schneggenburger et al., I999) used here to provide a rough estimate of the replenishment rate, RRP size and consequently release probability has been widely used. It relies on 
the assumption that the steady state EPSC amplitudes observed late in a stimulus train are the result of a balance between release and recruitment of new synaptic vesicles. It is also based on the assumptions that svs are recruited with a stable rate throughout the train and that the release probability is also stable during the train. Nevertheless, a formal treatment of this method proves that its estimate is not identical with the pool of vesicles available for release at a rested state; instead it reflects the decrement in SV pool content during a train stimulation. This estimation, though, could be substantially lower than the actual RRP size. Indeed, this is the case when low frequencies are used. However, when the resulting depression is more than $60 \%$, the discrepancy between the integration method's estimation and the true RRP size is small (Neher, 20I5). In the calculations for this research project, Ioo-Hz stimulation trains were used that resulted in steady-state EPSCs that were depressed by about $80 \%$ compared to the initial levels. Assuming that the release probability did not change during the high-frequency train, the release probability estimation provided by the integration method is underestimating the true release probability by around $25 \%$. Thus, the application of the integration method in this dataset can be considered valid within its reasonable limitations.

A final note on the methods used will be on the non-negative tensor factorization (NTF) that was employed to decompose the EPSC trains into their constitutive TS, LS and RS components. The simplest form of NTF is the decomposition of a two-dimensional array of data, hence the name non-negative matrix factorization (NMF). The first implementation of NMF was in image analysis, after Lee and Seung described rules for multiplicative iterations that preserved non-negativity (I999; 2000). Since then, NTF has been used in a wide array of applications from face recognition (Wang et al., 2005) and text mining (Ding et al., 2006) to biological problems like gene expression classification (Brunet et al., 2004; Carmona-Saez et al., 2006) and fluorescence spectroscopy (Gobinet et al., 2004). The NMF algorithm that was used in the present dataset was first developed for use in analysis of multiply-labeled fluorescence images from biological samples (Neher et al., 2009). As such, the use of this decomposition technique on EPSC train data, although for the first time, can be added to a long list of applications. 
One of the few known interaction partners of Mover is the presynaptic protein Bassoon (Kremer et al., 2007). When Bassoon was removed from central excitatory synapses basal synaptic transmission remained unchanged. However, in recordings from the endbulbs of Held the paired-pulse ratio was reduced, while the synapses exhibited a stronger and faster depression. Additionally, the RRP size was reduced and the release probability as measured in the $333-\mathrm{Hz}$ trains, was larger (Mendoza Schulz et al., 20I4). Similarly, in mossy-fiber synapses to cerebellar granule cells short-term depression was enhanced in the absence of Bassoon (Hallermann et al., 20I0). Another effect of Bassoon was in the rate of recovery from depression; both studies concluded that the absence of Bassoon decreases the reloading rate of svs to the RRP. What is striking is the fact that the removal of Bassoon from central glutamatergic synapses produced an almost inverse behavior in terms of synaptic transmission and short-term plasticity, compared to Mover KO at calyces of Held.

Mover and Bassoon not only interact with each other, but also Mover is dependent on Bassoon; when Bassoon is knocked-out from the endbulbs of Held, Mover's expression in these synapses is downregulated (Mendoza Schulz et al., 20I4). Additionally, while Bassoon is in every synapse in vertebrates since it prevents synapse degradation (Okerlund et al., 20I7; Waites et al., 2013), Mover is heterogeneously expressed (Kremer et al., 2007; Wallrafen and Dresbach, 2018). As it was detailed before, Bassoon is reducing release probability while Mover is increasing it. In the absence of Bassoon RRP is decreased, while in the absence of Mover the TS Sv pool is increased. Therefore, a model could be assumed where these two vertebrate-specific presynaptic proteins are acting in balance, influencing the release probability of the synaptic vesicles towards opposite directions. In that way, a highly complicated and multifactorial mechanism like the release probability of synaptic vesicles, could be easily modulated towards higher or lower values depending on the expression of just one protein: Mover. 


\subsection{MOVER'S FUNCTION IN OTHER SYNAPSES OF THE BRAIN}

The calyx of Held is a classic example of a "phasic synapse" meaning that it is depressing after a high-frequency stimulus train. Sometimes, it may exhibit a slight potentiation before the depression. On the other end of the spectrum lie the "phasic synapses" that under continuous stimulation they facilitate, reaching tens or even hundreds of times their initial response levels (Atwood and Karunanithi, 2002).

Typical tonic synapses in the mammalian central nervous system are the ones that hippocampal mossy fibers form with pyramidal CA3 neurons. Under continuous low-frequency stimulation their initially low synaptic response can strongly facilitate (Salin et al., I996). Each mossy-fiber terminal forms a presynaptic bouton with 30 active zones in average and $600-1,200$ synaptic vesicles in what was defined as a putative RRP (Rollenhagen et al., 2007).

The facilitation exhibited by tonic synapses, can be explained by the loose- and tight-state model as follows; during a rested state, the balance between the LS and the Ts pool is shifted towards the loose state. During the stimulus train, there is a rapid $\mathrm{Ca}^{2+}$-dependent conversion from the LS to the tightly-assembled state. In other words, the probability that an $\mathrm{Sv}$ is in the tight conformation is greater during a continuous stimulation. Provided that the conversion of svs into the tight state is greater than the loss of Ts vesicles due to exocytosis, the result is frequency facilitation of the synapse (Neher and Brose, 20I8).

What would have been the results, if - as a thought experiment we were to apply the findings from the calyx of Held Mover Ko experiments to a tonic synapse like the hippocampal mossy fibers? Since the release probability of the Ts vesicles $\left(p_{T S}\right)$ is lower in the Mover KO synapses, but at the same time the Ts pool is larger, we would expect that the first pulse of a train would have been almost identical to that of a wT synapse.

If a second AP would arrive shortly after, it would find the synapse in a state where the transition rate from LS to TS would be increased at the same levels for WT and Ko terminals. However, the original TS pool, comprised of svs that were in the Ts state at rest and did not get released during the first AP, would be bigger in the KO animals thus 
contributing more vesicles during the second pulse. Because of that, the paired-pulse ratio of the ko mossy fibers would be bigger than the WT.

As the train would progress, the large $\mathrm{Ca}^{2+}$ dependency of the LSto-TS conversion, would cause the Ts pool to fill up at the same rate in WT and Ko synapses. The different release probabilities would still exist, since the $p_{T S}$ of the ko vesicles would continue being lower. This would cause the KO synaptic responses to get progressively bigger than the WT ones, as the number of vesicles converted into the tight state would increase. Eventually, a steady state of synaptic responses would be reached since the rate of release of Ts svs would be equal to the rate of vesicle conversion from LS to Ts. This steady state in the Ko synapses would still be bigger than the WT one.

Recordings acquired from the hippocampal mossy fibers of the same Mover knock-out mouse model, follow the pattern described previously. The relationship between the presynaptic afferent fiber activation and the field excitatory postsynaptic potential ( $f$ EPSP) was the same between WT and кo hippocampal slices. This indicated that the synaptic strength of the rested synapse did not change. Also, no changes were registered in miniature postsynaptic currents between the two groups, pointing to an unaltered basal synaptic transmission. (Viotti, 2018)

The paired-pulse ratio showed a small but significant increase in short inter-pulse intervals in the Ko animals, suggesting a reduced release probability. The greatest differences, however, were in short-term plasticity of these synapses. High-frequency stimulation showed a 50\% larger facilitation in the Mover ko mossy fibers than in the wT. Moreover, low-frequency stimulation produced the hallmark attribute of hippocampal mossy fibers, the so-called low-frequency facilitation (Nicoll and Schmitz, 2005). Mover Ko slices facilitated about 40\% more than the WT (Viotti, 20I8). These results corroborate the conclusions in the calyx of Held that show that Mover is a positive regulator of the tight-state synaptic-vesicle release probability.

\subsection{KNOCKDOWN OF MOVER IN RAT VERSUS KNOCKOUT IN THE MOUSE}

The present findings and their interpretation, as they were also extended to the hippocampal mossy fibers, are in sharp contrast to a 
study performed on a Mover knock-down (KD) rat model. The authors tested in vitro synaptic transmission and short-term plasticity at the calyx of Held at postnatal days I2-I3.

While there was no effect on spontaneous release, evoked EPSC amplitude was higher in the KD calyces and PPR at a IO-ms interpulse interval was significantly lower. Accordingly, a 50-pulse Ioo-Hz train showed a faster and fuller depression, while the RRP and the replenishment rate were similar. Presynaptic recordings showed a smaller time constant in the increase of the membrane capacitance in response to a depolarizing pulse, indicating that it required less $\mathrm{Ca}^{2+}$ for the same amount of svs to be released. Based on these results, the authors concluded that Mover in the calyx of Held is decreasing the release probability of synaptic vesicles through a decreased $\mathrm{Ca}^{2+}$ sensitivity of release (Korber et al., 2015).

Although the exact source of the diverging results between that study and the present one is difficult to pinpoint, there are some differences in the experimental procedure which could be important in the present context. A major difference is the approach taken in removing Mover from the synapse; Korber and colleagues chose to inject the globular bushy cells, located in the ventral cochlear nucleus of 2-day old rats with an adeno-associated virus expressing a small hairpin RNA (shRNA) against Mover RNA. Since the axons of the globular bushy cells form the calyces of Held, this manipulation resulted in a $~ 75 \%$ reduction in Mover immunosignal in calyces expressing the KD shRNA. In contrast, our lab used a mouse model where there was a total and whole-body knockout of Mover. The differences are obvious; the KD approach resulted in a targeted but incomplete silencing of the target gene whereas the ko method offered a complete removal, but from every cell of the animal.

A targeted silencing means that only the synapses that are studied have Mover silenced. A system-wide removal of Mover could have unpredictable effects since the synapses that drive globular bushy cells, which in turn give rise to the calyx of Held, also lack Mover and thus may have an altered synaptic activity. This could lead to the activation of a compensatory mechanism in the downstream synaptic pathway. On the other hand, the incomplete silencing of Mover in the knock-down animal model could have undesirable effects also; the remaining Mover molecules could still interact with Bassoon, 
calmodulin or synaptic vesicles, thereby masking or altering the true effect of its complete absence from the synapse.

An often overlooked subject is differences between animal species. Even species closely related as the mouse and the rat (Gibbs et al., 2004), can have striking differences at the central nervous system level. A characteristic example is the cannabinoid receptor differences between these two species. The cannabinoid receptor CBsc is present in the hippocampi of Sprague Dawley (SD) rats and CDI mice but absent from $\mathrm{C} 57 \mathrm{BL} / 6 \mathrm{~J}$ mice, as determined by the inhibition of synaptic glutamate release by the cannabinoid receptor agonist wiN55.2I2-2 (Hoffman et al., 2005). Moreover, the activation or silencing of the cannabinoid receptors had opposite effects on the behavior of the animals, depending on the species. When using the aforementioned agonist in CDI mice their anxiety levels dropped, whereas the same agonist increased anxiety in SD rats. Moreover, the cannabinoid-receptor CBI antagonist AM-25I increased the anxiety levels of CDI mice but reduced them in rats. The synaptic transmission of hippocampal neurons was also markedly different; inhibitory postsynaptic currents in mice were significantly more sensitive to WIN-55.212 than in rats (Haller et al., 2007).

More recently, the lack of an entire class of synapses in mice, as compared to rats, has been shown. Gap junction coupling was absent in the mouse neuroendocrine tuberoinfundibular dopamine (TIDA) neurons, while electrical coupling was prominent in the same TIDA cells of the rat. The result was that the rat TIDA cells displayed robust and stereotyped slow oscillations, while the mice showed a great cellto-cell variability with faster oscillatory patterns (Stagkourakis et al., 2018).

Differences between species have also been observed in the presynaptic machinery. The $\mathrm{Ca}^{2+}$-binding protein calretinin was studied in the calyx of Held of rat and mouse. In Wistar rats calretinin-positive calyces were found to be irregularly intermingled with calretinin-negative synapses throughout the мNтв nucleus, whereas in C57BL / 6 mice calretinin-positive calyces were concentrated in the lateral мNтв. Additionally, calretinin expression levels varied widely between species; at $\mathrm{P} 30 \mathrm{0}$ 75\% of rat calyces contained that protein, but only 30\% of calyces were calretinin-positive in mice (Felmy and Schneggenburger, 2004). This divergence in expression levels could 
affect the $\mathrm{Ca}^{2+}$ sensitivity of the release machinery differently in rats and mice.

Additionally, auditory sensitivity has been studied for various species and specific differences have arisen. It is well documented that although mouse and rat have the same high-frequency sound sensitivity, at around $50 \mathrm{kHz}$, their lower levels vary; in rat the low-frequency limit can reach to $500 \mathrm{~Hz}$ whereas mice can only hear from $\mathrm{I}-2 \mathrm{kHz}$ and higher (Heffner and Masterton, I980; Turner et al., 2005).

Differences in sound perception between species also arise in sound localization. Sound localization refers to an animal's ability to accurately localize a brief sound. The "textbook" versions of the mechanisms involved in mammals are the following; the first mechanism takes advantage of the fact that sounds not arising directly in front or behind the head arrive at different time points at the two ears. The second mechanism is exploiting the "shadowing" effect the head has on sounds with frequencies roughly equal or smaller than its diameter; therefore the sound levels arriving at the two ears are different (Grothe et al., 20Io). Thus, mammals with a big head size should be better at sound localization than others with a smaller head. However this is not the reality; humans, having large interaural distances, display a great localization acuity, but horses and cattle are poorer localizers than most rodents (Heffner and Heffner, I984; Heffner and Heffner, I986)

Most strikingly, even in small rodents head size does not predict sound localization acuity. Although in various species of rats and gerbils sound localization threshold closely follows head size, in grasshopper mice it doesn't; whereas their predicted localization threshold is $53^{\circ}$, in reality it is far lower, at $19^{\circ}$ (Heffner and Heffner, I988). The same holds true for house mice (mus musculus) (Ehret and Dreyer, I984).

The ability of mice to overcome the limitations in sound localization imposed by their relatively small head size, could indicate a functional specialization in the auditory pathway. Incidentally, the calyx of Held synapse is instrumental in sound localization since the MNTB principal cells target the neurons of the lateral superior olive. Both the lateral and the medial superior olive are considered to be the first brain regions where sound localization occurs (Tollin, 2003). 
Considering the previous facts, species differences between mouse and rat should not be ruled out when one is considering the seemingly opposite effects of Mover on neurotransmitter release.

4.8 ADDITIONAL POINTS TO PONDER: LS-TO-TS

TRANSITION VERSUS TS POOL SIZE

The results of this dissertation indicate that the Ts vesicle pool is enlarged in calyces of Held in Mover ko mice. For this enlargement to occur, is Mover affecting the LS-to-Ts transition rate or the number of sv fusion sites? Most of the models agree that the number of fusion sites remain stable during the time span of a stimulus train (Hallermann et al., 20I0; Miki et al., 2016; Zucker, I989).

If the transition rate from LS to TS was increased then this would be manifested in higher time constants for the stimulation trains; more LS svs would be converted in TS vesicles in the same amount of time. Therefore, the Ko synapses would depress slower, since the TS pool would be fastly replenished. Indeed, the experimental observations were on that direction; kO calyces have a larger time constant than the WT ones (Fig. 5). Furthermore, the decomposition provided by the NTF model shows a statistically similar Ls pool size for the two experimental groups, with a slight upwards trend for the ko pool (fig. I4 D).

If, on the other hand, the number of release sites was greater in the Ko animals, and the transition rate was the same as in the WT animals, then we should have witnessed a bigger LS and Ts pool with no apparent change in the EPSC-amplitude dynamics of the stimulation trains. Summing the LS and Ts pools calculated by the 3-component decomposition reveals that the KO calyxes have a $28 \%$ larger vesicle pool, but the difference is non-statistically significant.

Therefore, it remains an open question whether the larger Ts pool size in the Ko animals is due to an increased transition rate from LS to Ts or a larger number of vesicle fusion sites, or both.

\subsection{FUTURE ENDEAVORS}

The present study produced some answers on the question of Mover's influence on synaptic transmission and its effect on synaptic vesicle 
subpools; these results could be further explored through various additional experiments and techniques.

Electron microscopy could provide further insights on the distribution of svs based on their distance from the active zone. Loss of Munci3-family proteins caused a reduction in the number of vesicles within $5 \mathrm{~nm}$ of the active zone and an accumulation at 5-20 $\mathrm{nm}$ (Imig et al., 20I4). Similarly, Syt-I deficient synapses displayed a $35 \%$ reduction in svs closer than $5 \mathrm{~nm}$ to the active zone (Chang et al., 2018). A synapse without Mover may present the opposite picture; NTF analysis showed a $30 \%$ augmentation of the ts vesicle pool in the Ko animals, which could be manifested as an increase in the number of synaptic vesicles within $5 \mathrm{~nm}$ of the active zone of a resting synapse, whereas the vesicle density between 5-20 $\mathrm{nm}$ should be the same between WT and ko active zones. The difference of closely-located svs from the WT animals could become even larger within 5-IO ms after a single AP stimulation, since the depletion of the TS pool will be smaller in the Ko synapses. Accordingly, after a few APs at a high stimulation frequency there should be no differences in Sv distribution between the two experimental groups, as the release will be solely dependent on newly-recruited synaptic vesicles. To observe sv distribution at such a precise timescale, optogenetic stimulation combined with high-pressure freezing on cultured hippocampal synapses would be necessary to be employed.

Altering the external $\mathrm{Ca}^{2+}$ concentration could also be a potential tool which would augment some differences between wT and Mover Ko synapses. Lowering the extracellular $\left[\mathrm{Ca}^{2+}\right]$ from 3 to $\mathrm{I} .5 \mathrm{mM}$ in synapses between presynaptic parallel fibers and postsynaptic molecular layer interneurons in cerebellar brain slices resulted in a reduction of the slow component of the EPSCs during the course of a stimulation train. This was attributed to a decreased $\mathrm{Ca}^{2+}$ entry in the presynapse, since the authors modeled the slow component on a 2-step release which is $\mathrm{Ca}^{2+}$-dependent. Simultaneously, the fast component depressed more slowly during the course of the stimulation train (Miki et al., 20I8)

Trying to extend these findings on the calyx of Held, some interesting predictions can arise. If the external $\left[\mathrm{Ca}^{2+}\right]$ is lowered then the loose-state SV contribution should be accordingly lower throughout the train, owing to the reduced $\mathrm{Ca}^{2+}$-dependent transition rate from 
LS to TS. This change should be of similar magnitude between WT and ko animals. The behavior of the Ts component, though, under reduced extracellular $\left[\mathrm{Ca}^{2+}\right]$ could shine some light on the mechanism by which Mover is acting on the release probability. The Ts component of the WT synapses should depress more slowly than what it did under normal external $\left[\mathrm{Ca}^{2+}\right]$. If the Ts component of the ko calyces, which is already depressing more slowly than its WT counterpart under normal external $\left[\mathrm{Ca}^{2+}\right]$, depresses even more slowly under reduced $\left[\mathrm{Ca}^{2+}\right]$ conditions it could mean that Mover is increasing the $p_{T S}$ independently of the sv $\mathrm{Ca}^{2+}$ sensor. If however the ко Ts component will not show a slower depression than its WT counterpart under reduced external $\left[\mathrm{Ca}^{2+}\right]$, it could indicate that Mover increases the $p_{T S}$ by acting directly on the $\mathrm{Ca}^{2+}$ sensor.

To further elaborate on the mechanism of release probability reduction in the Ko animals, it should be investigated whether it occurs due to a reduced $\mathrm{Ca}^{2+}$ influx in the presynapse or due to a decreased $\mathrm{Ca}^{2+}$ sensitivity. Presynaptic recordings from the calyx of Held can enable us to measure the $\mathrm{Ca}^{2+}$ currents during step depolarizations. Access to the presynapse can also enable the recording of presynaptic action potentials, in order to measure their width in rested and in high-frequency stimulation conditions. If both $\mathrm{Ca}^{2+}$ influx and AP width are the same in WT and Ko synapses, then the observed differences in release probability could be due to an altered sensitivity of the presynaptic machinery to $\mathrm{Ca}^{2+}$ entry. 



\section{References}

Ahmed, S., Wittenmayer, N., Kremer, T., Hoeber, J., Kiran Akula, A., Urlaub, H., Islinger, M., Kirsch, J., Dean, C., and Dresbach, T. (2013). Mover is a homomeric phospho-protein present on synaptic vesicles. PLoS One 8, e63474.

Alabi, A.A., and Tsien, R.W. (2012). Synaptic vesicle pools and dynamics. Cold Spring Harb Perspect Biol 4, aor3680.

Atluri, P.P., and Regehr, W.G. (I996). Determinants of the time course of facilitation at the granule cell to Purkinje cell synapse. J Neurosci $16,566 \mathrm{I}-567 \mathrm{I}$.

Atwood, H.L., and Karunanithi, S. (2002). Diversification of synaptic strength: presynaptic elements. Nat Rev Neurosci 3, 497-516.

Augustin, I., Rosenmund, C., Sudhof, T.C., and Brose, N. (I999). Munci3-I is essential for fusion competence of glutamatergic synaptic vesicles. Nature 400, 457-46I.

Awatramani, G.B., Turecek, R., and Trussell, L.O. (2004). Inhibitory control at a synaptic relay. J Neurosci 24, 2643-2647.

Banks, M.I., and Smith, P.H. (I992). Intracellular recordings from neurobiotin-labeled cells in brain slices of the rat medial nucleus of the trapezoid body. J Neurosci I2, 2819-2837.

Barnes-Davies, M., and Forsythe, I.D. (I995). Pre- and postsynaptic glutamate receptors at a giant excitatory synapse in rat auditory brainstem slices. J Physiol 488 ( Pt 2), 387-406.

Bischofberger, J., Engel, D., Li, L., Geiger, J.R., and Jonas, P. (2006). Patch-clamp recording from mossy fiber terminals in hippocampal slices. Nat Protoc I, 2075-208I.

Bollmann, J.H., Sakmann, B., and Borst, J.G. (2000). Calcium sensitivity of glutamate release in a calyx-type terminal. Science 289 , 953-957.

Borst, J.G., and Sakmann, B. (I996). Calcium influx and transmitter release in a fast CNS synapse. Nature 383, 43I-434.

Borst, J.G., and Sakmann, B. (I998). Facilitation of presynaptic calcium currents in the rat brainstem. J Physiol 513 ( Pt I), I49-I55. 
Brose, N., Petrenko, A.G., Sudhof, T.C., and Jahn, R. (I992). Synaptotagmin: a calcium sensor on the synaptic vesicle surface. Science 256, IO2I-IO25.

Bruckstein, A., Elad, M., and Zibulevsky, M. (2007). A Non-Negative and Sparse Enough Solution of an Underdetermined Linear System of Equations is Unique.

Brunet, J.P., Tamayo, P., Golub, T.R., and Mesirov, J.P. (2004). Metagenes and molecular pattern discovery using matrix factorization. Proc Natl Acad Sci U S A IOI, 4I64-4I69.

Carmona-Saez, P., Pascual-Marqui, R.D., Tirado, F., Carazo, J.M., and Pascual-Montano, A. (2006). Biclustering of gene expression data by Non-smooth Non-negative Matrix Factorization. BMC Bioinformatics 7,78 .

Chang, S., Trimbuch, T., and Rosenmund, C. (2018). Synaptotagmin-I drives synchronous $\mathrm{Ca}(2+)$-triggered fusion by $\mathrm{C}_{2} \mathrm{~B}$-domain-mediated synaptic-vesicle-membrane attachment. Nat Neurosci 2I, 3340.

Chen, G., Harata, N.C., and Tsien, R.W. (2004). Paired-pulse depression of unitary quantal amplitude at single hippocampal synapses. Proc Natl Acad Sci U S A IOI, I063-Io68.

Chuhma, N., and Ohmori, H. (1998). Postnatal development of phaselocked high-fidelity synaptic transmission in the medial nucleus of the trapezoid body of the rat. J Neurosci I8, 512-520.

Cichocki, A. (2009). Nonnegative matrix and tensor factorizations: applications to exploratory multi-way data analysis and blind source separation (Chichester, U.K.: John Wiley).

Cuttle, M.F., Tsujimoto, T., Forsythe, I.D., and Takahashi, T. (I998). Facilitation of the presynaptic calcium current at an auditory synapse in rat brainstem. J Physiol 512 ( Pt 3), 723-729.

de Lange, R.P., de Roos, A.D., and Borst, J.G. (2003). Two modes of vesicle recycling in the rat calyx of Held. J Neurosci 23, IOI64-IoI73. Del Castillo, J., and Katz, B. (I954a). Quantal components of the endplate potential. J Physiol I24, 560-573.

Del Castillo, J., and Katz, B. (I954b). Statistical factors involved in neuromuscular facilitation and depression. J Physiol I24, 574-585.

DeMaria, C.D., Soong, T.W., Alseikhan, B.A., Alvania, R.S., and Yue, D.T. (200I). Calmodulin bifurcates the local $\mathrm{Ca}^{2+}$ signal that modulates $\mathrm{P} / \mathrm{Q}$-type $\mathrm{Ca}^{2+}$ channels. Nature 4II, 484-489. 
Ding, C., Li, T., and Peng, W. (2006). Nonnegative matrix factorization and probabilistic latent semantic indexing: equivalence, chisquare statistic, and a hybrid method. In Proceedings of the 2Ist national conference on Artificial intelligence - Volume I (Boston, Massachusetts: AAAI Press), pp. 342-347.

Dittman, J.S., Kreitzer, A.C., and Regehr, W.G. (2000). Interplay between facilitation, depression, and residual calcium at three presynaptic terminals. J Neurosci 20, I374-I385.

Dobrunz, L.E., and Stevens, C.F. (I997). Heterogeneity of release probability, facilitation, and depletion at central synapses. Neuron I8, 995-I0o8.

Dunlap, K., Luebke, J.l., and Turner, T.J. (I995). Exocytotic $\mathrm{Ca}^{2+}$ channels in mammalian central neurons. Trends Neurosci I8, 89-98.

Eccles, J.C., Katz, B., and Kuffler, S.W. (I94I). Nature of the "endplate potential" in curarized muscle. 4, 362-387.

Ehret, G., and Dreyer, A. (1984). Localization of tones and noise in the horizontal plane by unrestrained house mice (Mus musculus). J Exp Biol I09, I63-I74.

Felmy, F., Neher, E., and Schneggenburger, R. (2003). Probing the intracellular calcium sensitivity of transmitter release during synaptic facilitation. Neuron 37, 80I-8II.

Felmy, F., and Schneggenburger, R. (2004). Developmental expression of the $\mathrm{Ca}^{2+}$-binding proteins calretinin and parvalbumin at the calyx of Held of rats and mice. Eur J Neurosci 20, I473-I482.

Forsythe, I.D., and Barnes-Davies, M. (I993). The binaural auditory pathway: excitatory amino acid receptors mediate dual timecourse excitatory postsynaptic currents in the rat medial nucleus of the trapezoid body. Proc Biol Sci 25I, I5I-I57.

Forsythe, I.D., Tsujimoto, T., Barnes-Davies, M., Cuttle, M.F., and Takahashi, T. (1998). Inactivation of presynaptic calcium current contributes to synaptic depression at a fast central synapse. Neuron $20,797-807$.

Fucile, S., Miledi, R., and Eusebi, F. (2006). Effects of cyclothiazide on GluRi/Ampa receptors. Proc Natl Acad Sci U S A 103, 2943-2947.

Fujita, Y., Shirataki, H., Sakisaka, T., Asakura, T., Ohya, T., Kotani, H., Yokoyama, S., Nishioka, H., Matsuura, Y., et al. (I998). Tomosyn: a syntaxin-I-binding protein that forms a novel complex in the neurotransmitter release process. Neuron 20, 905-915. 
Gasparini, S., Kasyanov, A.M., Pietrobon, D., Voronin, L.L., and Cherubini, E. (200I). Presynaptic R-type calcium channels contribute to fast excitatory synaptic transmission in the rat hippocampus. J Neurosci 2I, 8715-872I.

Geppert, M., Goda, Y., Hammer, R.E., Li, C., Rosahl, T.W., Stevens, C.F., and Sudhof, T.C. (I994). Synaptotagmin I: a major Ca2+ sensor for transmitter release at a central synapse. Cell 79, 717-727.

Gibbs, R.A., Weinstock, G.M., Metzker, M.L., Muzny, D.M., Sodergren, E.J., Scherer, S., Scott, G., Steffen, D., Worley, K.C., Burch, P.E., et al. (2004). Genome sequence of the Brown Norway rat yields insights into mammalian evolution. Nature 428, 493-52I.

Giraudo, C.G., Eng, W.S., Melia, T.J., and Rothman, J.E. (2006). A clamping mechanism involved in SNARE-dependent exocytosis. Science 313, 676-680.

Giraudo, C.G., Garcia-Diaz, A., Eng, W.S., Chen, Y., Hendrickson, W.A., Melia, T.J., and Rothman, J.E. (2009). Alternative zippering as an on-off switch for SNARE-mediated fusion. Science 323, 5I2$5 \mathrm{I} 6$.

Gobinet, C., Perrin, E., and Huez, R. (2004). Application of Non-negative Matrix Factorization to fluorescence spectroscopy. Paper presented at: 2004 I2th European Signal Processing Conference.

Grande, G., and Wang, L.Y. (20II). Morphological and functional continuum underlying heterogeneity in the spiking fidelity at the calyx of Held synapse in vitro. J Neurosci 3I, I3386-I3399.

Grandes, P., and Streit, P. (1989). Glutamate-like immunoreactivity in calyces of Held. J Neurocytol I8, 685-693.

Grothe, B., Pecka, M., and McAlpine, D. (2010). Mechanisms of sound localization in mammals. Physiol Rev 90, 983-IoI2.

Habets, R.L., and Borst, J.G. (2005). Post-tetanic potentiation in the rat calyx of Held synapse. J Physiol 564, I73-187.

Haller, J., Matyas, F., Soproni, K., Varga, B., Barsy, B., Nemeth, B., Mikics, E., Freund, T.F., and Hajos, N. (2007). Correlated species differences in the effects of cannabinoid ligands on anxiety and on GABAergic and glutamatergic synaptic transmission. Eur J Neurosci $25,2445-2456$.

Hallermann, S., Fejtova, A., Schmidt, H., Weyhersmuller, A., Silver, R.A., Gundelfinger, E.D., and Eilers, J. (2010). Bassoon speeds vesicle reloading at a central excitatory synapse. Neuron 68 , 710-723. 
Hammond, C. (20I5). Cellular and molecular neurophysiology, Fourth edition. edn (Amsterdam ; Boston: Elsevier/AP, Academic Press is an imprint of Elsevier).

He, E., Wierda, K., van Westen, R., Broeke, J.H., Toonen, R.F., Cornelisse, L.N., and Verhage, M. (20I7). Munci3-I and Munci8-I together prevent NSF-dependent de-priming of synaptic vesicles. Nat Commun 8, I59I5.

Heffner, H., and Masterton, B. (I980). Hearing in Glires: Domestic rabbit, cotton rat, feral house mouse, and kangaroo rat. 68 , I584I599.

Heffner, H.E., and Heffner, R.S.J.B.n. (1984). Sound localization in large mammals: localization of complex sounds by horses. 98, 54I. Heffner, R., and Heffner, H. (I986). Variation in the use of binaural localization cues among mammals. Paper presented at: Abstr Assoc Res Otolaryngol.

Heffner, R.S., and Heffner, H.E. (I988). Sound localization in a predatory rodent, the northern grasshopper mouse (Onychomys leucogaster). J Comp Psychol 102, 66-7I.

Held, H. (I893). The central auditory system. Arch Anat Physiol Anat Abt I7, 20I-248.

Hippocrates, and Littré, E. (I849). Oeuvres complètes d'Hippocrate, Vol 6, ist edn (Paris: J. B. Bailliere).

Hoffman, A.F., Macgill, A.M., Smith, D., Oz, M., and Lupica, C.R. (2005). Species and strain differences in the expression of a novel glutamate-modulating cannabinoid receptor in the rodent hippocampus. Eur J Neurosci 22, 2387-239I.

$\mathrm{Hsu}, \mathrm{F} ., \mathrm{Hu}, \mathrm{F}$. and Mao, Y. (20I5). Spatiotemporal control of phosphatidylinositol 4-phosphate by Sac2 regulates endocytic recycling. J Cell Biol 209, 97-IIo.

Imig, C., Min, S.W., Krinner, S., Arancillo, M., Rosenmund, C., Sudhof, T.C., Rhee, J., Brose, N., and Cooper, B.H. (20I4). The morphological and molecular nature of synaptic vesicle priming at presynaptic active zones. Neuron 84, 4I6-43I.

Inchauspe, C.G., Martini, F.J., Forsythe, I.D., and Uchitel, O.D. (2004). Functional compensation of $\mathrm{P} / \mathrm{Q}$ by $\mathrm{N}$-type channels blocks shortterm plasticity at the calyx of Held presynaptic terminal. J Neurosci 24, I0379-I0383. 
Ishikawa, T., Sahara, Y., and Takahashi, T. (2002). A single packet of transmitter does not saturate postsynaptic glutamate receptors. Neuron 34, 6I3-62I.

Jahn, R., and Niemann, H. (I994). Molecular mechanisms of clostridial neurotoxins. Ann N Y Acad Sci 733, 245-255.

Jonas, P., Major, G., and Sakmann, B. (I993). Quantal components of unitary EPSCS at the mossy fibre synapse on CA3 pyramidal cells of rat hippocampus. J Physiol 472, 615-663.

Kandel, E.R. (20I3). Principles of neural science, 5 th edn (New York: McGraw-Hill).

Karatekin, E., Di Giovanni, J., Iborra, C., Coleman, J., O'Shaughnessy, B., Seagar, M., and Rothman, J.E. (20I0). A fast, single-vesicle fusion assay mimics physiological SNARE requirements. Proc Natl Acad Sci U S A 107, 3517-3521.

Katz, B., and Miledi, R. (I968). The role of calcium in neuromuscular facilitation. J Physiol 195, 48I-492.

Korber, C., Horstmann, H., Venkataramani, V., Herrmannsdorfer, F., Kremer, T., Kaiser, M., Schwenger, D.B., Ahmed, S., Dean, C., Dresbach, T., et al. (2015). Modulation of Presynaptic Release Probability by the Vertebrate-Specific Protein Mover. Neuron 87, 52I-533.

Kremer, T., Kempf, C., Wittenmayer, N., Nawrotzki, R., Kuner, T., Kirsch, J., and Dresbach, T. (2007). Mover is a novel vertebrate-specific presynaptic protein with differential distribution at subsets of CNS synapses. FEBS Lett 58I, 4727-4733.

Kruskal, J.B. (1977). Three-way arrays: rank and uniqueness of trilinear decompositions, with application to arithmetic complexity and statistics. Linear Algebra and its Applications I8, 95-I38.

Kulesza, R.J., Vinuela, A., Saldana, E., and Berrebi, A.S. (2002). Unbiased stereological estimates of neuron number in subcortical auditory nuclei of the rat. Hear Res $168, \mathrm{I2}-24$.

Kuner, T. (2009). Ultrastructural Organization of Release Sites in the Calyx of Held. In Encyclopedia of Neuroscience, L.R. Squire, ed. (Oxford: Academic Press), pp. 19-24.

Lee, A., Wong, S.T., Gallagher, D., Li, B., Storm, D.R., Scheuer, T., and Catterall, W.A. (I999). $\mathrm{Ca}^{2+} /$ calmodulin binds to and modulates P/Q-type calcium channels. Nature 399, I55-I59. 
Lee, A., Zhou, H., Scheuer, T., and Catterall, W.A. (2003). Molecular determinants of $\mathrm{Ca}(2+)$ /calmodulin-dependent regulation of $\mathrm{Ca}(v) 2 . \mathrm{I}$ channels. Proc Natl Acad Sci U S A I00, I6059-I6064.

Lee, D.D., and Seung, H.S. (I999). Learning the parts of objects by non-negative matrix factorization. Nature 4OI, 788-79I.

Lee, D.D., and Seung, H.S. (200o). Algorithms for non-negative matrix factorization. In Proceedings of the izth International Conference on Neural Information Processing Systems (Denver, CO: MIT Press), pp. 535-54I.

Lee, J.S., Ho, W.K., and Lee, S.H. (2012). Actin-dependent rapid recruitment of reluctant synaptic vesicles into a fast-releasing vesicle pool. Proc Natl Acad Sci U S A 109, E765-774.

Lee, J.S., Ho, W.K., Neher, E., and Lee, S.H. (2013). Superpriming of synaptic vesicles after their recruitment to the readily releasable pool. Proc Natl Acad Sci U S A IIO, I5079-I5084.

Lee, J.S., Kim, M.H., Ho, W.K., and Lee, S.H. (2008). Presynaptic release probability and readily releasable pool size are regulated by two independent mechanisms during posttetanic potentiation at the calyx of Held synapse. J Neurosci 28, 7945-7953.

Li, Z., Burrone, J., Tyler, W.J., Hartman, K.N., Albeanu, D.F., and Murthy, V.N. (2005). Synaptic vesicle recycling studied in transgenic mice expressing synaptopHluorin. Proc Natl Acad Sci U S A I02, 6I3I-6i36.

Lopez-Murcia, F.J., Reim, K., Jahn, O., Taschenberger, H., and Brose, N. (2019). Acute Complexin Knockout Abates Spontaneous and Evoked Transmitter Release. Cell Rep 26, 252I-2530 e2525.

Maximov, A., Tang, J., Yang, X., Pang, Z.P., and Sudhof, T.C. (2009). Complexin controls the force transfer from SNARE complexes to membranes in fusion. Science 323, 516-521.

Meinrenken, C.J., Borst, J.G., and Sakmann, B. (2002). Calcium secretion coupling at calyx of Held governed by nonuniform channel-vesicle topography. J Neurosci 22, I648-1667.

Mendoza Schulz, A., Jing, Z., Sanchez Caro, J.M., Wetzel, F., Dresbach, T., Strenzke, N., Wichmann, C., and Moser, T. (20I4). Bassoon-disruption slows vesicle replenishment and induces homeostatic plasticity at a CNS synapse. EMBO J 33, 5I2-527. 
Meyer, A.C., Neher, E., and Schneggenburger, R. (200I). Estimation of quantal size and number of functional active zones at the calyx of Held synapse by nonstationary EPSC variance analysis. J Neurosci 2I, 7889-7900.

Miki, T., Malagon, G., Pulido, C., Llano, I., Neher, E., and Marty, A. (2016). Actin- and Myosin-Dependent Vesicle Loading of Presynaptic Docking Sites Prior to Exocytosis. Neuron 9I, 808-823.

Miki, T., Nakamura, Y., Malagon, G., Neher, E., and Marty, A. (20I8). Two-component latency distributions indicate two-step vesicular release at simple glutamatergic synapses. Nat Commun 9, 3943.

Mochida, S., Few, A.P., Scheuer, T., and Catterall, W.A. (2008). Regulation of presynaptic $\mathrm{Ca}(\mathrm{V}) 2$.I channels by $\mathrm{Ca}^{2+}$ sensor proteins mediates short-term synaptic plasticity. Neuron 57, 210-216.

Montecucco, C., and Schiavo, G. (I993). Tetanus and botulism neurotoxins: a new group of zinc proteases. Trends Biochem Sci I8, 324-327.

Morest, D.K. (I968). The growth of synaptic endings in the mammalian brain: a study of the calyces of the trapezoid body. Z Anat Entwicklungsgesch I27, 20I-220.

Muller, M., Felmy, F., Schwaller, B., and Schneggenburger, R. (2007). Parvalbumin is a mobile presynaptic $\mathrm{Ca}^{2+}$ buffer in the calyx of Held that accelerates the decay of $\mathrm{Ca}^{2+}$ and short-term facilitation. J Neurosci 27, 226I-227I.

Murthy, V.N., Sejnowski, T.J., and Stevens, C.F. (1997). Heterogeneous Release Properties of Visualized Individual Hippocampal Synapses. Neuron 18 , 599-612.

Murthy, V.N., and Stevens, C.F. (I999). Reversal of synaptic vesicle docking at central synapses. Nat Neurosci 2, 503-507.

Nakamura, T., Yamashita, T., Saitoh, N., and Takahashi, T. (2008). Developmental changes in calcium/calmodulin-dependent inactivation of calcium currents at the rat calyx of Held. J Physiol 586, 2253-226I.

Neher, E. (1998). Usefulness and limitations of linear approximations to the understanding of Ca++ signals. Cell Calcium 24, 345-357.

Neher, E. (2015). Merits and Limitations of Vesicle Pool Models in View of Heterogeneous Populations of Synaptic Vesicles. Neuron 87, II3I-II42. 
Neher, E. (2017). Some Subtle Lessons from the Calyx of Held Synapse. Biophys J II2, 2I5-223.

Neher, E., and Brose, N. (2018). Dynamically Primed Synaptic Vesicle States: Key to Understand Synaptic Short-Term Plasticity. Neuron IOO, I283-I29I.

Neher, E., and Sakaba, T. (200I). Combining deconvolution and noise analysis for the estimation of transmitter release rates at the calyx of held. J Neurosci 2I, 444-46I.

Neher, E., and Sakaba, T. (2008). Multiple roles of calcium ions in the regulation of neurotransmitter release. Neuron 59, 86I-872.

Neher, R.A., Mitkovski, M., Kirchhoff, F., Neher, E., Theis, F.J., and Zeug, A. (2009). Blind source separation techniques for the decomposition of multiply labeled fluorescence images. Biophys J 96, 379I-3800.

Nicoll, R.A., and Schmitz, D. (2005). Synaptic plasticity at hippocampal mossy fibre synapses. Nat Rev Neurosci 6, 863-876.

Okerlund, N.D., Schneider, K., Leal-Ortiz, S., Montenegro-Venegas, C., Kim, S.A., Garner, L.C., Waites, C.L., Gundelfinger, E.D., Reimer, R.J., and Garner, C.C. (20I7). Bassoon Controls Presynaptic Autophagy through Atg5. Neuron 93, 897-9I3 e897.

Olivera, B.M., Miljanich, G.P., Ramachandran, J., and Adams, M.E. (I994). Calcium channel diversity and neurotransmitter release: the omega-conotoxins and omega-agatoxins. Annu Rev Biochem $63,823-867$.

Otis, T., Zhang, S., and Trussell, L.O. (I996). Direct measurement of AMPA receptor desensitization induced by glutamatergic synaptic transmission. J Neurosci I6, 7496-7504.

Partin, K.M., Fleck, M.W., and Mayer, M.L. (1996). AMPA receptor flip/ flop mutants affecting deactivation, desensitization, and modulation by cyclothiazide, aniracetam, and thiocyanate. J Neurosci I6, 6634-6647.

Ramón y Cajal, S. (1909). Histologie du système nerveux de l'homme $\&$ des vertébrés (Paris: Maloine).

Regehr, W.G. (20I2). Short-term presynaptic plasticity. Cold Spring Harb Perspect Biol 4, aoo5702. 
Reim, K., Mansour, M., Varoqueaux, F., McMahon, H.T., Sudhof, T.C., Brose, N., and Rosenmund, C. (200I). Complexins regulate a late step in Ca2+-dependent neurotransmitter release. Cell I04, 7I-8I.

Rizzoli, S.O., and Betz, W.J. (2005). Synaptic vesicle pools. Nat Rev Neurosci 6, 57-69.

Rodriguez-Contreras, A., de Lange, R.P., Lucassen, P.J., and Borst, J.G. (2006). Branching of calyceal afferents during postnatal development in the rat auditory brainstem. J Comp Neurol 496, 2I4-228.

Rollenhagen, A., Satzler, K., Rodriguez, E.P., Jonas, P., Frotscher, M., and Lubke, J.H. (2007). Structural determinants of transmission at large hippocampal mossy fiber synapses. J Neurosci 27, I0434I0444.

Rosenmund, C., and Stevens, C.F. (1996). Definition of the readily releasable pool of vesicles at hippocampal synapses. Neuron I6, II97I207.

Ryan, T.J., and Grant, S.G. (2009). The origin and evolution of synapses. Nat Rev Neurosci IO, 70I-7I2.

Sahara, Y., and Takahashi, T. (200I). Quantal components of the excitatory postsynaptic currents at a rat central auditory synapse. J Physiol 536, I89-I97.

Sakaba, T., and Neher, E. (200I). Calmodulin mediates rapid recruitment of fast-releasing synaptic vesicles at a calyx-type synapse. Neuron 32, III9-II3I.

Salin, P.A., Scanziani, M., Malenka, R.C., and Nicoll, R.A. (I996). Distinct short-term plasticity at two excitatory synapses in the hippocampus. Proc Natl Acad Sci U S A 93, I3304-I3309.

Satzler, K., Sohl, L.F., Bollmann, J.H., Borst, J.G., Frotscher, M., Sakmann, B., and Lubke, J.H. (2002). Three-dimensional reconstruction of a calyx of Held and its postsynaptic principal neuron in the medial nucleus of the trapezoid body. J Neurosci 22, I0567I0579.

Scheuss, V., Schneggenburger, R., and Neher, E. (2002). Separation of presynaptic and postsynaptic contributions to depression by covariance analysis of successive EPSCs at the calyx of Held synapse. J Neurosci 22, 728-739.

Schluter, O.M., Basu, J., Sudhof, T.C., and Rosenmund, C. (2006). Rab3 superprimes synaptic vesicles for release: implications for short-term synaptic plasticity. J Neurosci 26, I239-I246. 
Schneggenburger, R., and Forsythe, I.D. (2006). The calyx of Held. Cell Tissue Res 326, 3II-337.

Schneggenburger, R., Meyer, A.C., and Neher, E. (I999). Released fraction and total size of a pool of immediately available transmitter quanta at a calyx synapse. Neuron 23, 399-409.

Schneggenburger, R., and Neher, E. (2000). Intracellular calcium dependence of transmitter release rates at a fast central synapse. Nature 406, 889-893.

Schneggenburger, R., and Neher, E. (2005). Presynaptic calcium and control of vesicle fusion. Curr Opin Neurobiol 15, 266-274.

Siksou, L., Varoqueaux, F., Pascual, O., Triller, A., Brose, N., and Marty, S. (2009). A common molecular basis for membrane docking and functional priming of synaptic vesicles. Eur J Neurosci 30, 49-56.

Stagkourakis, S., Perez, C.T., Hellysaz, A., Ammari, R., and Broberger, C. (20I8). Network oscillation rules imposed by species-specific electrical coupling. Elife 7.

Stevens, C.F., and Wesseling, J.F. (I999). Identification of a novel process limiting the rate of synaptic vesicle cycling at hippocampal synapses. Neuron 24, I0I7-I028.

Sun, J.Y., and Wu, L.G. (200I). Fast kinetics of exocytosis revealed by simultaneous measurements of presynaptic capacitance and postsynaptic currents at a central synapse. Neuron 30, I7I-I82.

Sun, Y., Olson, R., Horning, M., Armstrong, N., Mayer, M., and Gouaux, E. (2002). Mechanism of glutamate receptor desensitization. Nature 4I7, 245-253.

Takahashi, T. (2005). Dynamic aspects of presynaptic calcium currents mediating synaptic transmission. Cell Calcium 37, 507-5II.

Tang, J., Maximov, A., Shin, O.H., Dai, H., Rizo, J., and Sudhof, T.C. (2006). A complexin/synaptotagmin I switch controls fast synaptic vesicle exocytosis. Cell I26, II75-II87.

Taschenberger, H., Leao, R.M., Rowland, K.C., Spirou, G.A., and von Gersdorff, H. (2002). Optimizing synaptic architecture and efficiency for high-frequency transmission. Neuron 36, II27-II43.

Taschenberger, H., Scheuss, V., and Neher, E. (2005). Release kinetics, quantal parameters and their modulation during short-term depression at a developing synapse in the rat CNS. J Physiol 568, 5I3-537. 
Taschenberger, H., Woehler, A., and Neher, E. (20I6). Superpriming of synaptic vesicles as a common basis for intersynapse variability and modulation of synaptic strength. Proc Natl Acad Sci U S A II3, E4548-4557.

Tollin, D.J. (2003). The lateral superior olive: a functional role in sound source localization. Neuroscientist 9, I27-I43.

Trussell, L.O., Zhang, S., and Raman, I.M. (I993). Desensitization of AMPA receptors upon multiquantal neurotransmitter release. Neuron IO, II85-II96.

Tsujimoto, T., Jeromin, A., Saitoh, N., Roder, J.C., and Takahashi, T. (2002). Neuronal calcium sensor I and activity-dependent facilitation of $\mathrm{P} / \mathrm{Q}-$ type calcium currents at presynaptic nerve terminals. Science 295, 2276-2279.

Turner, J.G., Parrish, J.L., Hughes, L.F., Toth, L.A., and Caspary, D.M. (2005). Hearing in laboratory animals: strain differences and nonauditory effects of noise. Comp Med 55, I2-23.

Varoqueaux, F., Sigler, A., Rhee, J.S., Brose, N., Enk, C., Reim, K., and Rosenmund, C. (2002). Total arrest of spontaneous and evoked synaptic transmission but normal synaptogenesis in the absence of Munci3-mediated vesicle priming. Proc Natl Acad Sci U S A 99, 9037-9042.

Verhage, M., Maia, A.S., Plomp, J.J., Brussaard, A.B., Heeroma, J.H., Vermeer, H., Toonen, R.F., Hammer, R.E., van den Berg, T.K., Missler, M., et al. (2000). Synaptic assembly of the brain in the absence of neurotransmitter secretion. Science 287, 864-869.

Viotti, J.S. (20I8). The presynaptic protein mover buffers synaptic plasticity at the hippocampal mossy fiber synapse.

Voets, T., Toonen, R.F., Brian, E.C., de Wit, H., Moser, T., Rettig, J., Sudhof, T.C., Neher, E., and Verhage, M. (200I). Munci8-I promotes large dense-core vesicle docking. Neuron 3I, 58I-59I.

von Bartheld, C.S., Bahney, J., and Herculano-Houzel, S. (20I6). The search for true numbers of neurons and glial cells in the human brain: A review of 150 years of cell counting. J Comp Neurol 524, 3865-3895.

Waites, C.L., Leal-Ortiz, S.A., Okerlund, N., Dalke, H., Fejtova, A., Altrock, W.D., Gundelfinger, E.D., and Garner, C.C. (2013). Bassoon and Piccolo maintain synapse integrity by regulating protein ubiquitination and degradation. Емво J 32, 954-969. 
Wallrafen, R., and Dresbach, T. (2018). The Presynaptic Protein Mover Is Differentially Expressed Across Brain Areas and Synapse Types. Front Neuroanat I2, 58.

Wang, Y., Jia, Y., Hu, C., and Turk, M. (2005). Non-negative matrix factorization framework for face recognition. 19, 495-5II.

Wang, Z., Liu, H., Gu, Y., and Chapman, E.R. (20II). Reconstituted synaptotagmin I mediates vesicle docking, priming, and fusion. J Cell Biol I95, II59-II70.

Weber, T., Zemelman, B.V., McNew, J.A., Westermann, B., Gmachl, M., Parlati, F., Sollner, T.H., and Rothman, J.E. (I998). SNAREpins: minimal machinery for membrane fusion. Cell 92, 759-772.

Weimer, R.M., Gracheva, E.O., Meyrignac, O., Miller, K.G., Richmond, J.E., and Bessereau, J.L. (2006). UNC-I3 and UNC-Io/rim localize synaptic vesicles to specific membrane domains. J Neurosci 26, 8040-8047.

Wetzel, F., Dresbach, T., Moser, T., and Brose, N. Dynamic expression of mover in rodent endbulbs of held.

Wolfel, M., Lou, X., and Schneggenburger, R. (2007). A mechanism intrinsic to the vesicle fusion machinery determines fast and slow transmitter release at a large CNS synapse. J Neurosci 27, 3198-3210.

Wolfel, M., and Schneggenburger, R. (2003). Presynaptic capacitance measurements and $\mathrm{Ca}^{2+}$ uncaging reveal submillisecond exocytosis kinetics and characterize the $\mathrm{Ca}^{2+}$ sensitivity of vesicle pool depletion at a fast CNS synapse. J Neurosci 23, 7059-7068.

Wong, A.Y., Graham, B.P., Billups, B., and Forsythe, I.D. (2003). Distinguishing between presynaptic and postsynaptic mechanisms of short-term depression during action potential trains. J Neurosci 23, 4868-4877.

Wu, L.G., and Borst, J.G. (I999). The reduced release probability of releasable vesicles during recovery from short-term synaptic depression. Neuron 23, 82I-832.

$\mathrm{Xu}$, J., Mashimo, T., and Sudhof, T.C. (2007). Synaptotagmin-I, -2, and -9: $\mathrm{Ca}(2+)$ sensors for fast release that specify distinct presynaptic properties in subsets of neurons. Neuron 54, 567-58I.

$\mathrm{Xu}, \mathrm{J}$., and $\mathrm{Wu}, \mathrm{L} . \mathrm{G}$. (2005). The decrease in the presynaptic calcium current is a major cause of short-term depression at a calyx-type synapse. Neuron 46, 633-645. 
Yamamoto, Y., Mochida, S., Kurooka, T., and Sakisaka, T. (2009). Reciprocal intramolecular interactions of tomosyn control its inhibitory activity on SNARE complex formation. J Biol Chem 284, I2480I2490.

Yamamoto, Y., Mochida, S., Miyazaki, N., Kawai, K., Fujikura, K., Kurooka, T., Iwasaki, K., and Sakisaka, T. (20I0). Tomosyn inhibits synaptotagmin-I-mediated step of $\mathrm{Ca}^{2+}$-dependent neurotransmitter release through its N-terminal wD40 repeats. J Biol Chem 285, 40943-40955.

Yarom, Y., and Hounsgaard, J. (20II). Voltage fluctuations in neurons: signal or noise? Physiol Rev 9I, 917-929.

Zucker, R.S. (I989). Short-term synaptic plasticity. Annu Rev Neurosci I2, I3-3I.

Zucker, R.S., and Regehr, W.G. (2002). Short-term synaptic plasticity. Annu Rev Physiol 64, 355-405. 


\section{Acknowledgements}

I would not have been able to complete this work without the help and support of many people. In no particular order, I would like to thank the following:

Thomas Dresbach for giving me the opportunity to work on such an exciting topic and for his constant encouragement and support on everything throughout my doctoral studies.

Costas Papatheodoropoulos for his endless hours in teaching electrophysiology, both theoretical and practical.

Erwin Neher for his invaluable help and guidance in navigating the maze of synaptic vesicle priming theories and for providing me the necessary tools to do so.

Camin Dean for the challenge of starting a side project in her lab and for all the fruitful discussions we had.

Tobias Moser for his help in critical moments both before and during my doctoral studies. Also, together with Nils Brose for the useful discussions and suggestions regarding this project.

The late Michael Hörner for introducing me to the neuroscience community of Göttingen.

Captain Fanourakis Foundation for the scholarship supporting my doctoral studies during the academic years 2013-2015.

Also, all the members of the lab for all the discussions we had, as well as the researchers in Camin Dean's lab who always made me feel welcome. In particular, Julio Viotti for the great discussions we had regarding the hippocampus and synaptic plasticity. Additionally, Irmgard Weiss for performing the genotypings and for her immense help in dealing with the bureaucracy of ordering stuff.

Franscisco Lopez for his help on the first steps in setting up patchclamp recordings from MNTB cells in the lab. Oliver Hennig for his unceasing moral support in the course of these years. Donatus Riemann for everything that was almost, but not quite, entirely unlike something helpful.

Christoph Körber for his introduction to brainstem slicing.

Lastly, my family, without whom nothing would have been possible. 